\title{
Transport Motility of Phagosomes on Actin and Microtubules Regulates Timing and Kinetics of Their Maturation
}

\author{
Yanqi Yu ${ }^{\mathrm{a}, 1}$, Zihan Zhang ${ }^{\mathrm{a}, 1}$, Glenn F. W. Walpole ${ }^{\mathrm{b}, \mathrm{c}}$, and Yan Yu $\mathrm{u}^{\mathrm{a}, *}$ \\ aDepartment of Chemistry, Indiana University, Bloomington, IN 47405-7102 \\ bProgram in Cell Biology, The Hospital for Sick Children, Toronto, Ontario, Canada \\ 'Department of Biochemistry, University of Toronto, Ontario, Canada
}

1. Y.-q. Y. and Z. Z. contributed equally to this work

${ }^{*}$ Corresponding Author: Yan Yu, Department of Chemistry, Indiana University, Bloomington, IN 47405-7102, phone 812-855-0593

\section{Email: yy33@iu.edu}

ORCID: Yan Yu (0000-0001-6496-5045), Yanqi Yu (0000-0002-6969-3752)

Author Contributions: Y.-q. Y., Z. Z., and Y. Y. designed research; Y.-q. Y. and Z. Z. performed research and analyzed data; G. F. W. W. contributed critical reagents; Y.-q. Y., Z. Z., and Y. Y. wrote the paper and G. F. W. W. provided critical discussion.

Competing Interest Statement: The authors declare no conflict of interest.

Keywords: Intracellular transport | phagosome maturation | magnetic tweezers | single particle tracking | phagocytosis

\section{This PDF file includes:}

Main Text

Figures 1 to 7 


\begin{abstract}
Phagosome maturation, in which ingested pathogens are degraded through a complex sequence of biochemical changes, is an essential process in infection control and tissue homeostasis. The maturation of phagosomes requires their intracellular transport. However, mechanisms underlying this dynamical-biochemical interdependence is poorly understood due to the lack of methods to independently manipulate either process. Here we present a study to magnetically manipulate the transport of single phagosomes and simultaneously measure their maturation and degradative functions. We reveal that the transport velocity of phagosomes acts as a maturation clock to regulate the timing of biochemical signaling activities in phagosomes. We show that velocity of phagosomes on microtubules determines their rate of fusion with lysosomes and consequently the rate of lumen acidification, following a linear relationship. Meanwhile, the timing of phagosome transport from actin cortex to microtubules controls the kinetics of early phagosome assembly and rate of transition to late phagosomes.
\end{abstract}

\title{
Main Text
}

\section{Introduction}

Phagocytes of the innate immune system engulf and degrade pathogens through a sequence of dynamic biochemical events in phagocytosis. After recognizing ligands on the pathogen, immune cells internalize pathogens into vacuoles called phagosomes and transport them along actin filaments and microtubules from the periphery of the cell to the perinuclear region (Al-Haddad et al., 2001; Araki, 2006; Gibbs, Kitamoto, \& Williams, 2003; Granger, McNee, Allan, \& Woodman, 2014; Harrison \& Grinstein, 2002; Hewage \& Altman, 2018; M. Jiang et al., 2015; Kapitein et al., 2013; Kelleher \& Titus, 1998; Oberhofer et al., 2020; Schroeder, Mitchell, Shuman, Holzbaur, \& Goldman, 2010). In the course of this intracellular transport, phagosomes degrade the pathogens trapped in their lumens through a cascade of biochemical transformations, including the acidification of the lumen (Steinberg, Huynh, \& Grinstein, 2007), the activation of hydrolytic enzymes for pathogen digestion (Lennon-Duménil et al., 2002), and the generation of reactive oxygen species (ROS) (Rybicka, Balce, Chaudhuri, Allan, \& Yates, 2012). This process is known as phagosome maturation. Many pathogens, such as the bacterium Legionella pneumophila, specifically hijack the intracellular trafficking process to alter the degradative function of phagosomes and thus evade immune clearance (Cossart \& Helenius, 2014; Kagan \& Roy, 2002; Smith \& May, 2013). These examples provide evidence that the effective degradation of pathogens relies on the orchestration of the dynamics and biochemical signaling of phagosomes during maturation. However, mechanisms underlying this dynamical-biochemical interdependence are poorly understood.

So far, studies have been mostly limited to establishing the notion that the actin- and microtubulebased transport of phagosomes is required for their fusion with endolysosomes, a key step in maturation. As with endosomes, nascent phagosomes, once formed at the cell periphery, first encounter the actin cortex (Al-Haddad et al., 2001; Araki, 2006; Gibbs et al., 2004; Gibbs et al., 2003; Granger et al., 2014; Harrison \& Grinstein, 2002; Hewage \& Altman, 2018; M. Jiang et al., 2015; Kapitein et al., 2013; Kelleher \& Titus, 1998). During phagosomal biogenesis, nascent phagosomes continuously fuse with endosomes. This fusion is facilitated by proteins including small GTPase Rab5 and phosphatidylinositol 3-monophosphate (PI3P) (Chua \& Deretic, 2004; Duclos et al., 2000; Feliciano, Yoshida, Straight, \& Swanson, 2011; Jeschke \& Haas, 2018; Kitano, Nakaya, Nakamura, Nagata, \& Matsuda, 2008). Phagosome-endosome fusion was found to be attenuated by the disruption of actin or the inhibition of myosin (Arora, Manolson, Downey, Sodek, \& McCulloch, 2000; Gibbs et al., 2003; Guerin \& de Chastellier, 2000). While this indicates that actin-based transport is involved in phagosome-endosome fusion, the exact roles of actin in 
phagosome maturation are largely unknown. After passing through the actin cortex near the cell periphery, phagosomes are then transported in a bi-directional motion along microtubules (Blocker et al., 1997). During this microtubule-based transport process, phagosomes fuse with lysosomes to acquire copies of the vacuolar ATPase (V-ATPase), which drives the acidification of phagosomal lumen (Sun-Wada, Tabata, Kawamura, Aoyama, \& Wada, 2009). As early phagosome markers dissociate from the membrane, phagosomes also acquire Rab7, lysosome-associated membrane glycoproteins (LAMPs) and proteolytic enzymes, to transition into late phagosomes and then phagolysosomes for degradation of luminal contents (Kinchen \& Ravichandran, 2008). It is believed that microtubule-based transport of phagosomes is required for this process, because microtubule depolymerization leads to a significant decrease in phagosome-lysosome fusion (Funato, Beron, Yang, Mukhopadhyay, \& Stahl, 1997), content delivery from late endosomes to phagosomes (Blocker et al., 1996), and acquisition of lysosome markers (Desjardins, Huber, Parton, \& Griffiths, 1994). These conclusions, which were mostly drawn from cytoskeletal disruption experiments, established the important view that the intracellular transport of phagosomes is an integral part of their maturation process. However, findings from those studies do not identify the mechanisms by which the transport of phagosomes regulates the biochemical activities during maturation.

During maturation, phagosomes must continuously interact with endolysosomes and other organelles to complete their biogenesis. The dynamics and subcellular localization of phagosomes and endolysosomes are guided by the polarized tracks of actin filaments and microtubules. Therefore, it is reasonable to hypothesize that the transport of phagosomes on actin filaments and microtubules exerts temporal control over the maturation process by delivering phagosomes and other organelles to the right locations at the right times. Testing this hypothesis, however, poses unique challenges. Each phagosome functions as an independent signaling unit that evolves over time at its own rate. The chemical composition of both its membrane and the contents of its lumen change transiently, as does the dynamics of phagosome transport. Precisely because of such challenges, studies so far have relied heavily on approaches involving pharmacological inhibition to confirm the importance of the phagosome transport without pinpointing the specific mechanisms.

In this study, we overcame these challenges by developing particle sensors as phagocytic targets that allowed us to combine magnetic manipulation, biochemical imaging, and dynamic tracking of single phagosomes in living cells. With this integrated approach, we provided direct evidence that the actin- and microtubule-based transport of phagosomes regulates the timing of the maturation process via distinct mechanisms. Specifically, we show that the velocity of microtubule-dependent transport of phagosomes directly regulates the rate of acidification of their lumen by promoting phagosome-lysosome fusion, but it doesn't influence the final $\mathrm{pH}$ of phagosomes. In contrast, we found that the timing of phagosome transport from the actin cortex to the microtubules controls early phagosome assembly and biogenesis, including the transient recruitment of early phagosome markers (Rab5 and PI3P). This determines the fate and kinetics of the entire phagosome maturation. Taken together, our results elucidate the mechanisms governing the orchestration of the dynamical and biochemical signaling processes that occur during phagosome maturation.

\section{Results}

\section{Design and characterization of $\mathrm{pH}$-responsive rotational particle sensors (RotSensors)}

The decrease the $\mathrm{pH}$ of the lumen of a phagosome is an early indicator of its maturation and a requisite for many subsequent maturation events, including the activation of degradative enzymes and the production of reactive oxygen species (ROS) (Claus et al., 1998; Savina et al., 2006). Therefore, we first designed $\mathrm{pH}$-responsive RotSensors to simultaneously measure both the acidification of single phagosomes and their intracellular transport dynamics, including both translational and rotational movements (Figure 1A). Each RotSensor consists of a pH-responsive 
particle of $1 \mu \mathrm{m}$ in diameter covalently conjugated with a $100 \mathrm{~nm}$ fluorescent particle (Figure 1B). The "snowman"-like design allowed us to track both the translational and rotational motion of single phagosomes encapsulating the RotSensors. The centroid position of individual $1 \mu \mathrm{m}$ particles as a function of time reports the translational motion of single RotSensors. The orientation and projection distance between each pair of particles within a single RotSensor report the azimuthal $(\varphi)$ and polar $(\theta)$ angles, respectively, as previously described (Figure 1B) (Y. Q. Yu, Gao, \& Yu, 2018). To report the $\mathrm{pH}$ changes of the phagosome lumen, the $1 \mu \mathrm{m}$ particle in each RotSensor was biotinylated and subsequently conjugated with two types of streptavidin, one type labeled with the $\mathrm{pH}$-indicator pHrodo Red and the other with the reference dye CF640R. The fluorescence emission of pHrodo Red increases significantly as the $\mathrm{pH}$ of its surroundings decreases from neutral to acidic (Arppe et al., 2014), making it an ideal indicator for phagosomal pH. CF640R was chosen as a reference dye because it is $\mathrm{pH}$ insensitive and photostable (Figure 1-figure supplement 1). We found that streptavidin is critical in the RotSensor design because it acts as a cushion layer to separate the pHrodo Red dye from the particle surface. Without the streptavidin linker, pHrodo Red that was directly conjugated onto the particle surface exhibited little $\mathrm{pH}$ sensitivity.

A

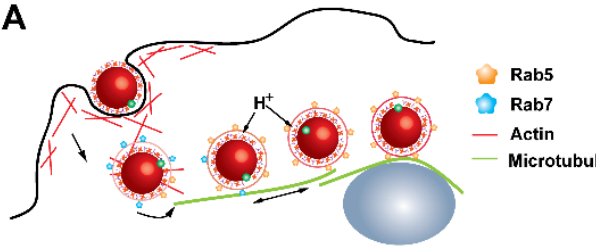

B

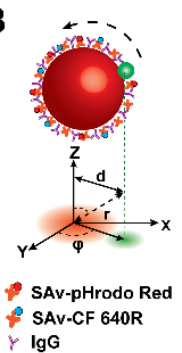

C

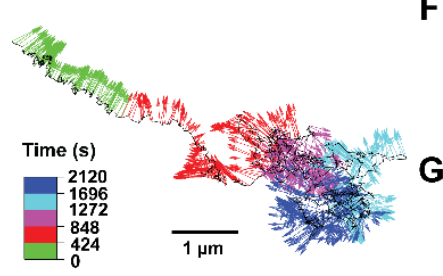

D

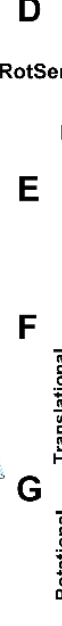

H

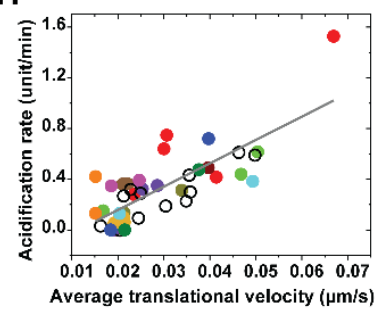

I

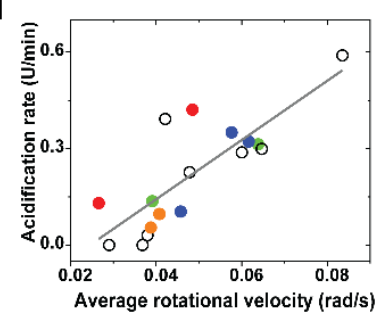

Figure 1. Simultaneous measurement of phagosome transport dynamics and acidification. (A) Schematic illustration of the experimental design. The $\mathrm{pH}$-sensitive rotational particle sensors (RotSensors) were internalized into phagosomes in activated RAW264.7 macrophage cells. (B) Schematic illustration of the design of RotSensors and the principle of single-particle rotational tracking. Each RotSensor contains a $1 \mu \mathrm{m}$ silica particles tethered with a $100 \mathrm{~nm}$ fluorescent particle. The $1 \mu \mathrm{m}$ silica particles were coated with streptavidin (SAv)-pHrodo Red ( $\mathrm{pH}$ reporter dye) and SAv-CF640R (reference dye). The RotSensors were opsonized with physically adsorbed immunoglobulin G. The azimuthal $(\varphi)$ and polar $(\theta)$ angles of each RotSensor were analyzed based on its projection fluorescence image. In the schematic, $d$ indicates the projection inter-particle distance, and $r$ indicates the physical inter-particle distance. (C) The trajectory of a representative RotSensor-containing phagosome in a cell. Black line indicates the translational movement of the centroid of the phagosome. Each vector indicates the orientation of the RotSensor pointing from the centroid of the $1 \mu \mathrm{m}$ particle to that of the $100 \mathrm{~nm}$ particle. Vectors are color-coded based on time. Scale bar, $2 \mu \mathrm{m}$. (D-G) Fluorescence images and line curves showing acidification and transport dynamics of a representative RotSensor-containing phagosome in a cell. In the top row of fluorescence images in $D$, the pHrodoRed-functionalized $1 \mu \mathrm{m}$ particle and its tethered $100 \mathrm{~nm}$ yellow-green particle are shown in red and green, respectively. Fluorescence images in the bottom row in D show the pHrodoRed-functionalized $1 \mu \mathrm{m}$ particle color-coded based on $\mathrm{pH}$. Scale bar, $1 \mu \mathrm{m}$. The phagosome $\mathrm{pH}$ vs. time plot $(\mathbf{E})$ is fitted with sigmoidalBoltzmann function (shown as the black solid line) to determine the initial $\mathrm{pH}$, final $\mathrm{pH}$, the period of rapid acidification $\left(t_{\text {initial }}\right.$ to $\left.t_{\text {final }}\right)$, and acidification rate, as indicated by the red dotted line. (F-G) Plots showing the translational and rotational velocities of the phagosome against time, respectively. Darker lines are velocities values after wavelet denoising. (H-I) Scatter plots showing acidification rates against translational and 
rotational velocities of single phagosomes during rapid acidification period (from $t_{\text {initial }}$ to $t_{\text {final }}$ ). Each data point represents a single phagosome data. Data points from multiple phagosomes within the same cells are shown in the same solid color. Data points from cells containing only one phagosome are shown as black circles. Correlation between the phagosome acidification rate and transport velocities is indicated by the linear regression with a Pearson's coefficient of 0.78 in $(\mathbf{H})$ and 0.81 in (I).

To quantify the $\mathrm{pH}$ response of the RotSensors, we measured the ratio of fluorescence emission intensities of pHrodo Red and of the reference dye CF640R; $I_{p H r o d o} /$ ref, as we varied $\mathrm{pH}$ inside phagosomes in living RAW264.7 macrophage cells and in aqueous buffers (Figure 1-figure supplement 2). For both cases, the ratio $I_{p H r o d o} /$ ref of the RotSensors increased linearly as $\mathrm{pH}$ decreases, which is consistent with previous reports (Deriy et al., 2009). When performing the pH calibration, we observed that the $\mathrm{pH}$ responses of individual RotSensors, including the initial $I_{p H r o d o} / I_{\text {ref }}$ ratio and slope, vary slightly from one to another. This was likely due to the different amounts of dyes on different particles and the different phagosomal lumen environments (Canton, Khezri, Glogauer, \& Grinstein, 2014; Naufer et al., 2018; Nunes, Guido, \& Demaurex, 2015; Ogawa et al., 2010). We compensated for the effect of such variation by performing a normalization analysis in all single phagosome pH measurements (details in Materials and Methods section).

\section{Temporal correlation between different stages of phagosome acidification and transport}

We opsonized pH-sensitive RotSensors with immunoglobulin $\mathrm{G}(\mathrm{IgG})$ to initiate Fc receptor (FcR)dependent phagocytosis in activated RAW264.7 macrophage cells. Using transmission electron microscopy (TEM), we confirmed that $\approx 86 \%$ of internalized RotSensors remained tightly wrapped by the phagosome membrane at the end of our experiments (50 min after the addition of particles to cells) (Figure 1-figure supplement 3). Based on the tight membrane fitting and the presence of IgG binding to FcRs, the RotSensors are unlikely to freely rotate inside the phagosomes and thus their movements faithfully report the movements of the phagosomes they reside in.

We then performed simultaneous three-color live cell imaging of the $\mathrm{pH}$ response and transport dynamics (both translational and rotational) of single phagosomes encapsulating RotSensors. For transport dynamics, we observed that the phagosomes first moved slowly both translationally and rotationally after internalization, but later moved bi-directionally and rapidly with frequent rotations (Figure 1C). During the slow transport period, their average velocities $(0.023 \pm 0.015 \mu \mathrm{m} / \mathrm{s}$ and $0.038 \pm 0.026 \mathrm{rad} / \mathrm{s}$ ) were comparable to that of phagosomes in cells treated with $10 \mu \mathrm{M}$ of the microtubule inhibitor nocodazole $(0.025 \pm 0.015 \mu \mathrm{m} / \mathrm{s}$ and $0.035 \pm 0.023 \mathrm{rad} / \mathrm{s}$; Figure 1 -figure supplement 4). This is evidence that the slow movements are independent of microtubules. We further confirmed that this slow transport period involved movements of phagosomes in the actin cortex, based on the results that the length of the slow transport period changed by Myosin $\mathrm{V}$ and VI inhibition (see details in later section). After a few minutes of slow transport, the phagosomes started rapid bi-directional movements with velocities as high as $0.1 \mu \mathrm{m} / \mathrm{s}$ during runs (representative trajectory in Figure 1C). The phagosomes also rotated frequently in between segments of translational runs with rotational velocities up to $0.15 \mathrm{rad} / \mathrm{s}$. These dynamic behaviors, particularly the phagosome rotation, are characteristic of cargo transport driven by microtubulebased molecular motors, as we previously reported (Gao, Anthony, Yu, Yi, \& Yu, 2018). Such rapid translational and rotational dynamics disappeared in cells treated with nocodazole, which confirms the dependence on microtubules (Figure 1 -figure supplement 5 ). The results show that, during engulfment and the earliest stages of maturation, phagosomes move from the actin cortex to microtubules (schematic illustration in Figure 1A), which agrees with the general notion put forward in previous studies (Al-Haddad et al., 2001; Araki, 2006; Gibbs et al., 2004; Gibbs et al., 2003; 
Granger et al., 2014; Harrison \& Grinstein, 2002; Hewage \& Altman, 2018; M. Jiang et al., 2015; Kapitein et al., 2013; Kelleher \& Titus, 1998).

After engulfment, the phagosomes acidified in their lumen, as indicated by the fluorescence changes of the RotSensors (Figure 1D). By plotting pH vs. time for single phagosomes, we noticed three stages of phagosome acidification. The process started with a standby period during which the phagosome $\mathrm{pH}$ remained at the extracellular $\mathrm{pH}$ of $\approx 7.3$. This was followed by a rapid acidification period when the $\mathrm{pH}$ quickly dropped over a period of a few minutes. Phagosome acidity eventually reached a plateau at $\mathrm{pH} 4.5-5.0$. This acidic $\mathrm{pH}$ is known to be necessary for activating degradative enzymes (Rybicka et al., 2012; Yates, Hermetter, \& Russell, 2005). While individual phagosomes reach slightly different final $\mathrm{pH}$, their $\mathrm{pH}$-time acidification profiles mostly $(\approx 74 \%$ of a total of 57 phagosomes) follow a sigmoidal-Boltzmann function: $p H=p H_{\text {final }}+\frac{p H_{\text {initial }}-p H_{\text {final }}}{1+\exp \left(\frac{t-t_{0}}{d t}\right)}$, in which $t_{0}$ is the half-response point, and $t_{\text {initial }}$ and $t_{\text {final }}$ denote the beginning and the end of the rapid acidification, respectively (Figure $1 \mathrm{E}$ ). Slope at $t_{0}$ is the acidification rate by definition.

We noticed that the beginning of the rapid acidification period appears to coincide with the onset of fast microtubule-based transport (Figure 1E-G). To confirm this observation quantitatively, we imaged phagosome acidification and translational transport in cells expressing actin-GFP, in which intensity changes of actin-GFP can be used to pinpoint the time of particle internalization. As in previous reports (Huang et al., 2014; Scott et al., 2005), we observed that actin rapidly polymerized around nascent phagosomes and then depolymerized at its base, resulting in an actin-GFP intensity peak (Figure 1-figure supplement 6A-B). This actin intensity peak indicates the completion of engulfment (Scott et al., 2005). Using the actin peak as the reference time zero, we found that phagosomes start rapid acidification $620 \pm 292 \mathrm{sec}$ (mean \pm s.d.) after internalization. This time period is referred to as "pH standby time" (Figure 1-figure supplement 6D). Meanwhile, the rapid microtubule-based transport of phagosomes starts $440 \pm 266 \mathrm{sec}$ (mean \pm s.d.) after internalization. During this period, phagosomes move from the actin cortex onto microtubules; it is therefore referred to as the "actin-to-microtubule transition time" (Figure 1-figure supplement 6C). On average, phagosomes start rapid acidification $186 \pm 266 \mathrm{sec}$ (mean \pm s.d.) after they move onto microtubules (Figure 1-figure supplement $6 \mathrm{~F}$ ). When plotting the $\mathrm{pH}$ standby time of single phagosomes against their actin-to-microtubule transition time, we observed that phagosomes with longer actin-to-microtubule transition time on average had longer $\mathrm{pH}$ standby time, as indicated by the linear fitting with a Pearson's coefficient of 0.44 (Figure 1-figure supplement 6E). The results suggest a temporal correlation between the two distinct stages of internalization and transport with phagosome acidification. Phagosomes largely maintain a neutral $\mathrm{pH}$ during their transport within the actin cortex, and rapidly acidify after starting transport on microtubules. We next sought to differentiate the roles of the actin- and microtubule-based transport in phagosome maturation.

\section{Correlation between phagosome acidification kinetics and microtubule-based transport velocity}

We first focused on the stage when phagosomes move on microtubules and also rapidly acidify. We first confirmed that the successful acidification of phagosomes requires intact microtubules, as phagosomes failed to acidify in cells treated with nocodazole (Figure 1-figure supplement 7 ). To search for a possible correlation between the two processes, we calculated the acidification rate of single phagosomes from the sigmoidal fitting of their individual $\mathrm{pH}$-time plots (Figure 1D and Figure 1 -figure supplement 8 ). We then plotted the acidification rate of single phagosomes against their individual translational and rotational velocities during the rapid acidification phase (from $t_{\text {initial }}$ to $\left.t_{\text {final }}\right)$ (Figure $\left.1 \mathrm{H}-\mathrm{I}\right)$. Phagosomes acidify at an average rate of $0.33 \pm 0.28 \mathrm{pH}$ unit $/ \mathrm{min}$, but the acidification rate varies broadly for single phagosomes, even those from the same cells, reflecting the individuality of the phagosome maturation process. Despite this heterogeneity, the acidification 
rates of single phagosomes exhibit a linear relationship with both their translational and rotational velocities with a Pearson's coefficient of 0.78 and 0.81 , respectively. This observation applies not only to phagosomes from different cells but also to those from the same cells. This is shown in Figure $1 \mathrm{H}-\mathrm{I}$, in which data of phagosomes from the same cells are colored the same. It indicates that more motile phagosomes acidify faster. Surprisingly, the final $\mathrm{pH}$ of phagosomes shows no correlation with either the translational or the rotational velocities of the phagosomes (Figure 1figure supplement 7 ). We confirmed that the correlation between phagosome transport dynamics and acidification rate is a general phenomenon independent of the activation status of macrophage cells. Resting RAW264.7 cells, compared to activated cells, have a less flattened shape with fewer visible thick microtubule filaments (Figure 1-figure supplement 9). Phagosomes in resting cells move and acidify slightly faster with less phagosome-to-phagosome variation (Figure 1-figure supplement $10 \mathrm{~A}-\mathrm{B}$ ). Nevertheless, results from both resting and activated cells, when merged, follow a single linear correlation relationship (Figure 1-figure supplement 10A-B), indicating the generality of this observation.

A

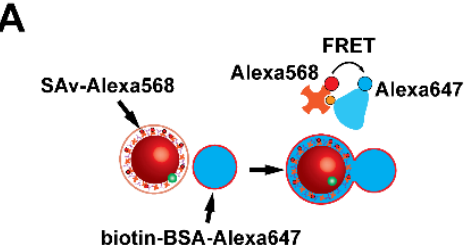

C

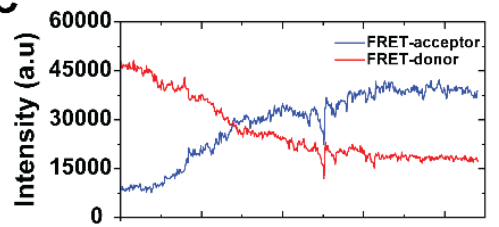

D

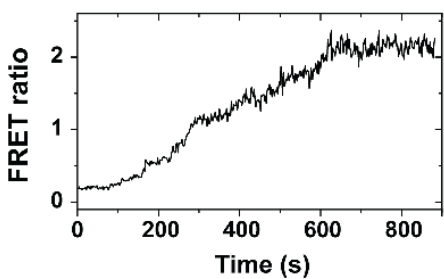

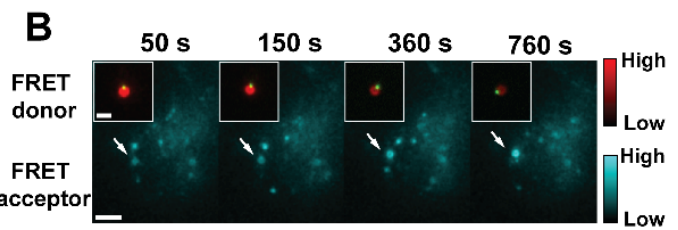

E

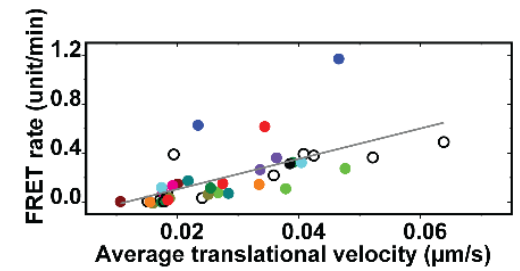

$\mathbf{F}$

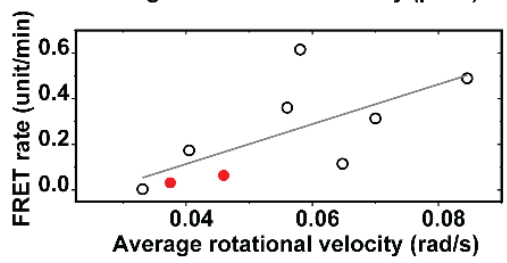

Figure 2. Simultaneous measurement of phagosome-lysosome fusion and phagosome transport dynamics. (A) Schematic illustration of the Förster resonance energy transfer (FRET)-based fusion assay. Streptavidin conjugated with the donor fluorophore Alexa-568 was coated on the RotSensor and BSA-biotin conjugated with the acceptor fluorophore Alexa-647 was encapsulated in lysosomes. Phagosome fusion with lysosomes leads to binding between streptavidin and BSA-biotin, which induces FRET between Alexa-568 and Alexa647. (B-D) Fluorescence images and plots showing the FRET signal change of a representative phagosome during maturation in an activated RAW264.7 macrophage cell. The FRET ratio is defined as the ratio of donor (Alexa568) and acceptor (Alexa647) fluorescence emission intensity under donor excitation. Scale bar, $5 \mu \mathrm{m}$. Scale bar in inset, $2 \mu \mathrm{m}$. (E-F) Scatter plots showing FRET rates against translational and rotational velocities of single phagosomes during phagosome-lysosome fusion period. Each data point represents a single phagosome data. Data points from multiple phagosomes within the same cells are shown in the same solid color. Data points from cells containing only one phagosome are shown as black circles. The black lines indicate linear regression with a Pearson's coefficient of 0.65 in $(\mathbf{E})$ and of 0.68 in $(\mathbf{F})$.

\section{Correlation of phagosome-lysosome fusion kinetics with microtubule-based transport velocity}

During maturation, phagosome acidification is driven by the activity of the proton-pumping vacuolar $\mathrm{H}+-$ ATPase (V-ATPase), which is acquired by fusion with endolysosomes (Canton et al., 2014; Mcneil, Tanasugarn, Meigs, \& Taylor, 1983; Pauwels, Trost, Beyaert, \& Hoffmann, 2017; Riazanski 
et al., 2015; Sun-Wada et al., 2009). Based on our observation that faster moving phagosomes also acidify more rapidly, we next investigated whether phagosome-lysosome fusion also correlates with the motility of phagosomes. We measured the kinetics of phagosome-lysosome fusion using an assay based on Förster Resonance Energy Transfer (FRET). In this assay, FRETsensitive RotSensors were labeled with streptavidin that was conjugated with the donor fluorophore Alexa568 (Figure 2A). Meanwhile, lysosomes were labeled with fluid-phase biotinylated bovine serum albumin (BSA) that was conjugated with the acceptor fluorophore Alexa647. We confirmed that the biotin-BSA-Alexa647 resided in lysosomes based on their colocalization with the lysosome marker LysoTracker in cells (Figure 2-figure supplement 1). The FRET assay worked on the principle that once the contents of lysosomes are delivered to phagosomes during fusion, the biotinBSA-Alexa647 from lysosomes will bind to the streptavidin-Alexa568 on the RotSensors in the phagosomes, leading to increased FRET between Alexa568 and Alexa647. The magnitude of the FRET signal directly indicates the extent of phagosome-lysosome fusion. During live cell imaging, we observed that lysosomes loaded with biotin-BSA-Alexa647, which appeared as fluorescent puncta, docked on phagosomes (Figure 2B). The RotSensors inside phagosomes exhibited decreased emission of Alexa568 (donor) and simultaneous increase of Alexa647 (acceptor) under excitation of the donor fluorophore (a representative phagosome shown in Figure $2 \mathrm{C}$ ). The FRET ratio, defined as the normalized ratio between the donor and acceptor emission intensity, increased continuously until it reached a plateau (Figure 2D). This is a general phenomenon confirmed in over 40 phagosomes from 26 cells (additional representative results are shown in Figure 2-figure supplement 2A). The gradual increase in FRET ratio indicates continuous fusion between the phagosome and labeled lysosomes. The plateau does not necessarily indicate the end of phagosome-lysosome fusion. Instead, it is likely results from all streptavidin binding sites on the RotSensors being occupied by biotin-BSA-Alexa647. Based on the FRET ratio vs. time plots of single phagosomes, we quantified the phagosome-lysosome fusion rate by calculating the slope of the linear portion of each plot (referred to as "FRET rate"). As expected, the FRET rate differed greatly among phagosomes even when they were in the same cell (Figure 2-figure supplement 2). However, all single phagosome FRET ratio data fall into a linear correlation with the translational and rotational velocities of phagosomes, with a Pearson's coefficient of 0.65 and 0.68 , respectively (Figure 2E-F). Surprisingly, phagosomes that move faster along microtubules also tended to fuse more rapidly with lysosomes. Phagosome-lysosome fusion was diminished when microtubules were disrupted by nocodazole treatment, indicating its dependence on microtubules (Figure 2figure supplement 3 ). We next asked whether the faster transport of phagosomes on microtubules promotes their fusion with lysosomes, and hence faster acidification.

\section{Magnetically accelerated phagosome transport promotes acidification via phagosome-lysosome fusion}

To determine whether the motility of phagosomes regulates their acidification and fusion with lysosomes, we applied magnetic tweezers to manipulate the intracellular transport of single phagosomes and simultaneously measure changes in phagosome maturation (Figure 3-figure supplement 1). We designed $1 \mu \mathrm{m}$ magnetically modulated phagosome sensors (MagSensors) that were also $\mathrm{pH}$-responsive (Figure $3 \mathrm{~A}$ ). As for RotSensors, the MagSensors were biotinylated and conjugated with streptavidin-pHrodo Red ( $\mathrm{pH}$ indicator) and streptavidin-CF640R (reference), and exhibited similar $\mathrm{pH}$ responses (Figure 3-figure supplement 2). In all experiments, the solenoid tip was positioned on the opposite cell side from the phagosome of interest to pull it from the cell periphery towards the center. The magnetic force exerted on individual MagSensors changes with their distance from the solenoid tip following the theoretical predictions (Figure 3C) (C. Jiang, Lionberger, Wiener, \& Meyhofer, 2016). On average, the solenoid tip was positioned $\approx 43 \mu \mathrm{m}$ from the MagSensor of interest. This means that the average force on the MagSensors was $\approx 21 \mathrm{pN}$ at 
the beginning of manipulation and reached as high as $\approx 31 \mathrm{pN}$ after they moved closer to the tip at the end of imaging (Figure 3-figure supplement 3).

To ensure that magnetic force was applied only after the internalization of MagSensors, we performed the magnetic experiments in resting actin-GFP-expressing macrophages. We used resting cells for this experiment because they exhibit a similar acidification-phagosome motility correlation to activated cells (Figure 1-figure supplement 10 ) but are less flat which allows for better magnetic manipulation of the phagosome. In these experiments, we turned on the magnetic force immediately after the MagSensors were internalized into phagosomes as indicated by the actin-GFP intensity peak, and pulled the MagSensor-containing phagosomes from the cell periphery towards the center (Figure $3 \mathrm{~A}$ ). The magnetic force remained on throughout imaging. Under the magnetic pulling force, phagosomes moved towards the magnetic tip with accelerated translational velocity (Figure 3-figure supplement 4), but the trajectories of pulled phagosomes were zigzagged (Figure 3B), likely because the magnetic force applied on the MagSensors was only slightly larger than the collective forces from the microtubule-based molecular motors. As mentioned above, the magnetic pulling force on single MagSensors was $\approx 21$ to $31 \mathrm{pN}$ (Figure 3figure supplement 3 ). For comparison, microtubule-based molecular motors were shown to exert collective forces as high as $\approx 20 \mathrm{pN}$ on vacuoles encapsulating $1 \mu \mathrm{m}$ particles (Hendricks, Holzbaur, \& Goldman, 2012). Therefore, the zigzagged movements of phagosomes are plausibly a result of the combined influence of the magnetic force and the forces exerted by molecular motors. As the translational velocity of phagosomes accelerated under magnetic pulling, we found that they also acidified more rapidly. Phagosomes that were magnetically manipulated acidified at an average rate of $0.79 \pm 0.54 \mathrm{pH}$ unit/min $(\mathrm{N}>30)$, in contrast to the average rate of $0.48 \pm 0.34 \mathrm{pH}$ unit $/ \mathrm{min}$ without magnetic forces $(\mathrm{N}>30$ ) (Figure 3E). Surprisingly, the magnetic manipulation had no effects on the final $\mathrm{pH}$ of the phagosomes. Phagosomes with or without magnetic manipulation reached an average final $\mathrm{pH}$ of $4.7 \pm 0.4$ and $4.7 \pm 0.3$, respectively (Figure $3 \mathrm{~F}$ ). This results evidently demonstrate that faster transport of phagosomes leads to their faster acidification.
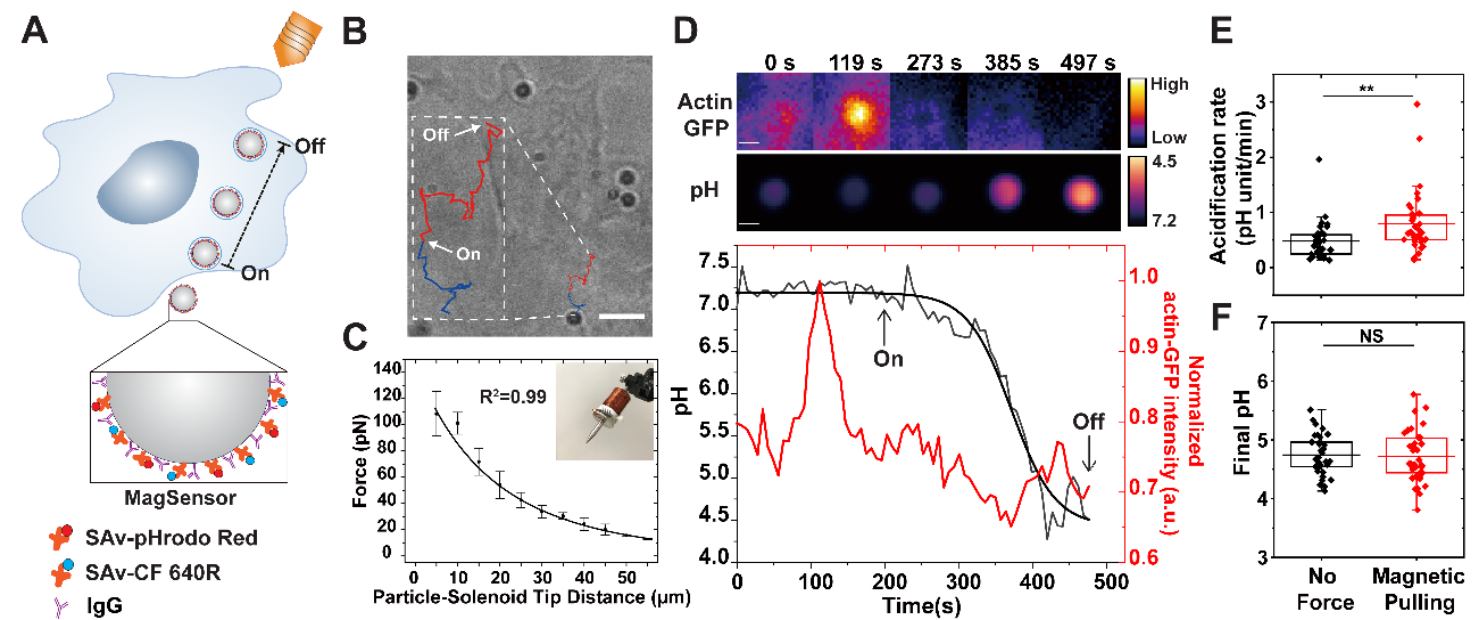

Figure 3. Phagosome acidification during magnetically manipulated transport. (A) Schematic illustration of the experimental design. Magnetic pulling force was applied on MagSensors after internalization in resting RAW 264.7 macrophage cells. The $1 \mu \mathrm{m}$ MagSensors were coated with pHrodo Red-SAv (pH indicator), CF640SAv (reference), and physically adsorbed IgG. (B) Bright-field image of a representative cell overlaid with the trajectory of a MagSensor-containing phagosome under magnetic pulling. The start and end time points of magnetic force are indicated. Scale bar, $5 \mu \mathrm{m}$. (C) Calibration plot showing the magnetic force exerted on the MagSensors as a function of the distance from the MagSensor to the tip of the magnetic tweezers solenoid (shown in inset). Error bars are standard deviation from 5 samples. (D) Fluorescence images and line curves 
showing changes of $\mathrm{pH}$ and actin intensity of a representative phagosome during acidification that was magnetically pulled. Fluorescence images showing the phagosome $\mathrm{pH}$ and actin-GFP in cells are color-coded based on the scales indicated. Scale bars, $1 \mu \mathrm{m}$. The change of phagosome $\mathrm{pH}$ and the corresponding sigmoidal-Boltzmann fitting are plotted in black lines. Data of actin-GFP intensity around the phagosomes are plotted in red lines. The "on" and "off" time points of magnetic pulling are indicated. (E) Statistic result showing the average acidification rate of phagosomes with or without magnetic pulling. The average acidification rate is $0.48 \pm 0.34 \mathrm{pH}$ unit/min without magnetic manipulation $(\mathrm{N}=33)$ and $0.79 \pm 0.54 \mathrm{pH}$ unit/min with magnetic pulling $(\mathrm{N}=38)$. ( $\mathrm{F})$ Statistic result showing the average final $\mathrm{pH}$ in different experiment conditions as indicated. The average final $\mathrm{pH}$ is $4.7 \pm 0.3$ without magnetic manipulation $(\mathrm{N}=33)$ and $4.7 \pm 0.4$ with magnetic pulling $(\mathrm{N}=38$ ). In both scattered plots, each box plot indicates the mean (horizontal line) and the interquartile range from $25 \%$ to $75 \%$ of the corresponding data set. Statistical significance is highlighted by p-values (student's ttest) as follows: ${ }^{* *} p<0.01$, NS $p>0.05$.

We next investigated how magnetic pulling affects phagosome-lysosome fusion using the FRET fusion assay shown in Figure 2. We found that the FRET ratio, which indicates the extent of phagosome-lysosome fusion, increased more rapidly for magnetically pulled phagosomes (representative single phagosome data in Figure 4A). We confirmed the generality of this observation by plotting the results from $>15$ individual phagosomes with and without magnetic forces in heatmaps (Figure 4B). Overall, the FRET signals from magnetically pulled phagosomes were noticeably more intense and increased more rapidly, indicating enhanced phagosome fusion with lysosomes. To investigate whether the movement of phagosomes alone is sufficient to facilitate productive fusion with lysosomes, we next treated cells with nocodazole, which disrupts microtubule-based transport of both phagosomes and lysosomes. We then magnetically moved phagosomes the same way as in non-treated cells. Despite the fast movement of phagosomes under magnetic pulling forces, they exhibited minimal fusion with lysosomes and minimal acidification (Figure 4-figure supplement 1 ). We therefore conclude that productive phagosomelysosome fusion requires active transport of not only the phagosomes but also the lysosomes that they fuse with.

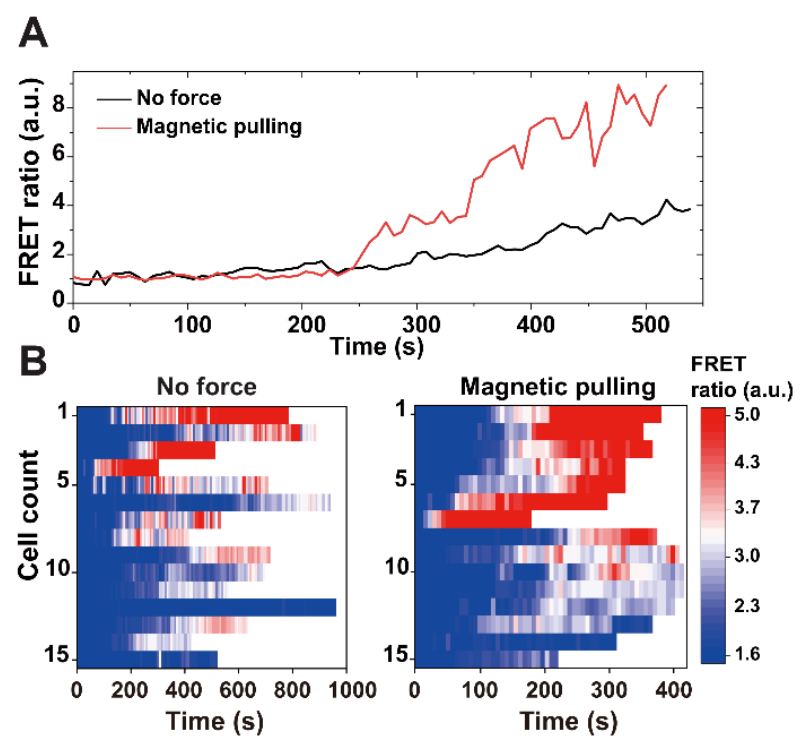

Figure 4. Phagosome-lysosome fusion during magnetically manipulated transport. (A) Line plots showing FRET ratio vs. time for two representative phagosomes with or without magnetic pulling. Time zero indicates the start of magnetic pulling force. (B) Heatmaps showing color-coded FRET ratio vs. time of all phagosomes ( $N=15$ for each graph) with or without magnetic pulling.

The results clearly demonstrate a causal link between microtubule-based transport of phagosomes 
and their maturation. Efficient transport of phagosomes on microtubules promotes their encounters with lysosomes, which causes their acidification. Unexpectedly though, enhancing the movement of engulfed phagocytic targets causes enhanced fusion with lysosomes, and thus, more rapidly acidify. This provides an explanation of why phagosome transport velocity positively correlates with acidification rate and with phagosome-lysosome fusion kinetics (Figure $1 \mathrm{H}-\mathrm{I}$ and Figure 2E-F)

\section{Causal link between actin-based phagosome transport on early phagosome assembly and maturation progression}

We next sought to investigate the role that actin-based phagosome transport plays in the overall process of maturation. Because we observed that phagosomes acidify after transferring onto the microtubules, we expected that the period of phagosome transport in the actin cortex should overlap with early maturation activities prior to acidification. The first maturation activity we measured was the transient recruitment of the small GTPase Rab5 and its subsequent replacement by Rab7. Rab5 and Rab7 are markers for early and late phagosomes, respectively (Poteryaev, Datta, Ackema, Zerial, \& Spang, 2010; Rink, Ghigo, Kalaidzidis, \& Zerial, 2005). The Rab5-to-Rab7 transition is a key mechanism of the progression of phagosome maturation (Kinchen \& Ravichandran, 2008; Mottola, 2014; Poteryaev et al., 2010), so we simultaneously monitored both by imaging Rab5-RFP with actin-GFP or Rab7-GFP in cells. We began by confirming that Rab5 was rapidly recruited to phagosomal membrane after particle engulfment, as indicated by the actinGFP intensity peak (Figure 5-figure supplement 1). After its transient accumulation, Rab5 intensity gradually decreased over time with large fluctuations. Meanwhile, Rab7 was recruited to the same phagosomes until its intensity reached a plateau (Figure 5A-B). This Rab5-to-Rab7 transition was observed in most phagosomes, but the intensity and duration of Rab5 association varied significantly among different phagosomes (Figure 5-figure supplement 2A-B). While Rab5 quickly dissociated within a few minutes on some phagosomes, it remained for a prolonged period on others (Figure 5-figure supplement 3). We then imaged the recruitment of each GTPase in tandem with phagosome acidification (Figure 5C-D). It is clear that the phagosomes remain at a neutral pH when a transiently high level of Rab5 is present on the phagosome membrane. As Rab5 dissociates from phagosomes and Rab7 is recruited, phagosomes start to acidify. The sequence of events is schematically illustrated in Figure 5E. By plotting the recruitment of Rab5 and Rab7 together with the translational velocity of phagosomes as a function of time, we observed that Rab5 recruitment starts during the slow transport of phagosomes within the actin cortex, but its replacement by Rab7 can continue into the period when phagosomes are transported along microtubules (Figure 5figure supplement $2 \mathrm{C}$ ).
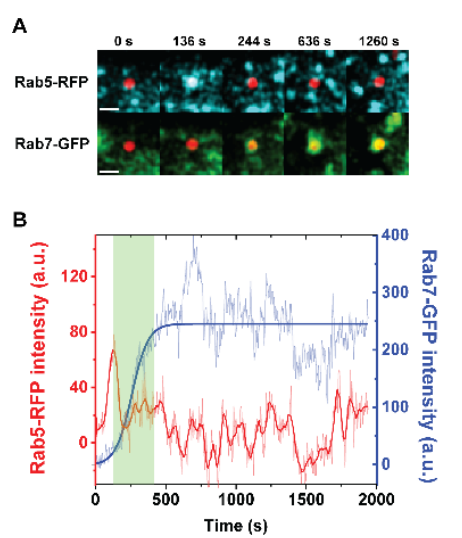

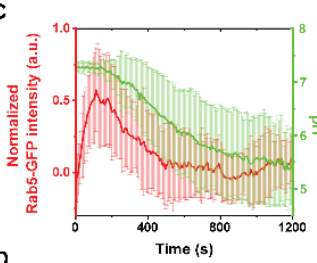

D

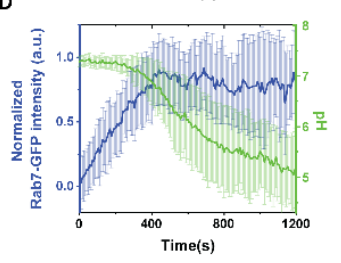

E
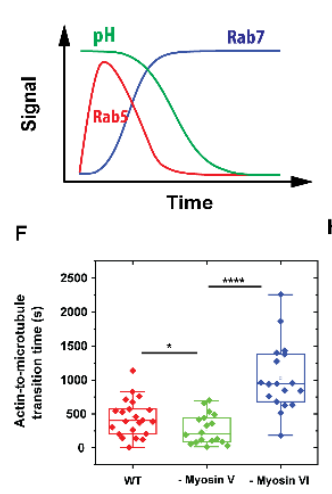

G
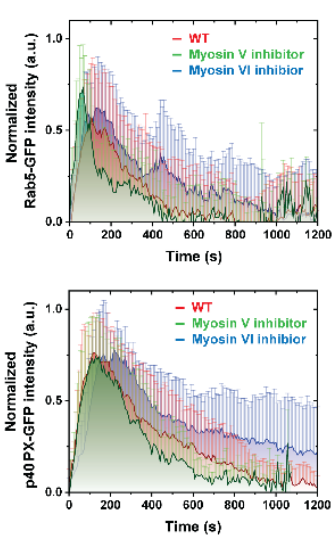

Figure 5. Dependence of early-to-late phagosome transition on actin-to-microtubule transport. (A-B) Fluorescence images and intensity plots showing the dynamic distribution of Rab5-RFP and Rab7-GFP around a representative phagosome during maturation in an activated RAW 264.7 macrophage cell. In the overlaid fluorescence images in (A), the pH-sensor containing phagosome is shown in red, Rab5-RFP in cyan and Rab7-GFP in green. Scale bar, $2 \mu \mathrm{m}$. The thick blue line indicates sigmoidal-Boltzmann function fitting to 
the Rab7-GFP data. The green shaded area indicates the period of Rab5-to-Rab7 transition from the first Rab5 intensity peak to the plateau of Rab7-GFP intensity. (C-D) Line plots showing average normalized intensity of Rab5-GFP (in C), Rab7-GFP (in D), and average normalized phagosome pH (in both plots) as a function of time. The line curves were averaged from 26 and 17 individual phagosomes in Rab5-RFP and Rab7-GFP expressing cells, respectively. Vertical bars indicate standard deviations. (E) A schematic showing the dynamic changes of Rab5, Rab7, and pH during singe phagosome acidification. (F) Scatter plot showing actin-to-microtubule transition time of single phagosomes with or without drug treatments as indicated. The average actin-to-microtubule transition time of phagosomes is $440 \pm 266 \mathrm{~s}$ in cells without drug treatment (WT), $276 \pm 214 \mathrm{~s}$ in cells with Myosin V inhibitor, and $1026 \pm 487 \mathrm{~s}$ in cells with Myosin VI inhibitor. In all scattered plots, each box plot indicates the mean (horizontal line) and the interquartile range from $25 \%$ to $75 \%$ of the corresponding data set. Statistical significance is highlighted by p-values (student's t-test) as follows: ${ }^{* * * *} p<0.0001,{ }^{*} p<0.05$. (G) Plots showing average normalized intensity of Rab5-GFP as a function of time with or without drug treatments as indicated. The line curves were averaged from 26, 10, and 16 individual phagosomes in cells without drug treatment (WT), with Myosin V inhibitor, and with Myosin VI inhibitor, respectively. Vertical bars represent standard deviations. (H) Plots showing the average normalized intensity of p40PX-GFP, a probe for PI3P, in cells under different drug treatment. The line curves are averaged from 12,14 , and 8 individual phagosomes without drug treatment (WT), with Myosin V inhibition, and with Myosin VI inhibition, respectively. Vertical bars represent standard deviations.

To determine the role of actin-based phagosome transport in phagosome maturation, we inhibited Myosin V and $\mathrm{VI}$ separately in cells and quantified their effect on the recruitment of Rab5. Myosin $\checkmark$ moves cargos along actin filaments from the minus end to the plus end, and was shown to tether endosomes and phagosomes strongly to the actin cortex and delay their transport onto microtubules (Al-Haddad et al., 2001; Kapitein et al., 2013; Lecuona et al., 2009; Maschi, Gramlich, \& Klyachko, 2018; Provance et al., 2008). Contrary to the case of Myosin V, Myosin VI transports cargos from the plus end of actin to the minus end (Wells et al., 1999). It facilitates cargo transport from actin to microtubules, as its inhibition causes phagosomes and endosomes to be entrapped in actin cortex (Aschenbrenner, Lee, \& Hasson, 2003; Aschenbrenner, Naccache, \& Hasson, 2004; Hewage \& Altman, 2018). Indeed, in cells treated with the Myosin V inhibitor MyoVin-1, phagosomes started microtubule-based rapid transport sooner than those in non-treated cells, with a shortened actin-to-microtubule transition time of $276 \pm 214 \mathrm{sec}$ (Figure 5F and Figure 5-figure supplement $4 \mathrm{~A}-\mathrm{C}$ ). On the contrary, phagosomes in cells treated with the Myosin VI inhibitor 2,4,6triiodophenol (TIP) remained in the actin-based slow-moving stage for a prolonged period of 1026 $\pm 487 \mathrm{sec}$ (Figure 5F and Figure 5-figure supplement 4E-G). The results confirm that inhibition of Myosin $\mathrm{V}$ promotes the handover of phagosomes from actin to microtubules, whereas inhibition of Myosin VI delays this transition. Correspondingly, we found that the duration of Rab5 association on phagosomes was shortened upon Myosin $\mathrm{V}$ inhibition, but prolonged upon Myosin VI inhibition (Figure 5G). We observed the similar effects of myosin inhibition on another early phagosome marker phosphatidylinositol 3-phosphate (PI3P), which is labeled with a genetically encoded biosensor p40PX-GFP. PI3P is a phospholipid generated after Rab5 recruitment by the class III phosphoinositide 3-kinase Vps34 and required for phagosome maturation (Gillooly, Simonsen, \& Stenmark, 2001). As shown in Figure $5 \mathrm{H}$, inhibition of Myosin $\mathrm{V}$ led to shorter duration of PI3P in the phagosomal membrane, whereas inhibition of Myosin VI caused the opposite effect. The observations from Rab5 and PI3P consistently show that a faster actin-to-microtubule transition with Myosin $\mathrm{V}$ inhibition shortens the duration over which Rab5 and PI3P are present on phagosomes. On the other hand, a slower actin-to-microtubule transition due to Myosin VI inhibition prolongs Rab5 and PI3P association with phagosomes. The results evidently show that the timing of transition of phagosomes from actin onto microtubules regulates the turnover of Rab5 and PI3P on phagosomes.

Because both Rab5 and PI3P are early phagosome markers and their turnover determines the progression of phagosome maturation (Chua \& Deretic, 2004; Jeschke et al., 2015; Kitano et al., 2008; Levin, Grinstein, \& Schlam, 2015; Schnatwinkel et al., 2004; Vieira et al., 2003), we postulated that the inhibition of Myosin V and VI should also affect phagosome acidification. Indeed, 
we found that the inhibition of Myosin $\mathrm{V}$ led to an earlier onset of rapid acidification ( $\mathrm{pH}$-standby time $371 \pm 200 \mathrm{sec})$. It also led to a faster acidification rate $(0.54 \pm 0.32 \mathrm{unit} / \mathrm{min})$ and a lower final $\mathrm{pH}(4.8 \pm 0.7)$, compared to non-treated cells ( $\mathrm{pH}$-standby time: $620 \pm 292 \mathrm{sec}$; acidification rate: $0.34 \pm 0.14$ unit/min; final pH: $5.5 \pm 0.8$ ) (Figure $6 \mathrm{~A}-\mathrm{C}$, Figure 1 -figure supplement $6 \mathrm{D}$ and Figure 5-figure supplement 4D). The opposite effect was observed with Myosin VI inhibition: phagosomes exhibited a delayed start of acidification ( $\mathrm{pH}$-standby time $718 \pm 323 \mathrm{sec}$ ), a slower acidification rate $(0.25 \pm 0.14 \mathrm{unit} / \mathrm{min})$, and a higher final $\mathrm{pH}(5.9 \pm 1.0)$. By plotting the $\mathrm{pH}$-standby time of single phagosomes against their actin-to-microtubule transition time (Figure 6D), we confirmed the positive correlation between the two variables. Phagosomes with shorter actin-tomicrotubule transition time overall had shorter $\mathrm{pH}$-standby time. It is therefore clear that the timing of phagosome transport from actin to microtubules regulates acidification. Phagosomes that moved faster from the actin cortex onto microtubules started acidification sooner, acidified more rapidly, and reached a lower final $\mathrm{pH}$. We have observed that the actin-to-microtubule transport of phagosomes regulates the turnover of Rab5 and PI3P, both of which are a prerequisite for phagosome acidification (Fratti, Backer, Gruenberg, Corvera, \& Deretic, 2001; Jeschke \& Haas, 2018; Law et al., 2017; Vergne et al., 2005). Thus, it is plausible that the actin-to-microtubule transition regulates phagosome acidification as a downstream consequence of regulating this turnover. Our results here demonstrate that the timing of actin-to-microtubule transport of phagosomes regulates the transient recruitment and dissociation of Rab5 and PI3P on phagosomes and therefore acts as a temporal control of the maturation of phagosomes, including their acidification.

A

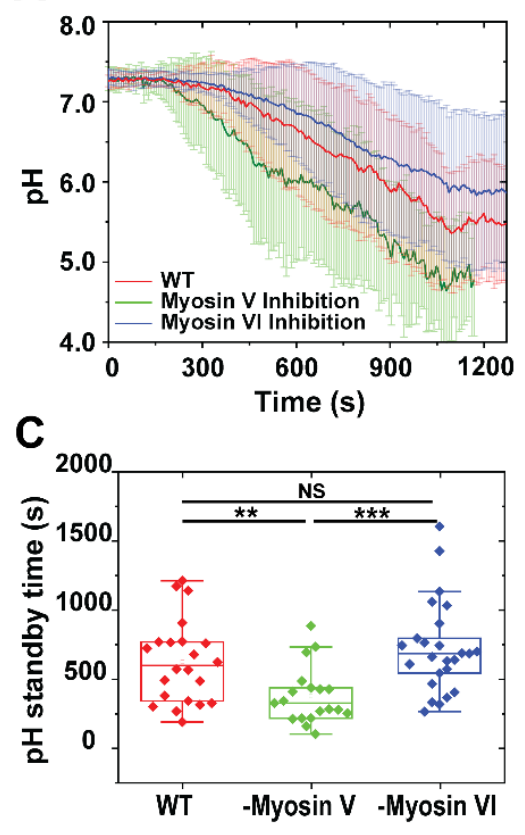

B

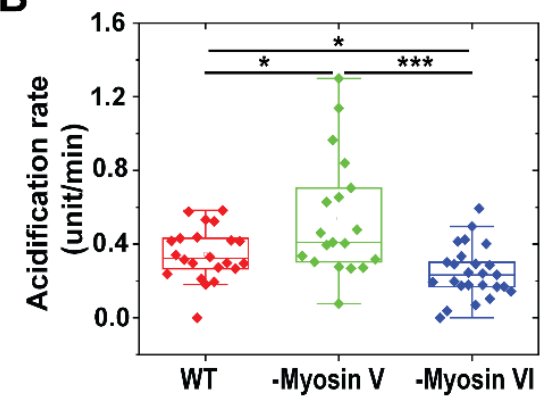

D

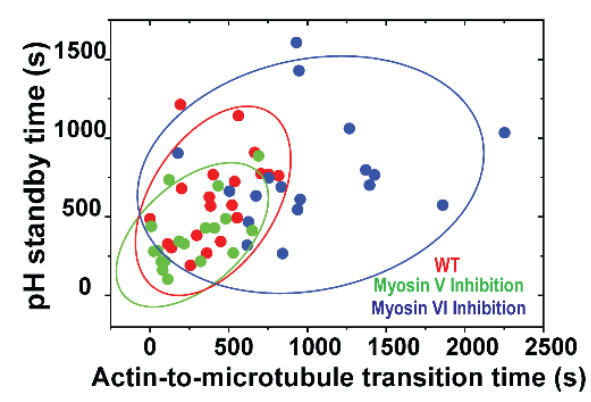

Figure 6. Dependence of phagosome acidification on actin-to-microtubule transport. (A) Line plots showing the average normalized $\mathrm{pH}$ as a function of time with or without drug treatments as indicated. The line curves are averaged from 22, 19, and 25 individual phagosomes in cells without drug treatment (wild type), with Myosin V inhibitor, and with Myosin VI inhibitor, respectively. Vertical bars represent standard deviations. (B) Scatter plot showing $\mathrm{pH}$ standby time of single phagosomes with or without drug treatments as indicated. The average $\mathrm{pH}$ standby time of phagosomes is $620 \pm 292 \mathrm{~s}$ without drug treatment (WT), $371 \pm 200 \mathrm{~s}$ with Myosin $\mathrm{V}$ inhibition, and $718 \pm 323 \mathrm{~s}$ with Myosin VI inhibition. (C) Scatter plot showing acidification rate of single phagosomes with or without drug treatments as indicated. The average acidification rate of phagosomes is 
$0.34 \pm 0.14 \mathrm{pH}$ unit/min in cells without drug treatment (WT), $0.54 \pm 0.32 \mathrm{pH}$ unit/min with Myosin V inhibition, and $0.25 \pm 0.14 \mathrm{pH}$ unit/min with Myosin VI inhibition. In all scatter plots, each box plot indicates the mean (horizontal line) and the interquartile range from $25 \%$ to $75 \%$ of the corresponding data set. Statistical significance is highlighted by $p$-values (student's t-test) as follows: ${ }^{*} p<0.05,{ }^{* *} p<0.01,{ }^{* * *} p<0.001$, NS $p$ $>0.05$. (D) Scatter plot showing $\mathrm{pH}$ standby time against actin-to-microtubule transition time of single phagosomes with or without drug treatments as indicated.

\section{Correlation between Rab5-to-Rab7 transition and phagosome transport velocity}

During the analysis of the Rab5 and Rab7 recruitment to phagosomes, we observed some correlation between the duration of the Rab5-to-Rab7 transition and the phagosome velocity that follows. Phagosomes from the same cell are compared in Figure 7A-B. The figure shows that phagosomes that had less Rab5 association (shorter duration and lower integrated intensity) and a faster transition to Rab7 moved faster during the microtubule-based transport and acidified more rapidly (Figure 7A-B). When we quantified the duration of the Rab5-to-Rab7 transition as the time from the initial Rab5 peak to the point when Rab7 intensity reaches a plateau (shaded area in Fig. $5 A$ ), we noticed that it showed an inverse correlation with the microtubule-based velocity of phagosomes with a Pearson's coefficient of -0.72 (Figure $7 \mathrm{C}$ ). These observations suggest that phagosomes with a faster Rab5-to-Rab7 conversion move faster afterwards on microtubules. However, we do not yet know the causal relationship between them. It is plausible that the two processes form a positive feedback loop, in which the efficient exchange of Rab5 by Rab7 facilitates phagosome transport on microtubules, which in turn promotes Rab7 recruitment.

A

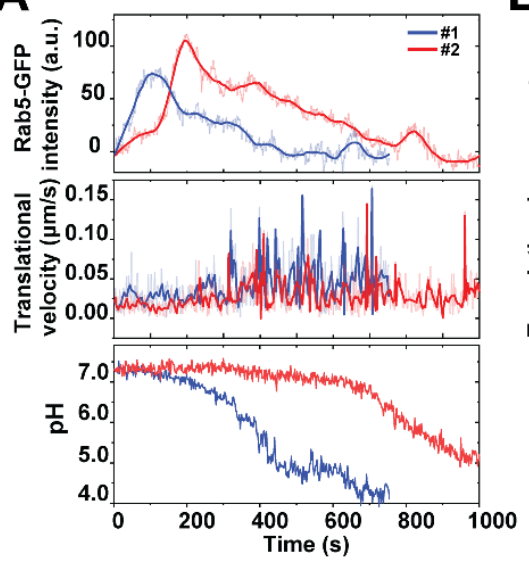

B

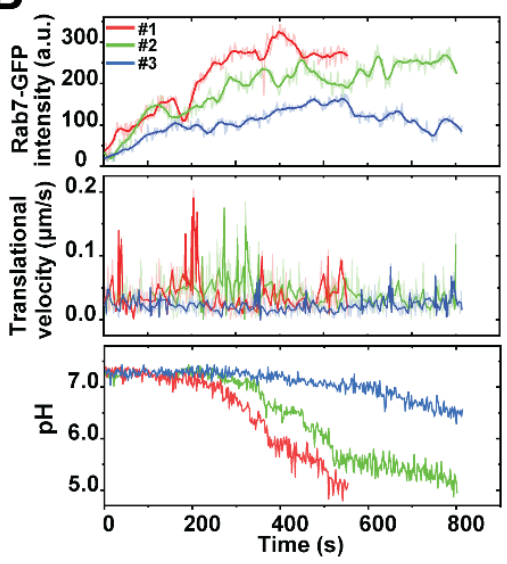

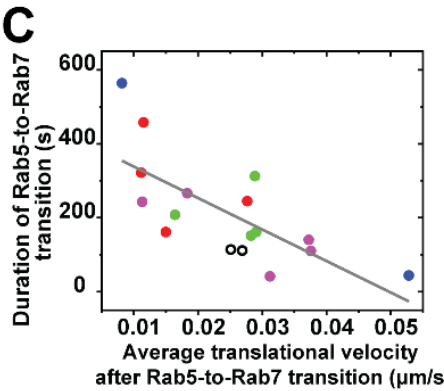

Figure 7. Rab5 and Rab7 recruitment and transport dynamics of single phagosomes from same cells. (A) Fluorescence intensity of Rab5-GFP, translational velocity, and phagosome lumen $\mathrm{pH}$ from three individual phagosomes within a same cell are plotted as a function of time. (B) Fluorescence intensity of Rab7-GFP, translational velocity, and phagosome lumen $\mathrm{pH}$ from three individual phagosomes within a same cell are plotted as a function of time. (C) Scatter plot showing the duration of Rab5-to-Rab7 conversion of individual phagosomes against their translational velocity after the Rab5-to-Rab7 conversion phase in activated RAW264.7 macrophage cells. Data points from multiple phagosomes within the same cells are shown in the same solid color. Data points from cells containing only one phagosome are shown as black circles. The linear regression has a Pearson's coefficient of -0.72 .

\section{Discussion}

The intracellular trafficking of phagosomes is known to be important for their maturation and, thus, for the effective degradation of pathogens. However, little is known about how the motility of phagosomes regulates the biochemical progression of phagosomes during maturation. In this 
study, we developed multi-functional particle sensors as phagocytic targets to combine biochemical imaging, tracking, and magnetic manipulation of single phagosomes in living cells. This integrated approach allowed us to discover two distinct mechanisms by which the intracellular trafficking of phagosomes regulates the timing of their maturation process. First, microtubule-dependent transport of phagosomes promotes their acquisition of late-phagosome and phagolysosomal characteristics. Their transport velocity directly regulates how fast they fuse with lysosomes, which in turn regulates how fast they acidify. However, it doesn't influence the final $\mathrm{pH}$ of the phagosomes. Second, phagosome transport from actin cortex to microtubules regulates the assembly of proteins to nascent phagosomes and the subsequent maturation progression. Timing of the actin-tomicrotubule transition regulates the duration of Rab5 and PI3P recruitment to phagosomes, which further influences the progression to late phagosomes (Rab7 recruitment) and the acidification. These two mechanisms are not exclusive of one another; in fact, they are interconnected. This is indicated by the observation that phagosomes with faster Rab5-to-Rab7 transition tend to be more mobile on microtubules in the late phagosome stage.

One of the key findings of our study is that the transport velocity of phagosomes on microtubules directly regulates phagosome fusion with lysosomes and consequently their acidification rate. It is known that phagosome fusion with lysosomes requires microtubule-dependent transport (Blocker et al., 1997; Desjardins et al., 1994; Dumas et al., 2015; Funato et al., 1997; Huynh et al., 2007; A. Rai et al., 2016; A. K. Rai, Rai, Ramaiya, Jha, \& Mallik, 2013; Sun-Wada et al., 2009). By fusion with lysosomes, phagosomes acquire proteins essential for maturation, including V-ATPase for acidification (Sun-Wada et al., 2009). However, unlike previous studies, our results reveal a causal and quantitative relationship between these processes. By simultaneously tracking the transport dynamics and maturation of single phagosomes, we first determined that more mobile phagosomes experience faster fusion with lysosomes and acidify faster. We then confirmed the causal nature of this relationship using magnetic tweezers experiments. In these experiments we showed that when phagosomes are accelerated, they speed up their fusion with lysosomes and their acidification. We further showed that the probability of productive phagosome-lysosome fusion is determined by the motility both of phagosomes, and also of lysosomes. Phagosomes failed to fuse with lysosomes or to acidify in cells with disrupted microtubules, even if phagosomes were moved magnetically. This finding demonstrates that the active transport, rather than simple diffusion, of phagosomes regulates phagosome-lysosome fusion and the acidification kinetics. Our results here do not exclude other possible mechanisms that might link the microtubule-based transport of phagosomes with the maturation kinetics. In fact, our observation that the rotational velocity of phagosomes correlates with the acidification rate suggests possible involvement of molecular motors. We have shown in previous studies that cargos rotate during transport on microtubules because of the "tugof-war" between molecular motors (Gao, Anthony, Yi, et al., 2018; Gao, Anthony, Yu, et al., 2018). Other studies have also shown that the Rab7-RILP (Rab7-interacting lysosomal protein) complex induces the recruitment of the dynein-dynactin motor protein complex to phagosomes (Johansson et al., 2007; Jordens et al., 2001), late endosomes and lysosomes (Harrison, Bucci, Vieira, Schroer, \& Grinstein, 2003) to regulate the retrograde transport of cargos. The Rab7-RILP complex also directly recruits the V-ATPase subunit V1G1 for phagosome maturation (Kissing et al., 2015). It is possible that the Rab7-RILP complexes function as the molecular link relating the phagosome motility with their acidification.

Surprisingly, we showed that the microtubule-based transport of phagosomes plays no role in determining the final $\mathrm{pH}$ of single phagosomes, which is an indicator of their degradative capacity. Instead, the final $\mathrm{pH}$ of a phagosome is affected by its transport within the actin cortex prior to moving onto microtubules. We found that, different from the microtubule-based transport, the period of phagosome transport from actin cortex onto microtubules regulates the initiation of phagosome maturation. Previous studies have shown that nascent phagosomes continue to acquire the molecules needed for maturation throughout their transition in the actin cortex (Heinsbroek et al., 2009; Kinchen et al., 2008; Yeung et al., 2009). In particular, proteins such as Rab5 and PI3P, 
which are required for the fusion of phagosomes with early endosomes (Duclos et al., 2000; Jeschke \& Haas, 2018), are recruited onto phagosome membranes before microtubule-based transport (Chua \& Deretic, 2004; Feliciano et al., 2011; Kitano et al., 2008). However, the role of actin in this process is unclear. Our results here demonstrate that the actin-to-microtubule translocation of phagosomes plays a much more significant role than merely proceeding coordinately with the early-to-late phagosome transition. We showed that prolonging the entrapment of phagosomes in actin cortex through Myosin $\mathrm{VI}$ inhibition significantly extended their early phagosome phase. This was indicated by the observation that the duration of Rab5 and PI3P association was prolonged and the onset of phagosome acidification was delayed. On the other hand, speeding up the transport of a phagosome from actin to microtubule transport through Myosin $V$ inhibition shortened the early phagosome phase and accelerated its transition to a late phagosome. Our findings reveal that the timing of phagosome transport from actin filaments to microtubules involves a mechanism that directly regulates early phagosome biogenesis, which then, in turn, regulates the subsequent cascades of phagosome maturation activities, including acidification. Several pathways exist by which the duration of phagosome entrapment in the actin cortex might control the assembly of Rab5 and PI3P onto phagosomes. Membrane-bound actin was shown in in vitro assays to facilitate the fusion of purified phagosomes with endosomes (Jahraus et al., 2001) and between purified yeast vacuoles (Eitzen, Wang, Thorngren, \& Wickner, 2002). The disruption of actin was also found to reduce the delivery of early endosomal content to pathogenic mycobacteria containing phagosomes (Guerin \& de Chastellier, 2000). Therefore, the retention of phagosomes in cortical actin might control the timing of their fusion with early endosomes to acquire proteins such as Rab5. Meanwhile, the timing of switching onto microtubules can also affect the delivery of Rab5-activating protein 6 (RAP6, also known as Gapex-5) (Kitano et al., 2008) and late endocytic content (Liebl \& Griffiths, 2009) to the phagosomes. This, in turn, affects the timing of Rab5-to-Rab7 conversion and phagosome maturation.

Another interesting observation of our study is that the duration of Rab5-to-Rab7 conversion is inversely correlated with the transport velocity of phagosomes on microtubules. Phagosomes with a shorter duration of Rab5 association and faster conversion to Rab7 move faster on microtubules after this conversion. We do not yet know what causal relationship might underlie this phenomenon. It is plausible that the two processes form a positive feedback loop. On the one hand, efficient replacement of Rab5 by Rab7 can facilitate phagosome transport on microtubules, as the Rab7RILP complex recruits the dynein-dynactin motor protein complex to phagosomes (Harrison et al., 2003). Rab7 also enhances phagosome-lysosome fusion by recruiting the homotypic fusion and vacuole protein sorting (HOPS) complex (Balderhaar \& Ungermann, 2013). It thus regulates acidification. On the other hand, interactions of phagosomes with non-motor microtubule associated proteins (MAPs) and MAP-binding proteins were also found to be essential for the activation of Rab GTPase (Feliciano et al., 2011; Kitano et al., 2008). This observation points out that despite the different roles of actin-based and microtubule-based transport in phagosome maturation, the underlying mechanisms are likely to be interconnected.

Individual phagosomes, even those from the same cells, exhibit a significant level of variation in maturation kinetics. There is no known mechanism that regulates the individual rate of phagosome maturation. Our results demonstrate that the intracellular transport of phagosomes functions as a maturation clock. Phagosome interaction with the cytoskeleton network can shape their degradative functions. This finding provides new insights into how pathogens might alter the subcellular environment in host cells, including the cytoskeletal structure and activities of molecular motors, to manipulate the degradative capacity of phagosomes as an immune-evasion strategy. 


\section{Materials and Methods}

\section{Materials}

Carboxylate-modified yellow-green fluorescent polystyrene nanoparticles (diameter $100 \mathrm{~nm}$ ), carboxylate-modified superparamagnetic Dynabeads (diameter $1 \mu \mathrm{m}$ ), eentylamine-biotin, Alexa Fluor 568 NHS ester (succinimidyl ester), Alexa Fluor 647 NHS ester (succinimidyl ester), pHrodo iFL Red STP Ester, Streptavidin, LysoTracker Green DND-26, Alexa Fluor 488 anti-tubulin- $\alpha$ antibody, Alexa Fluor Plus 647 phalloidin were purchased from ThermoFisher (Waltham, MA). Immunoglobulin $G$ from rabbit plasma, albumin from bovine serum (BSA), biotin $\mathrm{N}$ hydroxysuccinimide ester (biotin-NHS), nocodazole, lipopolysaccharides, MyoVin-1, and 2,4,6triiodophenol (TIP) were purchased from Sigma-Aldrich (St. Louis, MO). Monodisperse aminemodified silica particles (diameters $1.0 \mu \mathrm{m}$ ) were purchased from Spherotech Inc. (Lake Forest, IL). Sylgard 184 PDMS base was from Dow Corning (Midland, MI). 1-(3-Dimethylaminopropyl)-3ethylcarbodiimide hydrochloride (EDC) was purchased from Alfa Aesar (Haverhill, MA). Nigericin sodium salt was purchased from Tocris Bioscience (Minneapolis, MN). Recombinant Murine IFN- $\gamma$ was purchased from Peprotech (Rocky Hill, NJ). CF640R-amine was purchased from Biotium (Fremont, CA). FuGENE HD transfection reagent was purchased from Promega (Madison, WI). GFP-Rab5, RFP-Rab5, GFP-Rab7, and p40PX-GFP were prepared as previously described(Ellson et al., 2001; Kanai et al., 2001; Vieira et al., 2003). RAW264.7 macrophage cells were purchased from ATCC (Manassas, VA). RAW264.7 macrophages stably expressing EGFP-actin have been previously described(Scott et al., 2005). Ringer's solution $(\mathrm{pH}=7.2,10 \mathrm{mM}$ HEPES, $10 \mathrm{mM}$ glucose, $155 \mathrm{mM} \mathrm{NaCl}, 2 \mathrm{mM} \mathrm{NaH}_{2} \mathrm{PO}_{4}, 5 \mathrm{mM} \mathrm{KCl}, 2 \mathrm{mM} \mathrm{CaCl}$, $1 \mathrm{mM} \mathrm{MgCl}$ ) was used for livecell imaging. Potassium-rich solution (135 mM KCl, $2 \mathrm{mM} \mathrm{K}_{2} \mathrm{HPO}_{2}, 1.2 \mathrm{mM} \mathrm{CaCl}_{2}, 0.8 \mathrm{mM} \mathrm{MgSO}_{4}$ ) was used for intracellular $\mathrm{pH}$ calibration. Acidic washing solution $\left(135 \mathrm{mM} \mathrm{KCl}, 2 \mathrm{mM} \mathrm{K} \mathrm{HPO}_{2}, 1.2\right.$ $\mathrm{mM} \mathrm{CaCl}_{2}, 0.8 \mathrm{mM} \mathrm{MgSO}_{4}, 5 \mathrm{mM}$ sodium citrate) at $\mathrm{pH}$ of 4.5 was used for particle washing in the EDC coupling step of the RotSensor fabrication. Artificial lysosome fluid $(55 \mathrm{mM} \mathrm{NaCl}, 0.5 \mathrm{mM}$ $\mathrm{Na}_{2} \mathrm{HPO}_{4}, 0.26 \mathrm{mM}$ trisodium citrate dihydrate, $0.79 \mathrm{mM}$ glycine, $150 \mathrm{mM} \mathrm{NaOH}, 108 \mathrm{mM}$ citric acid, $0.87 \mathrm{mM} \mathrm{CaCl} \cdot 2 \mathrm{H}_{2} \mathrm{O}, 0.27 \mathrm{mM} \mathrm{Na}_{2} \mathrm{SO}_{4}, 0.25 \mathrm{mM} \mathrm{MgCl}_{2} \cdot 6 \mathrm{H}_{2} \mathrm{O}, 0.46 \mathrm{mM}$ disodium tartrate, $1.6 \mathrm{mM}$ sodium pyruvate) was prepared following a previously reported protocol (Pelfrene, Cave, Wragg, \& Douay, 2017) and used for washing particles during the protein conjugation step of the RotSensor fabrication.

\section{Cell Culture, Pharmacological Treatments, and Transfection}

All RAW264.7 cells were cultured in Dulbecco's Modified Eagle Medium (DMEM) complete medium supplemented with $10 \%$ fetal bovine serum (FBS), 100 units $/ \mathrm{ml}$ penicillin and $100 \mu \mathrm{g} / \mathrm{ml}$ streptomycin at $37^{\circ} \mathrm{C}$ and $5 \% \mathrm{CO}_{2}$. Resting RAW 264.7 cells were activated with a combination of $50 \mathrm{ng} / \mathrm{ml} \mathrm{LPS}$ and $100 \mathrm{units} / \mathrm{ml}$ IFN-y for $9 \mathrm{hr}$. For microtubule depolymerization, $10 \mu \mathrm{M}$ nocodazole was added to cells $0.5-1 \mathrm{hr}$ prior to live cell imaging and kept in the imaging buffer during live cell imaging. For Myosin inhibition, Myosin V inhibitor MyoVin-1 and Myosin 6 inhibitor 2,4,6triiodophenol (TIP) was separately dissolved in DMSO and added to cells $10 \mathrm{~min}$ before live cell imaging. The working concentration was $15 \mu \mathrm{M}$ for MyoVin-1 and $5 \mu \mathrm{M}$ for TIP. For cell transfection, 0.15 million RAW264.7 macrophage cells were seeded on a glass coverslip in $24 \mathrm{hr}$ prior to transfection. Transfection was carried out according to manufacturer's instructions. In brief, $500 \mathrm{ng}$ of plasmids and $1.5 \mu \mathrm{l}$ of FuGENE HD transfection reagents were mixed in $100 \mu \mathrm{l}$ DMEM and kept at room temperature for $15 \mathrm{~min}$. The transfection reagents were then added gently to the cells and incubated overnight before being removed.

\section{Fabrication and Characterization of Phagosome Sensors}




\subsection{Rotational Phagosome Sensors (RotSensors)}

pH-RotSensors: The first step was to covalently conjugate the amine-modified non-fluorescent silica particles $(1 \mu \mathrm{m})$ and the carboxylate-modified yellow-green fluorescent nanoparticles (100 $\mathrm{nm})$ at 1:1 ratio. Amine-modified non-fluorescent silica particles $(1 \mu \mathrm{m})$ were first incubated with $150 \mu \mathrm{g} / \mathrm{ml}$ biotin-NHS in $\mathrm{NaHCO}_{3}$ solution $(10 \mathrm{mM}, \mathrm{pH}$ 8.25) for $2 \mathrm{hr}$ at room temperature and then with $500 \mu \mathrm{g} / \mathrm{ml}$ of biotin-NHS for a second round of biotinylation. After biotinylation, the silica particles were washed in methanol and deionized (DI) water. Carboxylate-modified yellow-green fluorescent nanoparticles $(100 \mathrm{~nm})$ were mixed with the biotinylated amine-modified silica particles at a molar ratio of $333: 1$ in phosphate buffer $(10 \mathrm{mM}, \mathrm{pH} 7.0)$ containing $1.0 \mathrm{mg} / \mathrm{ml}$ EDC for the coupling. After $2 \mathrm{hr}$ incubation, particles were washed with acidic washing solution and then $1 \times$ PBS to remove the non-covalently bounded $100 \mathrm{~nm}$ particles. The second step was to conjugate fluorescently labeled streptavidin onto the RotSensors. Streptavidin-pHrodo Red conjugates (SAvpHrodo Red) were prepared according to manufacturer's instructions. To synthesize streptavidinCF640R conjugates (SAv-CF640), $0.7 \mathrm{mg}$ of streptavidin, $80 \mu \mathrm{g}$ of CF640R-amine, and $3 \mathrm{mg}$ of EDC were mixed in $350 \mu \mathrm{l}$ MES buffer $(50 \mathrm{mM}, \mathrm{pH} 4.5)$ for $3 \mathrm{hr}$. After the labeling, free dyes were removed by centrifugal filtration using Amicon Ultra filters (30K). RotSensors was then incubated with $25 \mu \mathrm{g} / \mathrm{ml}$ of SAv-pHrodo Red, $2.5 \mu \mathrm{g} / \mathrm{ml}$ of SAv-CF640R, $5 \mu \mathrm{g} / \mathrm{ml}$ of BSA and $1 \mu \mathrm{g} / \mathrm{ml}$ of IgG in $1 \times P B S$ for $5 \mathrm{hr}$ at room temperature. Unbound proteins were rinsed off with artificial lysosome solution and $1 \times$ PBS. The ratiometric $\mathrm{pH}-$ RotSensors were further opsonized with $30 \mu \mathrm{g} / \mathrm{ml} \mathrm{lgG}$ in $1 \times$ PBS for additional $2 \mathrm{hr}$ before live cell experiments.

FRET-RotSensors: Streptavidin-Alexa568 (SAv-Alexa 568) conjugates were first synthesized following manufacturer's instructions. The RotSensors were then incubated with $27.5 \mu \mathrm{g} / \mathrm{ml}$ of SAvAlexa $568,5 \mu \mathrm{g} / \mathrm{ml}$ of BSA, and $1 \mu \mathrm{g} / \mathrm{ml}$ of $\mathrm{lgG}$ in $1 \times P B S$ for $5 \mathrm{hr}$ at room temperature. Unbound proteins were rinsed off with artificial lysosome solution and $1 \times$ PBS. The FRET-RotSensors were further opsonized with $30 \mu \mathrm{g} / \mathrm{ml}$ of $\mathrm{IgG}$ in $1 \times$ PBS for additional $2 \mathrm{hr}$ before live cell experiments.

\subsection{Magnetically Modulated Phagosome Sensors (MagSensors)}

pH-MagSensors: For biotinylation, $10 \mu \mathrm{l}$ of magnetic particles $(1 \mathrm{mg} / \mathrm{ml})$ were rinsed three times with MES buffer (50 mM, pH 6.2) and resuspended in $100 \mu \mathrm{l}$ of MES buffer (50 mM, pH 6.2) containing $10 \mathrm{mg} / \mathrm{ml} \mathrm{EDC}$ and $1 \mathrm{mM}$ of biotin pentylamine. After 1-hr incubation at room temperature, particles were washed and resuspended into $100 \mu \mathrm{l}$ of $1 \times$ PBS. SAv-pHrodo Red and SAv-CF640R were then mixed with the MagSensors for $1 \mathrm{hr}$ at room temperature. The working concentration for SAv-pHrodo Red and SAv-CF640R was both $50 \mu \mathrm{g} / \mathrm{ml}$. Finally, particles were opsonized with $1 \mathrm{mg} / \mathrm{ml}$ lgG in $1 \times P B S$ for $1 \mathrm{hr}$ before live cell experiments.

FRET-MagSensors: Biotinylated magnetic particles were washed and resuspended in $1 \times$ PBS buffer containing $50 \mu \mathrm{g} / \mathrm{ml}$ of SAv-Alexa 568. The biotin-streptavidin conjugation was carried out for $1 \mathrm{~h}$ at room temperature. Particles were opsonized with $1 \mathrm{mg} / \mathrm{ml} \mathrm{IgG} \mathrm{in} 1 \times P B S$ for $1 \mathrm{hr}$ before live cell experiments.

\section{Magnetic Tweezers Setup and Force Calibration}

Magnetic tweezers were built on an inverted fluorescence microscope system (Nikon Eclipse Ti-U, Nikon, Tokyo, Japan), as shown in Figure 3-figure supplement 1A. The setup mainly includes a solenoid and a power supply. The solenoid was assembled by inserting a high permeability HyMu80 alloy rod (Carpenter Technology, Reading, PA) into an aluminum bobbin wrapped with 600 turns of copper coil (Zahn et al., 2017) (Figure 3-figure supplement 1B). The tip of the rod has a diameter of $\approx 1 \mu \mathrm{m}$ (Figure 3-figure supplement $1 \mathrm{C}$ ). Position of the solenoid was controlled with a manual micromanipulator (Narishige NMN-21) to achieve independent control of its position in 
the $x-, y-$, and z-direction with a minimum graduation of $250 \mathrm{~nm}$ in the $x$-y plane and $1 \mu \mathrm{m}$ in the $z-$ direction. The current going through the solenoid was generated by a programmable power supply (Tekpower, Montclair, CA) with a maximum power output of $5 \mathrm{~A}$.

Magnetic force was calibrated as a function of particle-to-tip distance by measuring the movements of single magnetic particles in the magnetic field. The particles were first conjugated with the CF640R dye via EDC-mediated amine-carboxyl coupling following the manufacturer's protocol, and then mixed in PDMS base at a concentration of $\approx 3.0 \times 10^{5} / \mathrm{ml}$. This low particle concentration was necessary to avoid particle aggregation and inter-particle magnetic inference (Etoc et al., 2013). The PDMS base, which has a high viscosity of $5.1 \mathrm{~Pa} \cdot \mathrm{s}$, was chosen to slow down particle movements to make particle tracking feasible. The initial positions of the solenoid tip and of the magnetic particles were imaged in bright field before time-elapse epi-fluorescence images of particles were acquired with an interval time of $0.2 \mathrm{~s}$. Working current of the magnetic tweezers was $1.0 \mathrm{~A}$ in the calibration. The relationship between magnetic force $F(r)$ and particle-to-tip distance $r$ was fitted using equation $F(r)=\frac{F_{0}}{\left[\frac{r}{2 r_{0}}+\frac{1}{2} C_{F}\right.}$, which has the property that $F\left(r_{0}\right)=F_{0}$. $F_{0}$ is a force constant with the unit of $\mathrm{pN}$; $r_{0}$ is a distance constant with the unit of $\mu \mathrm{m} ; \mathrm{C}_{\mathrm{F}}$ is unitless (Zahn et al., 2017).

\section{Fluorescence Microscopy}

5.1 Single-phagosome pH Assay. To synchronize particle uptake, cells were put on ice for $3 \mathrm{~min}$ before the addition of RotSensors and MagSensors, centrifuged at $200 \times \mathrm{g}$ at $4^{\circ} \mathrm{C}$ for $30 \mathrm{~s}$, and then incubated for $5 \mathrm{~min}$ at $37^{\circ} \mathrm{C}$ before live-cell imaging. Epi-fluorescence imaging was done using Nikon Eclipse-Ti inverted microscope equipped with a 1.64 N.A. $\times 100$ TIRF objective (Nikon, Tokyo, Japan) and an Andor iXon3 EMCCD camera (Andor Technology, Belfast, U.K.). Fluorescence emissions at three wavelengths (ex: 488, 561, and $640 \mathrm{~nm}$; em: 515, 586 and 680 $\mathrm{nm}$ ) were acquired for the time-lapse multi-channel imaging.

5.2 Single-phagosome FRET-fusion Assay. Cells were cultured in serum-containing DMEM supplemented with $5 \mu \mathrm{g} / \mathrm{ml}$ biotin-BSA-Alexa 647 overnight. For probing phagosome-lysosome fusion, biotin-BSA-Alexa 647 was chased in serum-containing DMEM $2 \mathrm{hr}$ before live cell imaging to ensure its accumulation in lysosome compartments, following previously reported protocols (Becken, Jeschke, Veltman, \& Haas, 2010; Jeschke \& Haas, 2018; Jeschke et al., 2015). Cells were then prepared the same way for synchronized particle uptake as single-phagosome $\mathrm{pH}$ assay. Time-lapse multi-channel epi-fluorescence images were acquired to record fluorescence emission of donor Alexa 568 (ex/em 561/586 nm), acceptor Alexa 647 (ex/em 561/680 nm), and the $100 \mathrm{~nm}$ green fluorescence particle (ex/em 488/515 nm). Image acquisition rate was $2 \mathrm{~s} /$ frame and 7 s/frame for RotSensors and MagSensors, respectively. A slight spectral overlap was observed that a low level of fluorescence emission of Alexa 568 under the excitation of $561 \mathrm{~nm}$ leaked into the acceptor emission channel $(680 \mathrm{~nm})$. Because this crosstalk was constant during imaging, it was measured and subtracted from the acceptor emission signal. Following previous protocol (Kaufmann, Goldman, \& Krise, 2009), the FRET ratio was calculated as the ratio of the emission intensity of acceptor after crosstalk subtraction $I_{\text {acceptor }}$ to the emission of intensity of the donor $I_{\text {donor }}$ at the donor excitation $(561 \mathrm{~nm})$. In the FRET-ratio-vs-time plot, the slope of the increment portion of the FRET ratio curve, named FRET rate, was used for the quantification of phagosomelysosome fusion rate (Figure 2-figure supplement 2).

5.3 Fluorescence Labeling and Imaging. RAW 264.7 macrophages were plated on $35 \mathrm{~mm}$ glass coverslips overnight before immunofluorescence staining. Cells were washed with $1 \times$ PBS and fixed with $2 \%$ PFA at room temperature for 5 min, permeabilized with $0.1 \%$ Triton $X-100$ at room temperature for $5 \mathrm{~min}$, blocked with $2 \%$ BSA at room temperature for $1 \mathrm{hr}$, and stained with $2 \mu \mathrm{g} / \mathrm{mL}$ 
Alexa Fluor 488 anti-tubulin- $\alpha$ antibody and $2 \mu \mathrm{g} / \mathrm{mL}$ Alexa Fluor Plus 647 phalloidin for $1 \mathrm{hr}$. Superresolution structured illumination microscopy (SIM) images of immunostained $\alpha$-tubulin and F-actin were acquired using a DeltaVision OMX SR imaging system equipped with a Olympus Plan Apo $60 \times / 1.42$ PSF objective and a sCMOS camera. To label lysosomes with biotin-BSA-Alexa 647 and Lysotracker, biotin-BSA-Alexa 647 was chased in RAW 264.7 macrophage for $1.5 \mathrm{hr}$. Cells were then incubated with $50 \mathrm{nM}$ Lysotracker Green DND-26 for $30 \mathrm{~min}$. After that, cells were rinsed twice with Ringer solution to remove the remaining dye and imaged using Re-scan Confocal Microscopy (RCM) equipped with a 1.64 N.A. $\times 100$ TIRF objective and ORCA-fusion CMOS camera.

\subsection{Images Analysis}

(1) Image Registration. Image registration was done to correct for the optical shift between different imaging channels. $100 \mathrm{~nm}$ TetraSpeck fluorescent particles (Ex/Em: 360/430 nm, 505/515 $\mathrm{nm}, 560 / 580 \mathrm{~nm}$, and $660 / 680 \mathrm{~nm}$ ) were adsorbed on polylysine-coated coverslips at a surface density of $\approx 0.10$ bead $/ \mu \mathrm{m}^{2}$ and used as markers for image registration. After sequential imaging of the marker particles in three channels (Ex: 488, 561, and $640 \mathrm{~nm}$ ), an affine transformation (in ImageJ) was applied to align particle localization maps from 488 and $640 \mathrm{~nm}$ channels (termed target images) to that from the $561 \mathrm{~nm}$ channel (reference image). A global mapping matrix was obtained to record all the transformation steps and used to apply the same operations to all images.

\section{(2) Single-Particle Localization and Intensity Determination.}

The centroids of single particles in epi-fluorescence images were localized using a Gaussian-based localization algorithm in the ImageJ plugin Trackmate. To measure the emission of CF640R and pHrodo Red on $\mathrm{pH}-\mathrm{RotSensor}$ and $\mathrm{pH}-$ MagSensor, pixel intensities within a radius of $2 \mu \mathrm{m}$ from the localized centroid of the particle were integrated and background-corrected using custom MATLAB algorithms. Same procedure was carried out for determining $I_{\text {donor }}$ and $I_{\text {acceptor }}$ during phagosome-lysosome fusion assay. To determine the localization uncertainties, RotSensors were immobilized on glass coverslips and imaged for 200 consecutive frames. Localization uncertainty was defined as the standard deviation of the tracked particle positions in $\mathrm{x}$ - and $\mathrm{y}$-coordinates. The localization uncertainties of the $1 \mu \mathrm{m}$ and $100 \mathrm{~nm}$ particles were determined to be $20.64 \pm 4.05 \mathrm{~nm}$ and $13.12 \pm 2.32 \mathrm{~nm}$, respectively (Figure 1 -figure supplement 11 ).

(3) Translational and Rotational Tracking Analysis of RotSensors. The single particle tracking analysis was done as described previously (Sanchez, Yi, \& Yu, 2017; Y. Q. Yu et al., 2018). Briefly, translational velocity was determined from the centroid location of the $1 \mu \mathrm{m}$ red fluorescent particle $\left(\mathrm{X}_{\text {red, }} \mathrm{Y}_{\text {red }}\right)$ as a function of time. For rotational tracking, a vector was drawn from the centroid of the $1 \mu \mathrm{m}$ red fluorescent particle $\left(\mathrm{X}_{\text {red, }}, \mathrm{Y}_{\text {red }}\right)$ to that of the $100 \mathrm{~nm}$ yellow-green particle $\left(\mathrm{X}_{\text {green }}, \mathrm{Y}_{\text {green }}\right)$ belonging to the same RotSensor. Orientation of the vector was obtained as the in-plane angle, $\varphi$, for the single RotSensor. The length of the vector was used to calculate the out-of-plane angle, $\theta$, using the equation below:

$$
\theta=\arcsin \left(\frac{d}{r}\right)
$$

where $d$ is the projection inter-particle distance between a pair of green and red dots in each given image, and $r$ is the physical inter-particle distance. Because $r$ varies slightly from one particle to another due to the size distribution, $r$ was obtained as the maximal projected inter-particle distance $d_{\max }$ when a RotSensor samples all possible orientations and the $d_{\max }$ was larger than $550 \mathrm{~nm}$. In cases when $d_{\max }<550 \mathrm{~nm}, r$ was set to $550 \mathrm{~nm}$. Rotational velocity was determined from the rotation matrix derived from $\varphi$ and $\theta$ at consecutive frames. 
(4) Intracellular $\mathrm{pH}$ Calibration. The intracellular $\mathrm{pH}$ calibration of $\mathrm{pH}-\mathrm{RotSensors}$ and $\mathrm{pH}-$ MagSensors was done following a previously published protocol (Naufer et al., 2018). Macrophages seeded on glass coverslips were pretreated with $10 \mu \mathrm{M}$ concanamycin in Ringer's solution for $10 \mathrm{~min}$ before the addition of particles and then incubated at $37^{\circ} \mathrm{C}$ for $10 \mathrm{~min}$ to promote particle phagocytosis. Cell medium was replaced with potassium-rich buffers at different $\mathrm{pH}$ values. All buffers contain $20 \mu \mathrm{M}$ of nigericin, but different buffering agent: $5 \mathrm{mM}$ sodium citrate for $\mathrm{pH} 4.5$, $5 \mathrm{mM}$ MES for $\mathrm{pH} 5.5$, and $5 \mathrm{mM}$ HEPES for $\mathrm{pH} 6.5$ and 7.3. The $\mathrm{pH}$ calibration was done from $\mathrm{pH}$ 7.3 to 4.5 . For each $\mathrm{pH}$, cells were rinsed twice with the correspondingly buffer and allowed to equilibrate for 5-10 min before image acquisition. Fluorescence emission of the pHrodo Red at 586 $\mathrm{nm}\left(I_{\text {pHrodoRed }}\right)$ and reference dye CF640 at $680 \mathrm{~nm}\left(I_{\text {ref }}\right)$ was obtained at various $\mathrm{pH}$ and background-corrected to obtain ratiometric $\mathrm{pH}$ calibration plots (Figure 1-figure supplement 2). In live cell imaging, $\mathrm{pH}$ calibration was done for individual internalized $\mathrm{pH}-$ RotSensors and $\mathrm{pH}$ MagSensors to eliminate the effect of particle-to-particle variation in their $\mathrm{pH}$ responses. In brief, the ratiometric emission of the $\mathrm{pH}$-RotSensors and $\mathrm{pH}$-MagSensors before acidification was obtained as $I_{\text {pHrodo }} / I_{\text {ref }}$ at $\mathrm{pH} 7.3$ in each cell sample. After live cell imaging, the same cell sample was incubated in $\mathrm{pH} 4.5$ potassium-rich $\mathrm{pH}$ calibration buffer containing $20 \mu \mathrm{M}$ of nigericin to obtain the ratiometric emission $\left(I_{p H r o d o} / I_{r e f}\right)$ at $\mathrm{pH} 4.5$. An internal calibration curve was generated and fit onto a linear function that relates the ratiometric emission $\left(I_{\text {pHrodo }} / I_{\text {ref }}\right)$ of the $\mathrm{pH}$-RotSensor and $\mathrm{pH}-\mathrm{MagSensor}$ at $\mathrm{pH} 4.5$ and $\mathrm{pH}$ 7.3. The resulting linear function was used to convert the ratiometic fluorescence measurements to luminal $\mathrm{pH}$ values for that series.

(5) Image Analysis of the Recruitment of Rab5, Rab7, actin, and PI3P marker. The accumulation of Rab5-GFP around the particle-containing phagosome was analyzed using Image J and MATLAB. The following protocol is written using Rab5-GFP as an example, but the same method was applied for analyzing other proteins including Rab5-RFP, actin-GFP, Rab7 GFP and p40PX-GFP. In brief, we first localized the centroid position of the particle in ImageJ. Two circular masks, one with a radius of $1 \mu \mathrm{m}$ and the other $1.5 \mu \mathrm{m}$, were applied and centered at the localized centroid of the particle (Figure 5-figure supplement 5). The average GFP intensity of all pixels located within the inner circular mask ( $1 \mu \mathrm{m}$ radius) was obtained as the signal, and the average fluorescence intensity of all pixels located between the inner and outer circular masks was obtained as the local cytosolic background. The average Rab5-GFP intensity recruited to the phagosome was then obtained by subtracting the cytosolic background from the signal. In order to compare the duration of Rab5-GFP recruitment between different phagosomes imaged in different cells (as in Figure 5C and Figure 5-figure supplement 1), we normalized the measured arbitrary intensities of Rab5-GFP to the obtained maximum intensity value in each cell. Same imaging analysis was applied to other proteins including Rab5-RFP, actin-GFP, Rab7-GFP and p40PX-GFP.

(6) Transmission Electron Microscopy. Cell samples for TEM imaging were prepared based on a previously reported protocol with slight modification (Sanchez et al., 2017). Particle sensors were incubated with cells for $50 \mathrm{~min}$ at $37^{\circ} \mathrm{C}$ before trypsinization. After centrifugation, the cell pellet was fixed on ice for $1 \mathrm{hr}$ by using a mixture of $2.5 \%(\mathrm{w} / \mathrm{v})$ glutaraldehyde and $1 \%(\mathrm{w} / \mathrm{v})$ osmium tetroxide in $1 \times$ PBS buffer. Subsequently, the pellet was stained in $0.5 \%(\mathrm{w} / \mathrm{v})$ uranyl acetate aqueous solution for $12 \mathrm{hr}$ on ice. Cell pellets were dehydrated sequentially in a series of ice-cold aqueous solutions containing $30 \%(\mathrm{v} / \mathrm{v}), 50 \%, 75 \%, 90 \%, 95 \%$ and $100 \%$ ethanol for 5 min each. Dehydration in $100 \%(\mathrm{v} / \mathrm{v})$ ethanol was repeated three times at room temperature. The dehydrated cell pellet was immersed sequentially in resin infiltration solutions that contain ethanol and Spurr's resin at various volume ratio (ethanol: resin, 2:1, 1:1 and 1:2) for 30 min each at room temperature. Cells were cured in $100 \%$ resin for $18 \mathrm{hr}$ at $65^{\circ} \mathrm{C}$ prior to microtome sectioning. Sections were stained with $2 \%(\mathrm{w} / \mathrm{v})$ uranyl acetate for $10 \mathrm{~min}$ before imaging. Samples were imaged with a JEOL JEM-1010 Transmission Electron Microscope (Electron Microscopy Center, Indiana University).

\section{Data Availability}


All data generated or analyzed during this study are included in the manuscript. Source data that were used to generate Figures 1D-I, 2B-F, 3B-F, 4A-B, and 5A-H, 6A-D and 7A-C have been deposited on Figshare (Y. Yu, 2021).

\section{Acknowledgments}

We thank Prof. Sergio Grinstein at University of Toronto for his helpful discussion. We thank Mr. Andy Alexander at the Electronic Instrument Services of Indiana University for assistance with the setup of magnetic tweezers. We thank Dr. Barry Stein at the IUB Electron Microscopy Imaging Center, Dr. Jim Power at the IUB Light Microscopy Imaging Center (LMIC), Dr. Yi Yi at the Nanoscale Characterization Center for assistance with instrument use. The development of singleparticle rotational tracking method was supported by the National Science Foundation, Division of Chemical, Bioengineering, Environmental, and Transport Systems under Award No. 1554078. All other research reported in this publication was supported by the National Institute of General Medical Sciences of the NIH under Award No. R35GM124918 (Y. Y.). G.F.W.W. is supported by a Vanier Canada Graduate Scholarship (CIHR). The content is solely the responsibility of the authors and does not necessarily represent the official views of the $\mathrm{NIH}$.

\section{References}

Al-Haddad, A. H., Shonn, M. A., Redlich, B., Blocker, A., Burkhardt, J. K., Yu, H., . . Kuznetsov, S. A. (2001). Myosin va bound to phagosomes binds to F-actin and delays microtubuledependent motility. Molecular Biology of the Cell, 12(9), 2742-2755. doi:DOI $10.1091 / \mathrm{mbc} .12 .9 .2742$

Araki, N. (2006). Role of microtubules and myosins in Fc gamma receptor-mediated phagocytosis. Frontiers in Bioscience, 11, 1479-1490. doi:Doi 10.2741/1897

Arora, P. D., Manolson, M. F., Downey, G. P., Sodek, J., \& McCulloch, C. A. (2000). A novel model system for characterization of phagosomal maturation, acidification, and intracellular collagen degradation in fibroblasts. Journal of Biological Chemistry, 275(45), 35432-35441. doi:10.1074/jbc.M003221200

Arppe, R., Nareoja, T., Nylund, S., Mattsson, L., Koho, S., Rosenholm, J. M., . . Schaferling, M. (2014). Photon upconversion sensitized nanoprobes for sensing and imaging of $\mathrm{pH}$. Nanoscale, 6(12), 6837-6843. doi:10.1039/c4nr00461b

Aschenbrenner, L., Lee, T., \& Hasson, T. (2003). Myo6 facilitates the translocation of endocytic vesicles from cell peripheries. Molecular Biology of the Cell, 14(7), 2728-2743. doi:10.1091/mbc.E02-11-0767

Aschenbrenner, L., Naccache, S. N., \& Hasson, T. (2004). Uncoated endocytic vesicles require the unconventional myosin, Myo6, for rapid transport through actin barriers. Molecular Biology of the Cell, 15(5), 2253-2263. doi:10.1091/mbc.E04-01-0002

Balderhaar, H. J. K., \& Ungermann, C. (2013). CORVET and HOPS tethering complexes coordinators of endosome and lysosome fusion. Journal of Cell Science, 126(6), 13071316. doi:10.1242/jcs.107805 
Becken, U., Jeschke, A., Veltman, K., \& Haas, A. (2010). Cell-free fusion of bacteria-containing phagosomes with endocytic compartments. Proc Natl Acad Sci U S A, 107(48), 2072620731. doi:10.1073/pnas.1007295107

Blocker, A., Severin, F. F., Burkhardt, J. K., Bingham, J. B., Yu, H. R., Olivo, J. C., . . . Griffiths, G. (1997). Molecular requirements for bi-directional movement of phagosomes along microtubules. Journal of Cell Biology, 137(1), 113-129. doi:DOI 10.1083/jcb.137.1.113

Blocker, A., Severin, F. F., Habermann, A., Hyman, A. A., Griffiths, G., \& Burkhardt, J. K. (1996). Microtubule-associated protein-dependent binding of phagosomes to microtubules. Journal of Biological Chemistry, 271(7), 3803-3811.

Canton, J., Khezri, R., Glogauer, M., \& Grinstein, S. (2014). Contrasting phagosome pH regulation and maturation in human M1 and M2 macrophages. Molecular Biology of the Cell, 25(21), 3330-3341. doi:10.1091/mbc.E14-05-0967

Chua, J., \& Deretic, V. (2004). Mycobacterium tuberculosis reprograms waves of phosphatidylinositol 3-phosphate on phagosomal organelles. Journal of Biological Chemistry, 279(35), 36982-36992. doi:10.1074/jbc.M405082200

Claus, V., Jahraus, A., Tjelle, T., Berg, T., Kirschke, H., Faulstich, H., \& Griffiths, G. (1998). Lysosomal enzyme trafficking between phagosomes, endosomes, and lysosomes in $\mathrm{J774}$ macrophages enrichment of cathepsin $\mathrm{H}$ in early endosomes. Journal of Biological Chemistry, 273(16), 9842-9851.

Cossart, P., \& Helenius, A. (2014). Endocytosis of viruses and bacteria. Cold Spring Harbor Perspectives in Biology, 6(8). doi:10.1101/cshperspect.a016972

Deriy, L. V., Gomez, E. A., Zhang, G. P., Beacham, D. W., Hopson, J. A., Gallan, A. J., . . Nelson, D. J. (2009). Disease-causing Mutations in the Cystic Fibrosis Transmembrane Conductance Regulator Determine the Functional Responses of Alveolar Macrophages. Journal of Biological Chemistry, 284(51), 35926-35938. doi:10.1074/jbc.M109.057372

Desjardins, M., Huber, L. A., Parton, R. G., \& Griffiths, G. (1994). Biogenesis of Phagolysosomes Proceeds through a Sequential Series of Interactions with the Endocytic Apparatus. Journal of Cell Biology, 124(5), 677-688. doi:DOI 10.1083/jcb.124.5.677

Duclos, S., Diez, R., Garin, J., Papadopoulou, B., Descoteaux, A., Stenmark, H., \& Desjardins, M. (2000). Rab5 regulates the kiss and run fusion between phagosomes and endosomes and the acquisition of phagosome leishmanicidal properties in RAW 264.7 macrophages. Journal of Cell Science, 113(19), 3531-3541.

Dumas, A., Le-Bury, G., Marie-Anis, F., Herit, F., Mazzolini, J., Guilbert, T., . . N Niedergang, F. (2015). The HIV-1 protein Vpr impairs phagosome maturation by controlling microtubuledependent trafficking. Journal of Cell Biology, 211(2), 359-372. doi:10.1083/jcb.201503124

Eitzen, G., Wang, L., Thorngren, N., \& Wickner, W. (2002). Remodeling of organelle-bound actin is required for yeast vacuole fusion. Journal of Cell Biology, 158(4), 669-679. doi:10.1083/jcb.200204089

Ellson, C. D., Gobert-Gosse, S., Anderson, K. E., Davidson, K., Erdjument-Bromage, H., Tempst, P., . . Stephens, L. R. (2001). Ptdlns(3)P regulates the neutrophil oxidase complex by 
binding to the PX domain of p40(phox). Nature Cell Biology, 3(7), 679-682. doi: $10.1038 / 35083076$

Etoc, F., Lisse, D., Bellaiche, Y., Piehler, J., Coppey, M., \& Dahan, M. (2013). Subcellular control of Rac-GTPase signalling by magnetogenetic manipulation inside living cells. Nat Nanotechnol, 8(3), 193-198. doi:10.1038/nnano.2013.23

Feliciano, W. D., Yoshida, S., Straight, S. W., \& Swanson, J. A. (2011). Coordination of the Rab5 Cycle on Macropinosomes. Traffic, 12(12), 1911-1922. doi:10.1111/j.16000854.2011.01280.x

Fratti, R. A., Backer, J. M., Gruenberg, J., Corvera, S., \& Deretic, V. (2001). Role of phosphatidylinositol 3-kinase and Rab5 effectors in phagosomal biogenesis and mycobacterial phagosome maturation arrest. Journal of Cell Biology, 154(3), 631-644. doi:DOI 10.1083/jcb.200106049

Funato, K., Beron, W., Yang, C. Z., Mukhopadhyay, A., \& Stahl, P. D. (1997). Reconstitution of phagosome-lysosome fusion in streptolysin O-permeabilized cells. Journal of Biological Chemistry, 272(26), 16147-16151. doi:DOI 10.1074/jbc.272.26.16147

Gao, Y., Anthony, S. M., Yi, Y., Li, W., Yu, Y., \& Yu, Y. (2018). Single-Janus Rod Tracking Reveals the "Rock-and-Roll" of Endosomes in Living Cells. Langmuir, 34(3), 1151-1158. doi:10.1021/acs.langmuir.7b02804

Gao, Y., Anthony, S. M., Yu, Y. Q., Yi, Y., \& Yu, Y. (2018). Cargos Rotate at Microtubule Intersections during Intracellular Trafficking. Biophysical Journal, 114(12), 2900-2909. doi:10.1016/j.bpj.2018.05.010

Gibbs, D., Azarian, S. M., Lillo, C., Kitamoto, J., Klomp, A. E., Steel, K. P., . . Williams, D. S. (2004). Role of myosin VIla and Rab27a in the motility and localization of RPE melanosomes. Journal of Cell Science, 117(26), 6473-6483. doi:10.1242/jcs.01580

Gibbs, D., Kitamoto, J., \& Williams, D. S. (2003). Abnormal phagocytosis by retinal pigmented epithelium that lacks myosin Vila, the Usher syndrome 1B protein. Proceedings of the National Academy of Sciences of the United States of America, 100(11), 6481-6486. doi:10.1073/pnas.1130432100

Gillooly, D. J., Simonsen, A., \& Stenmark, H. (2001). Phosphoinositides and phagocytosis. Journal of Cell Biology, 155(1), 15-17. doi:DOI 10.1083/jcb.200109001

Granger, E., McNee, G., Allan, V., \& Woodman, P. (2014). The role of the cytoskeleton and molecular motors in endosomal dynamics. Seminars in Cell \& Developmental Biology, 31, 20-29. doi:10.1016/j.semcdb.2014.04.011

Guerin, I., \& de Chastellier, C. (2000). Disruption of the actin filament network affects delivery of endocytic contents marker to phagosomes with early endosome characteristics: The case of phagosomes with pathogenic mycobacteria. European Journal of Cell Biology, 79(10), 735-749. doi:Doi 10.1078/0171-9335-00092

Harrison, R. E., Bucci, C., Vieira, O. V., Schroer, T. A., \& Grinstein, S. (2003). Phagosomes fuse with late endosomes and/or lysosomes by extension of membrane protrusions along 
microtubules: role of Rab7 and RILP. Molecular and Cellular Biology, 23(18), 6494-6506. doi:10.1128/mcb.23.18.6494-6506.2003

Harrison, R. E., \& Grinstein, S. (2002). Phagocytosis and the microtubule cytoskeleton. Biochemistry and Cell Biology, 80(5), 509-515. doi:10.1139/O02-142

Heinsbroek, S. E. M., Kamen, L. A., Taylor, P. R., Brown, G. D., Swanson, J., \& Gordon, S. (2009). Actin and Phosphoinositide Recruitment to Fully Formed Candida albicans Phagosomes in Mouse Macrophages. Journal of Innate Immunity, 1(3), 244-253. doi:10.1159/000173694

Hendricks, A. G., Holzbaur, E. L., \& Goldman, Y. E. (2012). Force measurements on cargoes in living cells reveal collective dynamics of microtubule motors. Proceedings of the National Academy of Sciences of the United States of America, 109(45), 18447-18452. doi:10.1073/pnas.1215462109

Hewage, N., \& Altman, D. (2018). A role for myosin VI in retinal pigment epithelium phagocytosis. Biochemical and Biophysical Research Communications, 504(4), 759-764. doi:10.1016/j.bbrc.2018.09.006

Huang, N. N., Becker, S., Boularan, C., Kamenyeva, O., Vural, A., Hwang, I. Y., . . Kehrl, J. H. (2014). Canonical and Noncanonical G-Protein Signaling Helps Coordinate Actin Dynamics To Promote Macrophage Phagocytosis of Zymosan. Molecular and Cellular Biology, 34(22), 4186-4199. doi:10.1128/Mcb.00325-14

Huynh, K. K., Eskelinen, E. L., Scott, C. C., Malevanets, A., Saftig, P., \& Grinstein, S. (2007). LAMP proteins are required for fusion of lysosomes with phagosomes. EMBO Journal, 26(2), 313324. doi:10.1038/sj.emboj.7601511

Jahraus, A., Egeberg, M., Hinner, B., Habermann, A., Sackman, E., Pralle, A., . . G Giffiths, G. (2001). ATP-dependent membrane assembly of F-actin facilitates membrane fusion. Molecular Biology of the Cell, 12(1), 155-170. doi:DOI 10.1091/mbc.12.1.155

Jeschke, A., \& Haas, A. (2018). Sequential actions of phosphatidylinositol phosphates regulate phagosome-lysosome fusion. Molecular Biology of the Cell, 29(4), 452-465. doi:10.1091/mbc.E17-07-0464

Jeschke, A., Zehethofer, N., Lindner, B., Krupp, J., Schwudke, D., Haneburger, I., . . Haas, A. (2015). Phosphatidylinositol 4-phosphate and phosphatidylinositol 3-phosphate regulate phagolysosome biogenesis. Proceedings of the National Academy of Sciences of the United States of America, 112(15), 4636-4641. doi:10.1073/pnas.1423456112

Jiang, C., Lionberger, T. A., Wiener, D. M., \& Meyhofer, E. (2016). Electromagnetic tweezers with independent force and torque control. Review of Scientific Instruments, 87(8), 084304. doi:10.1063/1.4960811

Jiang, M., Esteve-Rudd, J., Lopes, V. S., Diemer, T., Lillo, C., Rump, A., \& Williams, D. S. (2015). Microtubule motors transport phagosomes in the RPE, and lack of KLC1 leads to AMD-like pathogenesis. Journal of Cell Biology, 210(4), 595-611. doi:10.1083/jcb.201410112

Johansson, M., Rocha, N., Zwart, W., Jordens, I., Janssen, L., Kuijl, C., . . Neefjes, J. (2007). Activation of endosomal dynein motors by stepwise assembly of Rab7-RILP-p150(Glued), 
ORP1L, and the receptor beta III spectrin. Journal of Cell Biology, 176(4), 459-471. doi:10.1083/jcb.200606077

Jordens, I., Fernandez-Borja, M., Marsman, M., Dusseljee, S., Janssen, L., Calafat, J., . . Neefjes, J. (2001). The Rab7 effector protein RILP controls lysosomal transport by inducing the recruitment of dynein-dynactin motors. Current Biology, 11(21), 1680-1685. doi:Doi 10.1016/S0960-9822(01)00531-0

Kagan, J. C., \& Roy, C. R. (2002). Legionella phagosomes intercept vesicular traffic from endoplasmic reticulum exit sites. Nature Cell Biology, 4(12), 945-954. doi:10.1038/ncb883

Kanai, F., Liu, H., Field, S. J., Akbary, H., Matsuo, T., Brown, G. E., . . Yaffe, M. B. (2001). The PX domains of p47phox and p40phox bind to lipid products of $\mathrm{PI}(3) \mathrm{K}$. Nature Cell Biology, 3(7), 675-678. doi:10.1038/35083070

Kapitein, L. C., van Bergeijk, P., Lipka, J., Keijzer, N., Wulf, P. S., Katrukha, E. A., . . Hoogenraad, C. C. (2013). Myosin-V Opposes Microtubule-Based Cargo Transport and Drives Directional Motility on Cortical Actin. Current Biology, 23(9), 828-834. doi:10.1016/j.cub.2013.03.068

Kaufmann, A. M., Goldman, S. D., \& Krise, J. P. (2009). A fluorescence resonance energy transferbased approach for investigating late endosome-lysosome retrograde fusion events. Analytical Biochemistry, 386(1), 91-97. doi:10.1016/j.ab.2008.11.036

Kelleher, J. F., \& Titus, M. A. (1998). Intracellular motility: How can we all work together? Current Biology, 8(11), R394-R397. doi:Doi 10.1016/S0960-9822(98)70246-5

Kinchen, J. M., Doukoumetzidis, K., Almendinger, J., Stergiou, L., Tosello-Trampont, A., Sifri, C. D., . . . Ravichandran, K. S. (2008). A pathway for phagosome maturation during engulfment of apoptotic cells. Nature Cell Biology, 10(5), 556-566. doi:10.1038/ncb1718

Kinchen, J. M., \& Ravichandran, K. S. (2008). Phagosome maturation: going through the acid test. Nature Reviews: Molecular Cell Biology, 9(10), 781-795. doi:10.1038/nrm2515

Kissing, S., Hermsen, C., Repnik, U., Nesset, C. K., von Bargen, K., Griffiths, G., . . Saftig, P. (2015). Vacuolar ATPase in Phagosome-Lysosome Fusion. Journal of Biological Chemistry, 290(22), 14166-14180. doi:10.1074/jbc.M114.628891

Kitano, M., Nakaya, M., Nakamura, T., Nagata, S., \& Matsuda, M. (2008). Imaging of Rab5 activity identifies essential regulators for phagosome maturation. Nature, 453(7192), 241-U215. doi:10.1038/nature06857

Law, F., Seo, J. H., Wang, Z. Q., DeLeon, J. L., Bolis, Y., Brown, A., . . Rocheleau, C. E. (2017). The VPS34 PI3K negatively regulates RAB-5 during endosome maturation. Journal of Cell Science, 130(12), 2007-2017. doi:10.1242/jcs.194746

Lecuona, E., Minin, A., Trejo, H. E., Chen, J. W., Comellas, A. P., Sun, H. Y., . . Sznajder, J. I. (2009). Myosin-Va restrains the trafficking of $\mathrm{Na}+/ \mathrm{K}+-\mathrm{ATP}$ ase-containing vesicles in alveolar epithelial cells. Journal of Cell Science, 122(21), 3915-3922. doi:10.1242/jcs.046953 
Lennon-Duménil, A.-M., Bakker, A. H., Maehr, R., Fiebiger, E., Overkleeft, H. S., Rosemblatt, M., ... Lagaudrière-Gesbert, C. (2002). Analysis of protease activity in live antigen-presenting cells shows regulation of the phagosomal proteolytic contents during dendritic cell activation. The Journal of experimental medicine, 196(4), 529-540.

Levin, R., Grinstein, S., \& Schlam, D. (2015). Phosphoinositides in phagocytosis and macropinocytosis. Biochimica et Biophysica Acta (BBA) - Molecular and Cell Biology of Lipids, 1851(6), 805-823. doi:10.1016/j.bbalip.2014.09.005

Liebl, D., \& Griffiths, G. (2009). Transient assembly of F-actin by phagosomes delays phagosome fusion with lysosomes in cargo-overloaded macrophages. Journal of Cell Science, 122(16), 2935-2945. doi:10.1242/jcs.048355

Maschi, D., Gramlich, M. W., \& Klyachko, V. A. (2018). Myosin V functions as a vesicle tether at the plasma membrane to control neurotransmitter release in a central synapses. Elife, 7. doi:ARTN e39440

\subsection{4/eLife.39440}

Mcneil, P. L., Tanasugarn, L., Meigs, J. B., \& Taylor, D. L. (1983). Acidification of Phagosomes Is Initiated before Lysosomal-Enzyme Activity Is Detected. Journal of Cell Biology, 97(3), 692702. doi:DOI 10.1083/jcb.97.3.692

Mottola, G. (2014). The complexity of Rab5 to Rab7 transition guarantees specificity of pathogen subversion mechanisms. Frontiers in Cellular and Infection Microbiology, 4. doi:ARTN 180

10.3389/fcimb.2014.00180

Naufer, A., Hipolito, V. E. B., Ganesan, S., Prashar, A., Zaremberg, V., Botelho, R. J., \& Terebiznik, M. R. (2018). pH of endophagosomes controls association of their membranes with Vps34 and Ptdlns(3)P levels. Journal of Cell Biology, 217(1), 329-346. doi:10.1083/jcb.201702179

Nunes, P., Guido, D., \& Demaurex, N. (2015). Measuring Phagosome pH by Ratiometric Fluorescence Microscopy. Jove-Journal of Visualized Experiments(106). doi:ARTN e53402

\section{$10.3791 / 53402$}

Oberhofer, A., Reithmann, E., Spieler, P., Stepp, W. L., Zimmermann, D., Schmid, B., . . Okten, Z. (2020). Molecular underpinnings of cytoskeletal cross-talk. Proceedings of the National Academy of Sciences of the United States of America, 117(8), 3944-3952. doi:10.1073/pnas.1917964117

Ogawa, M., Kosaka, N., Regino, C. A. S., Mitsunaga, M., Choyke, P. L., \& Kobayashi, H. (2010). High sensitivity detection of cancer in vivo using a dual-controlled activation fluorescent imaging probe based on $\mathrm{H}$-dimer formation and $\mathrm{pH}$ activation. Molecular Biosystems, 6(5), 888-893. doi:10.1039/b917876g

Pauwels, A. M., Trost, M., Beyaert, R., \& Hoffmann, E. (2017). Patterns, Receptors, and Signals: Regulation of Phagosome Maturation. Trends in Immunology, 38(6), 407-422. doi:10.1016/j.it.2017.03.006 
Pelfrene, A., Cave, M. R., Wragg, J., \& Douay, F. (2017). In Vitro Investigations of Human Bioaccessibility from Reference Materials Using Simulated Lung Fluids. International Journal of Environmental Research and Public Health, 14(2). doi:10.3390/ijerph14020112

Poteryaev, D., Datta, S., Ackema, K., Zerial, M., \& Spang, A. (2010). Identification of the Switch in Early-to-Late Endosome Transition. Cell, 141(3), 497-508. doi:10.1016/j.cell.2010.03.011

Provance, D. W., Addison, E. J., Wood, P. R., Chen, D. Z., Silan, C. M., \& Mercer, J. A. (2008). Myosin- $\mathrm{Vb}$ functions as a dynamic tether for peripheral endocytic compartments during transferrin trafficking. Bmc Cell Biology, 9. doi:Artn 44

$10.1186 / 1471-2121-9-44$

Rai, A., Pathak, D., Thakur, S., Singh, S., Dubey, A. K., \& Mallik, R. (2016). Dynein Clusters into Lipid Microdomains on Phagosomes to Drive Rapid Transport toward Lysosomes. Cell, 164(4), 722-734. doi:10.1016/j.cell.2015.12.054

Rai, A. K., Rai, A., Ramaiya, A. J., Jha, R., \& Mallik, R. (2013). Molecular Adaptations Allow Dynein to Generate Large Collective Forces inside Cells. Cell, 152(1-2), 172-182. doi:10.1016/j.cell.2012.11.044

Riazanski, V., Gabdoulkhakova, A. G., Boynton, L. S., Eguchi, R. R., Deriy, L. V., Hogarth, D. K., .. . Nelson, D. J. (2015). TRPC6 channel translocation into phagosomal membrane augments phagosomal function. Proceedings of the National Academy of Sciences of the United States of America, 112(47), E6486-E6495. doi:10.1073/pnas.1518966112

Rink, J., Ghigo, E., Kalaidzidis, Y., \& Zerial, M. (2005). Rab conversion as a mechanism of progression from early to late endosomes. Cell, 122(5), 735-749. doi:10.1016/j.cell.2005.06.043

Rybicka, J. M., Balce, D. R., Chaudhuri, S., Allan, E. R. O., \& Yates, R. M. (2012). Phagosomal proteolysis in dendritic cells is modulated by NADPH oxidase in a pH-independent manner. Embo Journal, 31(4), 932-944. doi:10.1038/emboj.2011.440

Sanchez, L., Yi, Y., \& Yu, Y. (2017). Effect of partial PEGylation on particle uptake by macrophages. Nanoscale, 9(1), 288-297. doi:10.1039/c6nr07353k

Savina, A., Jancic, C., Hugues, S., Guermonprez, P., Vargas, P., Moura, I. C., . . Amigorena, S. (2006). NOX2 controls phagosomal $\mathrm{pH}$ to regulate antigen processing during crosspresentation by dendritic cells. Cell, 126(1), 205-218.

Schnatwinkel, C., Christoforidis, S., Lindsay, M. R., Uttenweiler-Joseph, S., Wilm, M., Parton, R. G., \& Zerial, M. (2004). The Rab5 effector rabankyrin-5 regulates and coordinates different endocytic mechanisms. Plos Biology, 2(9), 1363-1380. doi:ARTN e261

10.1371/journal.pbio.0020261

Schroeder, H. W., Mitchell, C., Shuman, H., Holzbaur, E. L. F., \& Goldman, Y. E. (2010). Motor Number Controls Cargo Switching at Actin-Microtubule Intersections In Vitro. Current Biology, 20(8), 687-696. doi:10.1016/j.cub.2010.03.024 
Scott, C. C., Dobson, W., Botelho, R. J., Coady-Osberg, N., Chavrier, P., Knecht, D. A., . . . Grinstein, S. (2005). Phosphatidylinositol-4,5-bisphosphate hydrolysis directs actin remodeling during phagocytosis. Journal of Cell Biology, 169(1), 139-149. doi:10.1083/jcb.200412162

Smith, L. M., \& May, R. C. (2013). Mechanisms of microbial escape from phagocyte killing. Biochemical Society Transactions, 41(2), 475-490. doi:10.1042/BST20130014

Steinberg, B., Huynh, K., \& Grinstein, S. (2007). Phagosomal acidification: measurement, manipulation and functional consequences. In: Portland Press Ltd.

Sun-Wada, G. H., Tabata, H., Kawamura, N., Aoyama, M., \& Wada, Y. (2009). Direct recruitment of $\mathrm{H}+$-ATPase from lysosomes for phagosomal acidification. Journal of Cell Science, 122(14), 2504-2513. doi:10.1242/jcs.050443

Vergne, I., Chua, J., Lee, H. H., Lucas, M., Belisle, J., \& Deretic, V. (2005). Mechanism of phagolysosome biogenesis block by viable Mycobacterium tuberculosis. Proceedings of the National Academy of Sciences of the United States of America, 102(11), 4033-4038. doi:10.1073/pnas.0409716102

Vieira, O. V., Bucci, C., Harrison, R. E., Trimble, W. S., Lanzetti, L., Gruenberg, J., . . Grinstein, S. (2003). Modulation of Rab5 and Rab7 recruitment to phagosomes by phosphatidylinositol 3-kinase. Molecular and Cellular Biology, 23(7), 2501-2514. doi:10.1128/Mcb.23.7.2501-2514.2003

Wells, A. L., Lin, A. W., Chen, L. Q., Safer, D., Cain, S. M., Hasson, T., . . Sweeney, H. L. (1999). Myosin VI is an actin-based motor that moves backwards. Nature, 401(6752), 505-508. doi:Doi $10.1038 / 46835$

Yates, R. M., Hermetter, A., \& Russell, D. G. (2005). The kinetics of phagosome maturation as a function of phagosome/lysosome fusion and acquisition of hydrolytic activity. Traffic, 6(5), 413-420. doi:10.1111/j.1600-0854.2005.00284.x

Yeung, T., Heit, B., Dubuisson, J.-F., Fairn, G. D., Chiu, B., Inman, R., . . Grinstein, S. (2009). Contribution of phosphatidylserine to membrane surface charge and protein targeting during phagosome maturation. Journal of Cell Biology, 185(5), 917-928.

Yu, Y. (2021). Data: Transport Motility of Phagosomes on Actin and Microtubules Regulates Maturation Kinetics via Distinct Mechanisms. Figshare. https://figshare.com/s/4389edc40fcad49c6bbf

Yu, Y. Q., Gao, Y., \& Yu, Y. (2018). "Waltz" of Cell Membrane-Coated Nanoparticles on Lipid Bilayers: Tracking Single Particle Rotation in Ligand-Receptor Binding. Acs Nano, 12(12), 11871-11880. doi:10.1021/acsnano.8b04880

Zahn, C., Keller, S., Toro-Nahuelpan, M., Dorscht, P., Gross, W., Laumann, M., . . . Kress, H. (2017). Measurement of the magnetic moment of single Magnetospirillum gryphiswaldense cells by magnetic tweezers. Scientific Reports, 7(1), 3558. doi:10.1038/s41598-01703756-z 
Figures and figure supplements

\section{Transport Motility of Phagosomes on Actin and Microtubules Regulates Timing and Kinetics of Their Maturation}

Yanqi Yu ${ }^{a, 1}$, Zihan Zhang ${ }^{a, 1}$, Glenn F. W. Walpole ${ }^{b, c}$, and Yan $Y^{a, *}$

aDepartment of Chemistry, Indiana University, Bloomington, IN 47405-7102

bProgram in Cell Biology, The Hospital for Sick Children, Toronto, Ontario, Canada

'Department of Biochemistry, University of Toronto, Ontario, Canada 
A

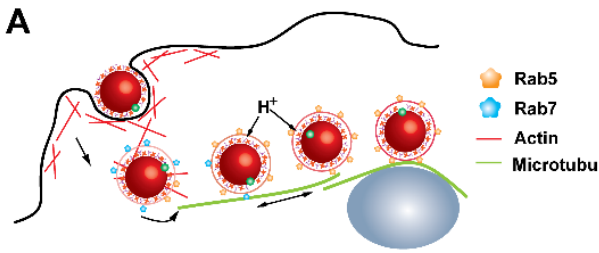

B

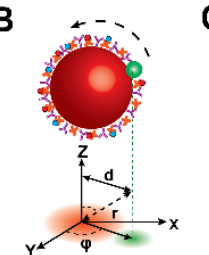

SAv-pHrodo Red

SAv-CF 640R

$Y \lg G$
C

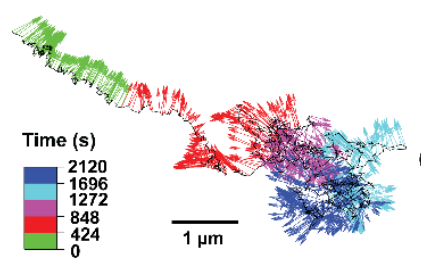

D

RotSensor

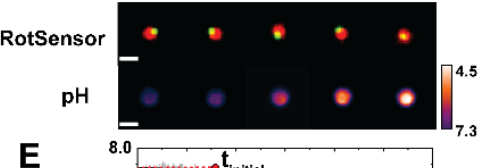

E

$\mathbf{F}$

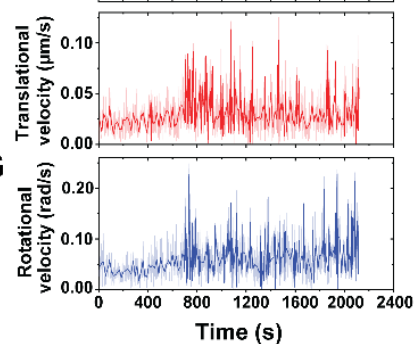

H

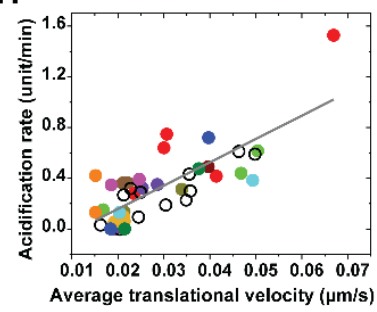

I

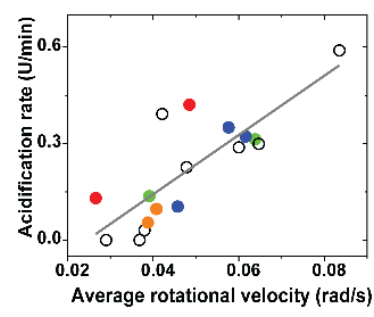

Figure 1. Simultaneous measurement of phagosome transport dynamics and acidification. (A) Schematic illustration of the experimental design. The $\mathrm{pH}$-sensitive rotational particle sensors (RotSensors) were internalized into phagosomes in activated RAW264.7 macrophage cells. (B) Schematic illustration of the design of RotSensors and the principle of single-particle rotational tracking. Each RotSensor contains a $1 \mu \mathrm{m}$ silica particles tethered with a $100 \mathrm{~nm}$ fluorescent particle. The $1 \mu \mathrm{m}$ silica particles were coated with streptavidin (SAv)-pHrodo Red (pH reporter dye) and SAv-CF640R (reference dye). The RotSensors were opsonized with physically adsorbed immunoglobulin G. The azimuthal $(\varphi)$ and polar $(\theta)$ angles of each RotSensor were analyzed based on its projection fluorescence image. In the schematic, $d$ indicates the projection inter-particle distance, and $r$ indicates the physical inter-particle distance. (C) The trajectory of a representative RotSensor-containing phagosome in a cell. Black line indicates the translational movement of the centroid of the phagosome. Each vector indicates the orientation of the RotSensor pointing from the centroid of the $1 \mu \mathrm{m}$ particle to that of the $100 \mathrm{~nm}$ particle. Vectors are color-coded based on time. Scale bar, $2 \mu \mathrm{m}$. (D-G) Fluorescence images and line curves showing acidification and transport dynamics of a representative RotSensor-containing phagosome in a cell. In the top row of fluorescence images in $D$, the pHrodoRed-functionalized 1 $\mu \mathrm{m}$ particle and its tethered $100 \mathrm{~nm}$ yellow-green particle are shown in red and green, respectively. Fluorescence images in the bottom row in $D$ show the pHrodoRed-functionalized 1 $\mu \mathrm{m}$ particle color-coded based on $\mathrm{pH}$. Scale bar, $1 \mu \mathrm{m}$. The phagosome $\mathrm{pH}$ vs. time plot $(\mathrm{E})$ is fitted with sigmoidal-Boltzmann function (shown as the black solid line) to determine the initial $\mathrm{pH}$, final $\mathrm{pH}$, the period of rapid acidification $\left(\mathrm{t}_{\text {initial }}\right.$ to $\left.\mathrm{t}_{\text {final }}\right)$, and acidification rate, as indicated by the red dotted line. (F-G) Plots showing the translational and rotational velocities of the phagosome against time, respectively. Darker lines are velocities values after wavelet denoising. (H-I) Scatter plots showing acidification rates against translational and rotational velocities of single phagosomes during rapid acidification period (from $t_{\text {initial }}$ to $t_{\text {final }}$ ). Each data point represents a single phagosome data. Data points from multiple phagosomes within the same cells are shown in the same solid color. Data points from cells containing only one phagosome are shown as black circles. Correlation between the phagosome acidification rate and transport velocities is indicated by the linear regression with a Pearson's coefficient of 0.78 in $(\mathbf{H})$ and 0.81 in (I). 
bioRxiv preprint doi: https://doi.org/10.1101/2021.04.04.438376; this version posted April 4, 2021. The copyright holder for this preprint (which was not certified by peer review) is the author/funder, who has granted bioRxiv a license to display the preprint in perpetuity. It is made available under aCC-BY-NC-ND 4.0 International license.

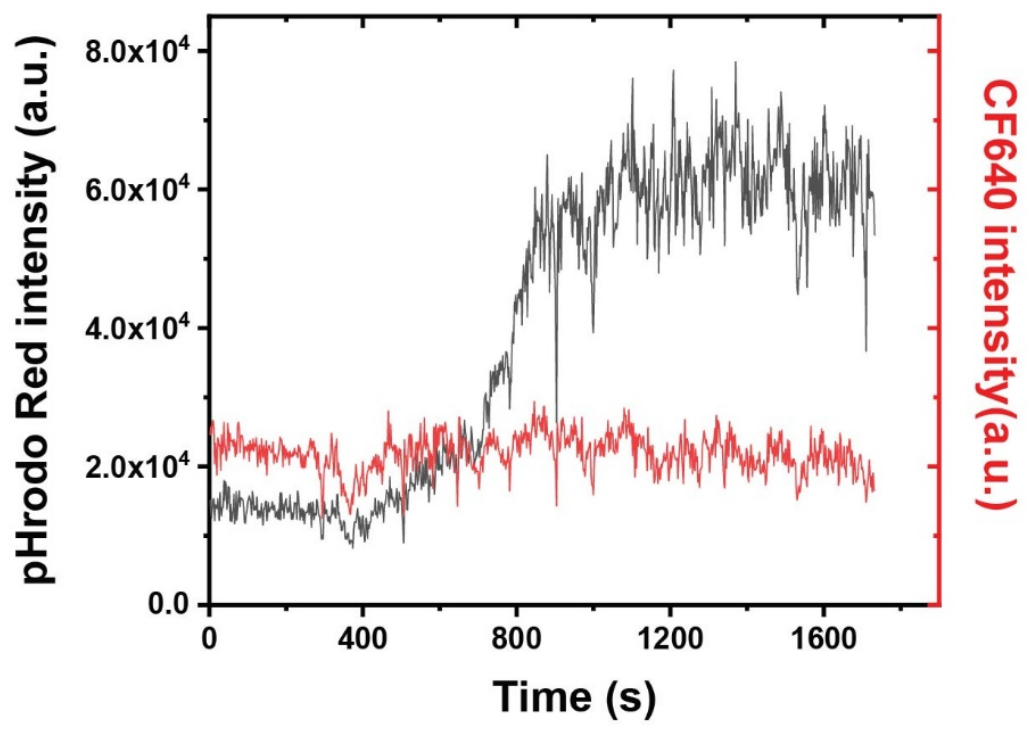

Figure 1-figure supplement 1 . Line plots showing the fluorescence emission intensity vs. time of pHrodo Red and CF640R on a representative RotSensor during phagosome acidification. 


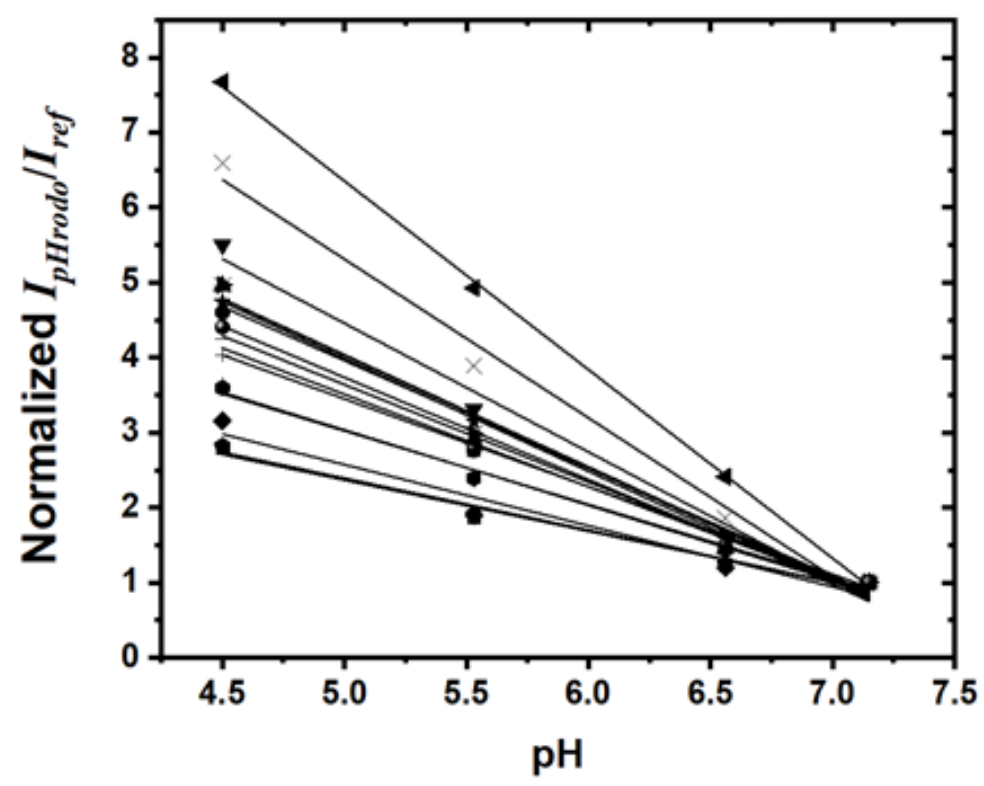

Figure 1-figure supplement 2. Representative intracellular $\mathrm{pH}$ calibration curves for 16 RotSensors in phagosomes. The fluorescence emission ratio $I_{p H r o d o} / I_{r e f}$ at each $\mathrm{pH}$ was normalized to that at $\mathrm{pH}$ 7.3. Black lines indicate linear fittings of the data. An average $\mathrm{R}^{2}$ value of $0.98 \pm 0.02$ was obtained from the individual linear fittings. 


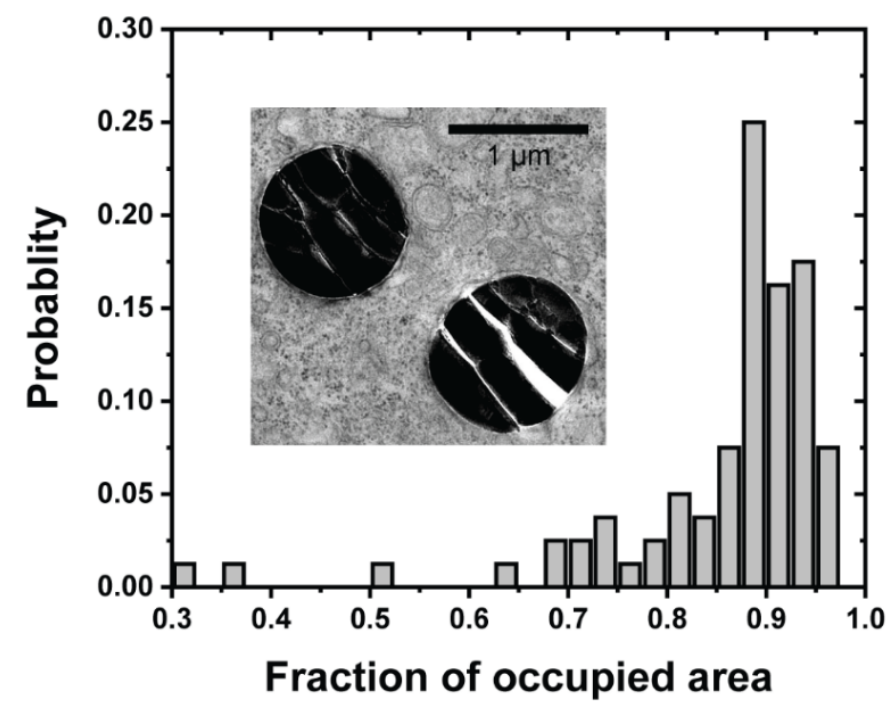

Figure 1-figure supplement 3. Characterization of the tightness of phagosome membrane wrapping around internalized particle sensors. Inset is a representative TEM image showing particle sensors encapsulated inside phagosomes. Fraction of occupied area is defined as the ratio of the area occupied by the particle sensor to the total area of the phagosome in each given TEM section. 
A

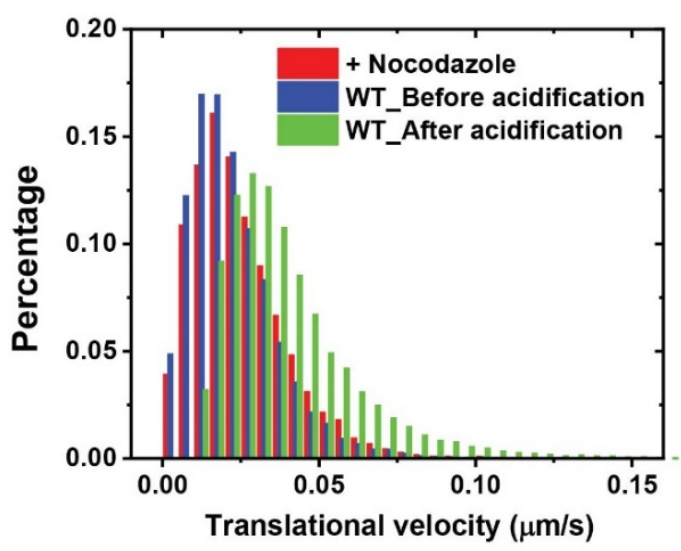

B

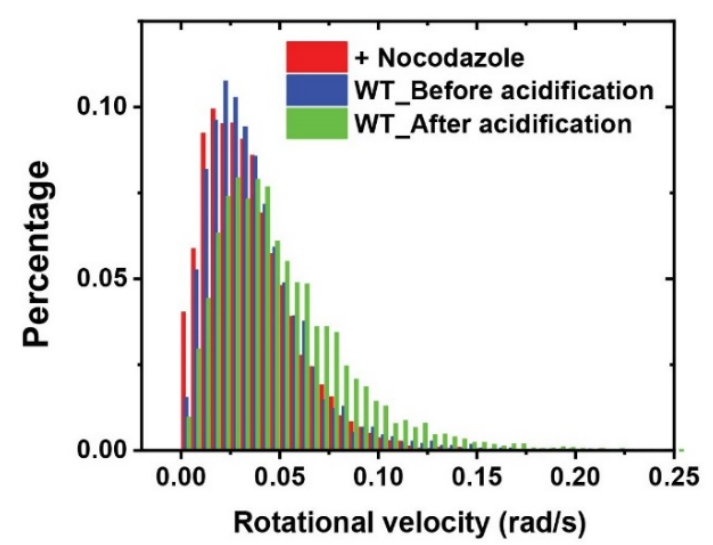

Figure 1-figure supplement 4. Histograms showing the distribution of $(\mathbf{A})$ translational and (B) rotational velocities of phagosomes in control cells (WT) and cells treated with nocodazole, a microtubule-depolymerizing reagent. Data were obtained from 8 individual phagosomes from nocodazole-treated cells and 30 phagosomes from control cells. 
A

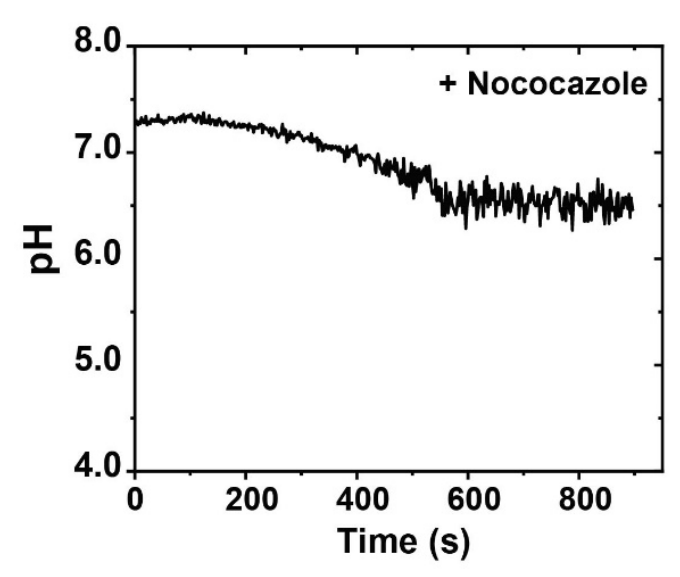

B

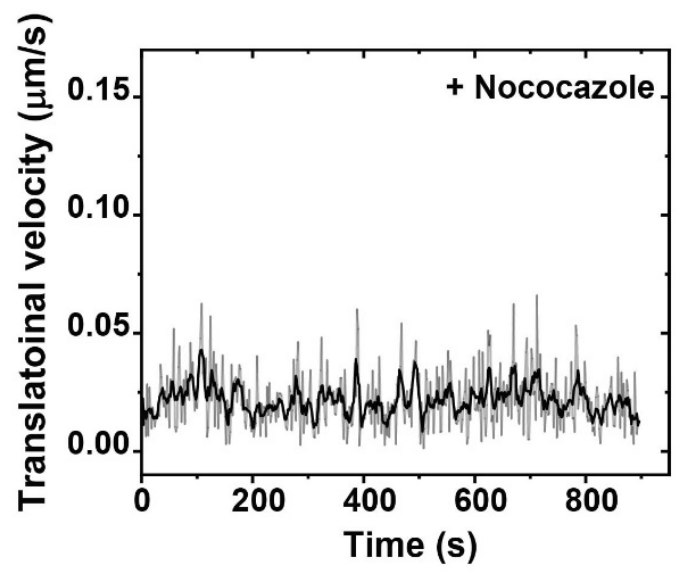

Figure 1-figure supplement $\mathbf{5}$. The acidification (A) and translational velocity (B) of a representative phagosome in nocodazole- treated cell. Thick line in (B) indicates data after wavelet denoising. 

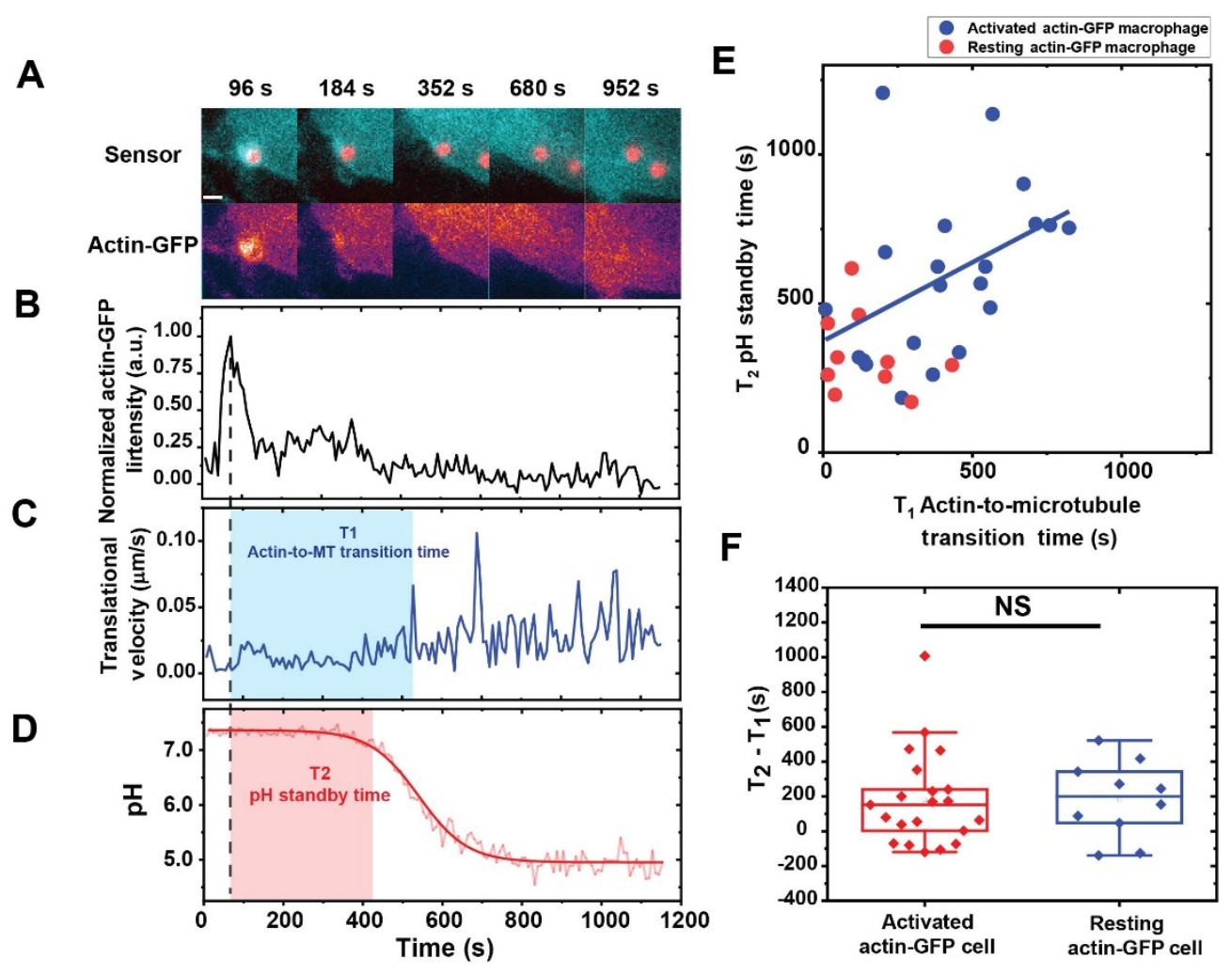

Figure 1-figure supplement 6 . Phagosome dynamics and acidification in resting and activated actin-GFP macrophage cells. (A) Fluorescent images showing subcellular locations of RotSensorcontaining phagosomes at different time points in actin-GFP expressing cells. Scale bar, $2 \mu \mathrm{m}$. (BD) Plots showing the normalized actin-GFP intensity (B), translational velocity (C), and $\mathrm{pH}(\mathbf{D})$ of the phagosome shown in (A) as a function of time. In (C), the blue shade indicates the actin-tomicrotubule transition period $\left(T_{1}\right)$. In (D), the thick red line indicates $\mathrm{pH}$ after sigmoidal-Boltzmann fitting. The red shade indicates $\mathrm{pH}$ standby time $\left(\mathrm{T}_{2}\right)$. (E) Scatter plot showing actin-to-microtubule transition time against $\mathrm{pH}$ standby time of single phagosomes in the activated and resting actinGFP macrophage cells. The thick blue line indicates a linear fit to the data points from activated cells, which has a Pearson's coefficient of 0.44. (F) Box plots showing the time difference between actin-to-microtubule transition time and $\mathrm{pH}$ standby time $\left(\mathrm{T}_{2}-\mathrm{T}_{1}\right)$ for activated and resting actin-GFP macrophage cells. Each box plot indicates the mean (horizontal line) and the interquartile range from $25 \%$ to $75 \%$ of the corresponding data set. NS represents not significant. 
A

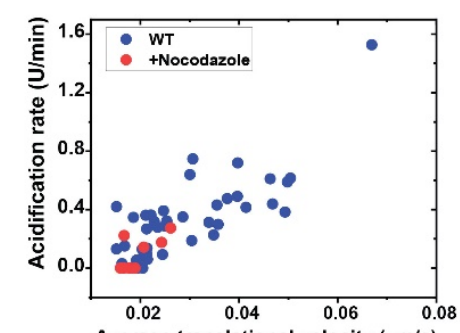

C

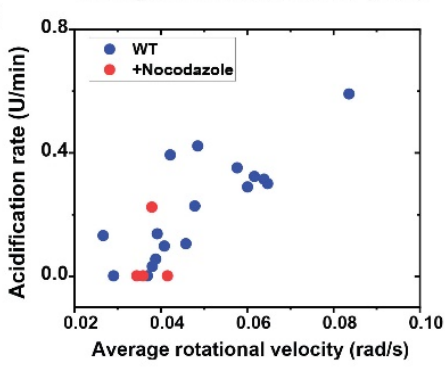

B

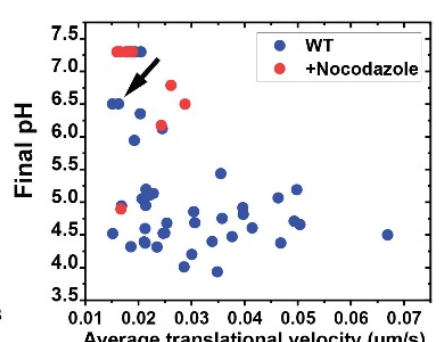

D

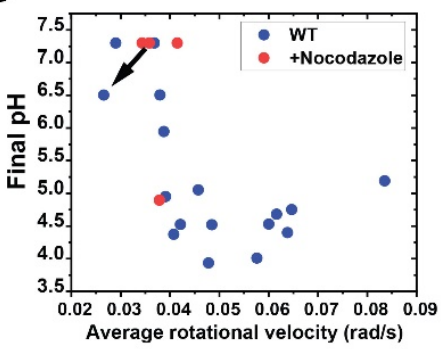

E
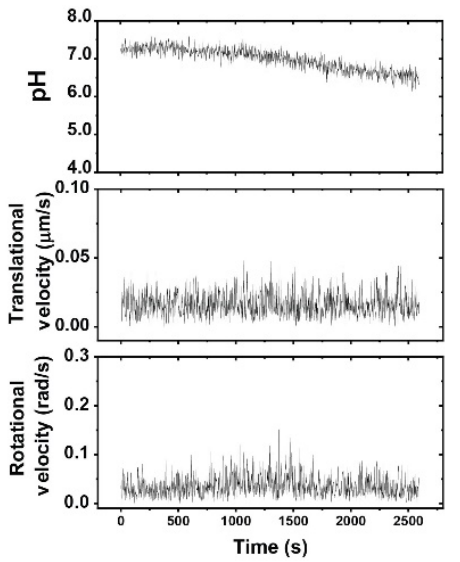

Figure 1-figure supplement 7. (A-D) Scatter plots showing acidification rate and final $\mathrm{pH}$ of single phagosomes plotted separately against their translational and rotational velocity during rapid acidification period in control cells (WT) and cells treated with nocodazole. (E) Acidification, translational velocity and rotational velocity profiles of a slow-moving phagosome indicated by the arrows in (B) and (D). 


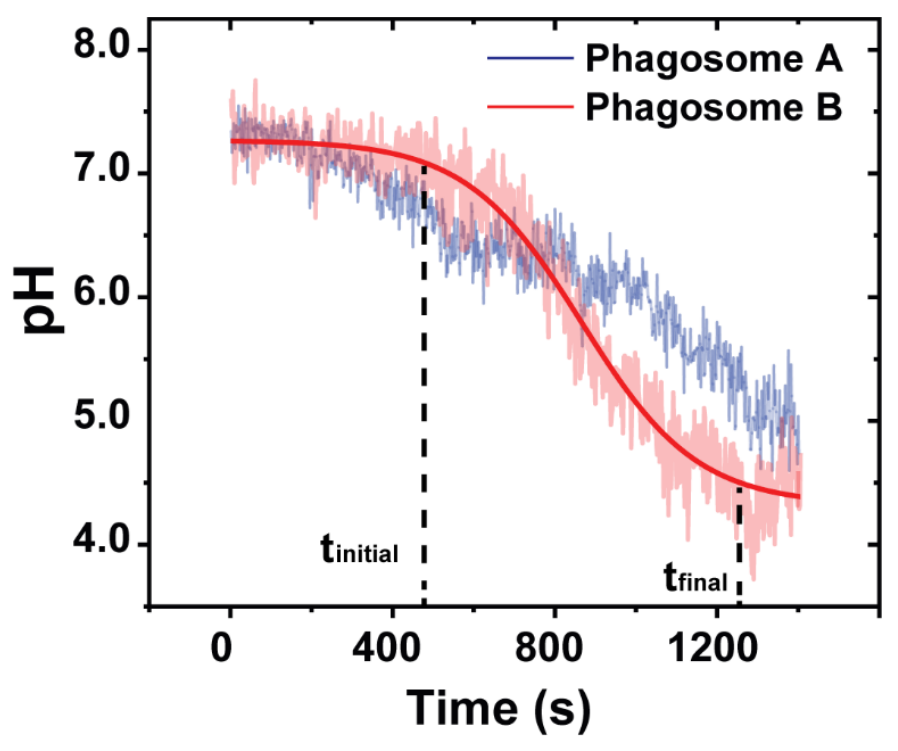

Figure 1-figure supplement 8 . Acidification profiles of two phagosomes from a same cell. The $\mathrm{pH}$-time plot of phagosome $\mathrm{B}$ is fitted with sigmoidal-Boltzmann function (shown as thick red line) to determine the acidification rate, the beginning $\left(\mathrm{t}_{\text {initial }}\right)$ and the end $\left(\mathrm{t}_{\text {final }}\right)$ of the rapid acidification. 


\section{A}

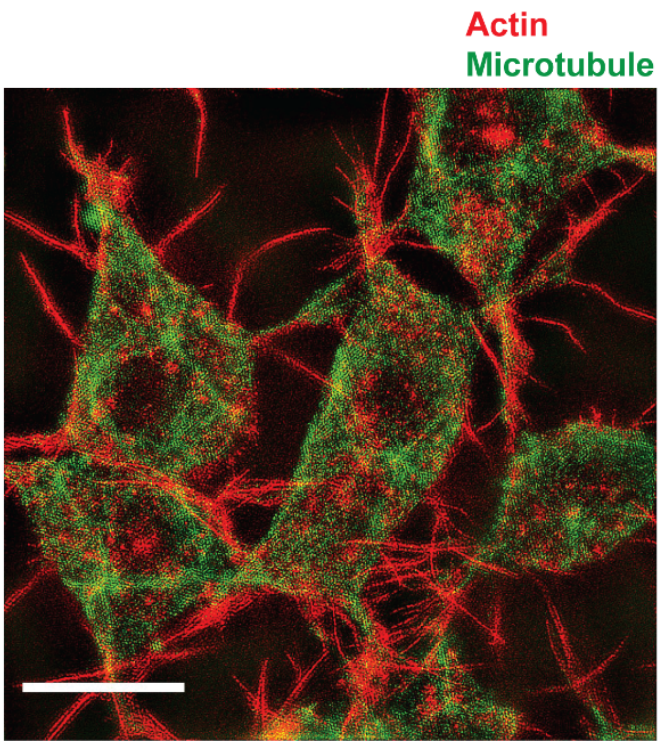

B

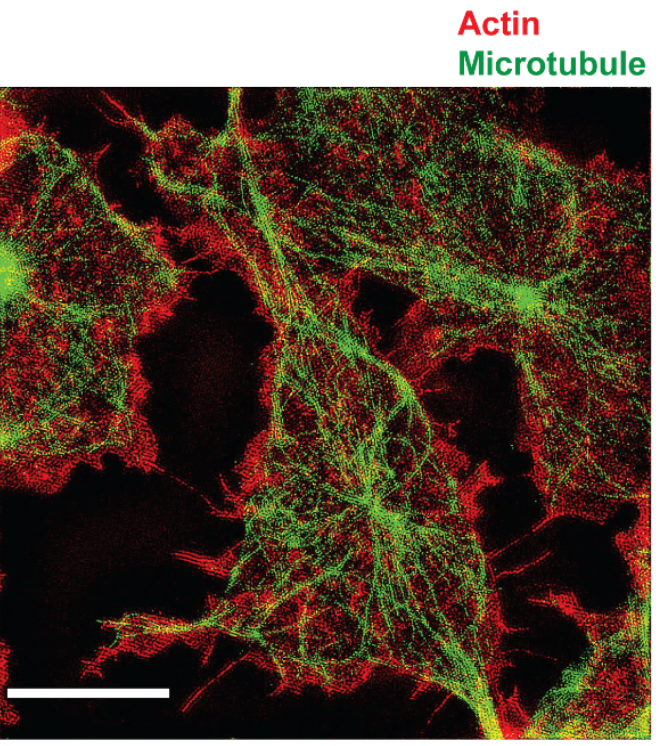

Figure 1-figure supplement 9. Structured illumination microscopy (SIM) images showing immunostained actin (shown in red) and microtubules (green) in resting (A) and activated (B) macrophages. Scale bars, $10 \mu \mathrm{m}$. 


\section{A}

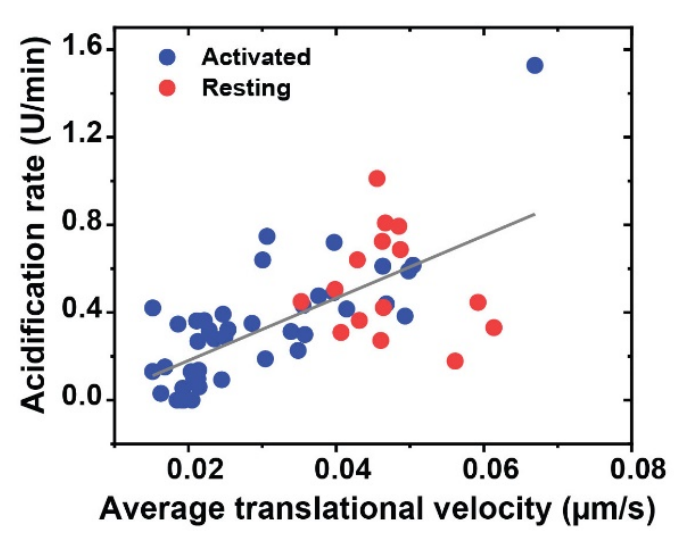

B

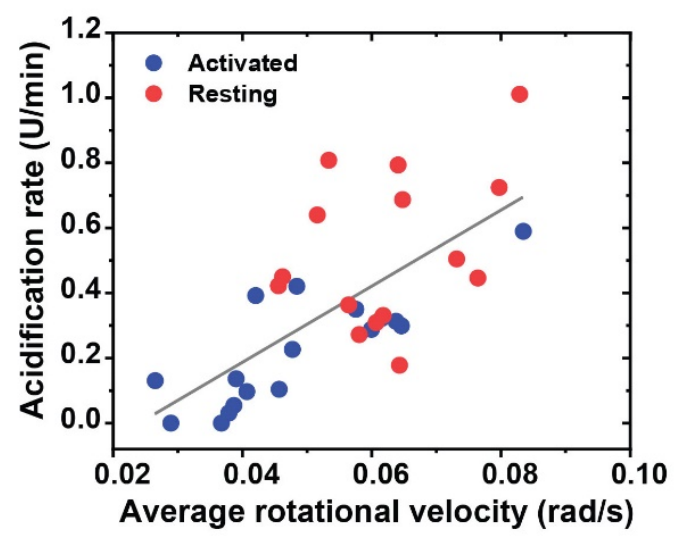

Figure 1-figure supplement 10 . Scatter plots showing acidification rates against average translational $(\mathbf{A})$ and rotational $(\mathbf{B})$ velocities of single phagosomes during their rapid acidification period in activated and resting macrophages. Black lines indicate linear fitting to the data. Pearson coefficients are 0.68 and 0.69 for the fitting in (A) and (B), respectively. 
bioRxiv preprint doi: https://doi.org/10.1101/2021.04.04.438376; this version posted April 4, 2021. The copyright holder for this preprint (which was not certified by peer review) is the author/funder, who has granted bioRxiv a license to display the preprint in perpetuity. It is made available under aCC-BY-NC-ND 4.0 International license.

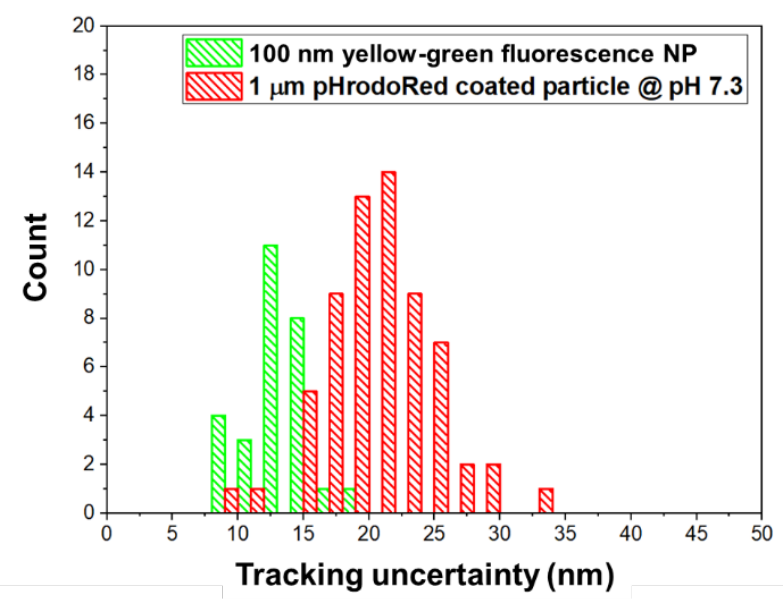

Figure 1-figure supplement 11 . Histograms showing localization uncertainties for tracking the two particle components in a single RotSensor: the $100 \mathrm{~nm}$ yellow-green nanoparticle and the 1 $\mu \mathrm{m}$ pHrodoRed-coated particle. 
A

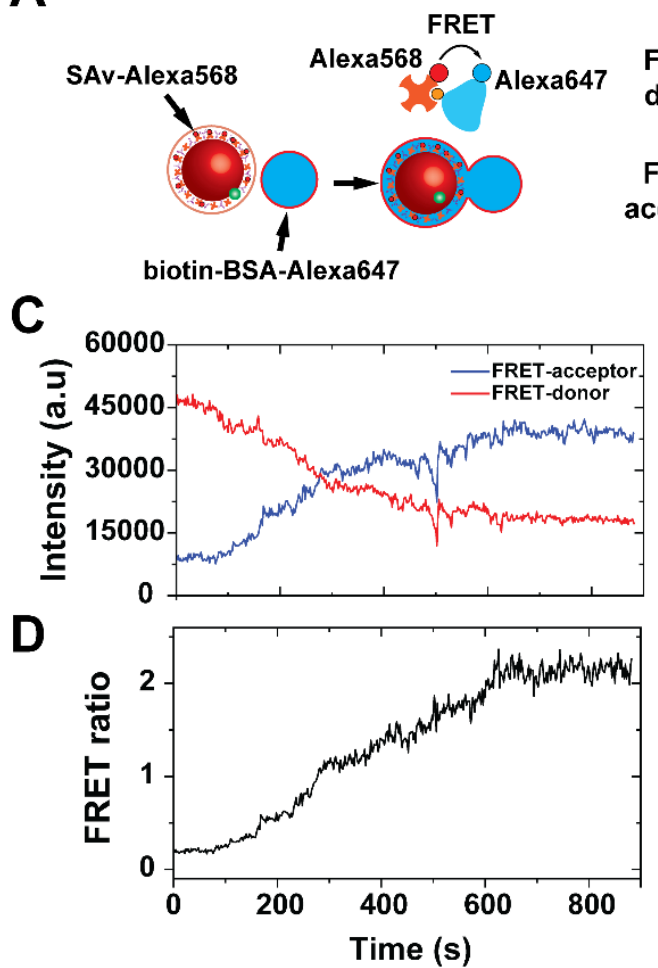

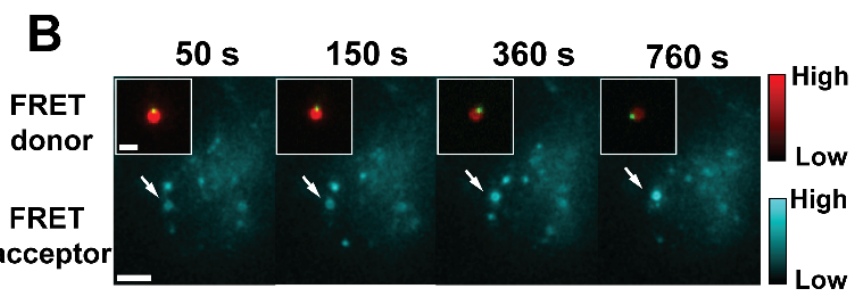

E $\overline{\underline{\varepsilon}}$

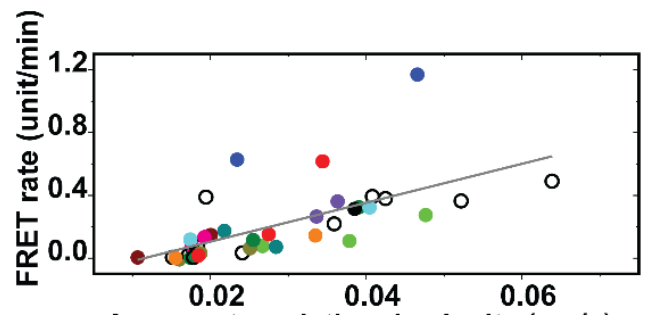

$\mathbf{F}$

Average translational velocity $(\mu \mathrm{m} / \mathrm{s})$

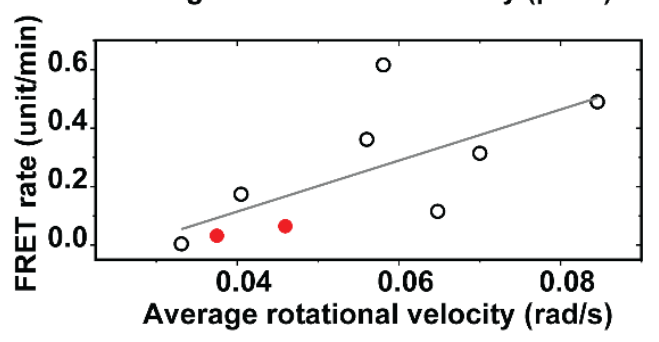

Figure 2. Simultaneous measurement of phagosome-lysosome fusion and phagosome transport dynamics. (A) Schematic illustration of the Förster resonance energy transfer (FRET)-based fusion assay. Streptavidin conjugated with the donor fluorophore Alexa-568 was coated on the RotSensor and BSA-biotin conjugated with the acceptor fluorophore Alexa-647 was encapsulated in lysosomes. Phagosome fusion with lysosomes leads to binding between streptavidin and BSAbiotin, which induces FRET between Alexa-568 and Alexa-647. (B-D) Fluorescence images and plots showing the FRET signal change of a representative phagosome during maturation in an activated RAW264.7 macrophage cell. The FRET ratio is defined as the ratio of donor (Alexa568) and acceptor (Alexa647) fluorescence emission intensity under donor excitation. Scale bar, $5 \mu \mathrm{m}$. Scale bar in inset, $2 \mu \mathrm{m}$. (E-F) Scatter plots showing FRET rates against translational and rotational velocities of single phagosomes during phagosome-lysosome fusion period. Each data point represents a single phagosome data. Data points from multiple phagosomes within the same cells are shown in the same solid color. Data points from cells containing only one phagosome are shown as black circles. The black lines indicate linear regression with a Pearson's coefficient of 0.65 in $(\mathbf{E})$ and of 0.68 in $(\mathbf{F})$. 
bioRxiv preprint doi: https://doi.org/10.1101/2021.04.04 438376; this version posted April 4, 2021. The copyright holder for this preprint (which was not certified by peer review) is the author/funder, who has granted bioRxiv a license to display the preprint in perpetuity. It is made available under aCC-BY-NC-ND 4.0 International license.

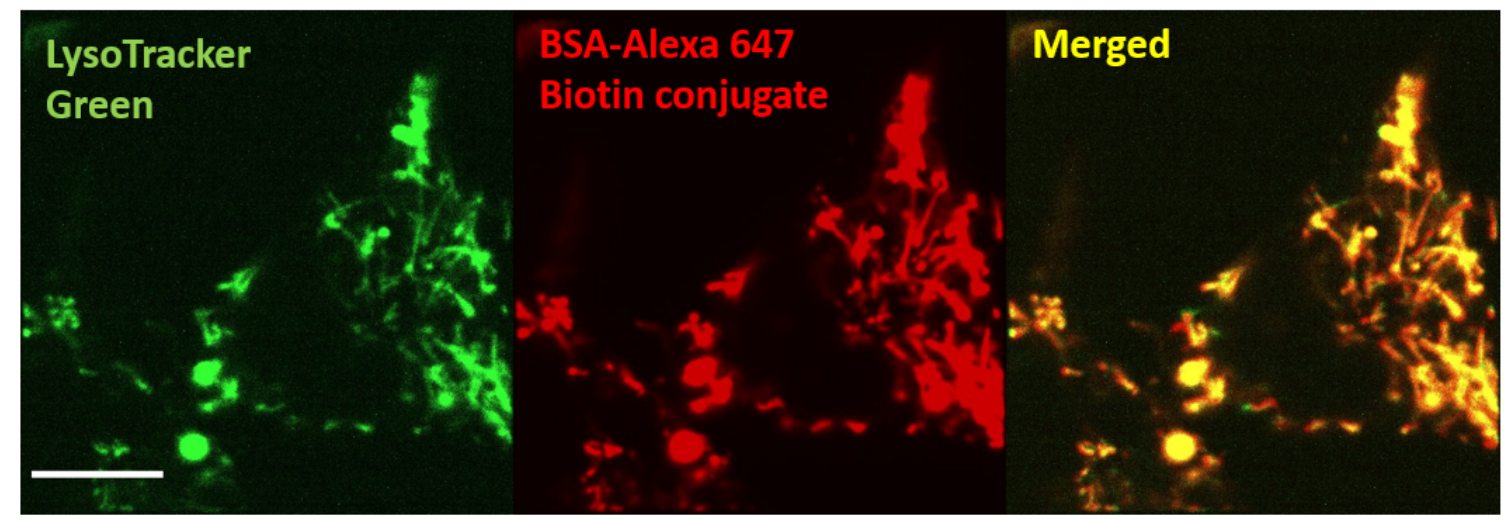

Figure 2-figure supplement 1. Re-scan confocal microscopy (RCM) images showing the colocalization of Lysotracker Green with intracellular organelles containing BSA-Alexa647 in activated RAW 264.7 macrophage cells. Scale bar, 10 $\mu \mathrm{m}$. 
A

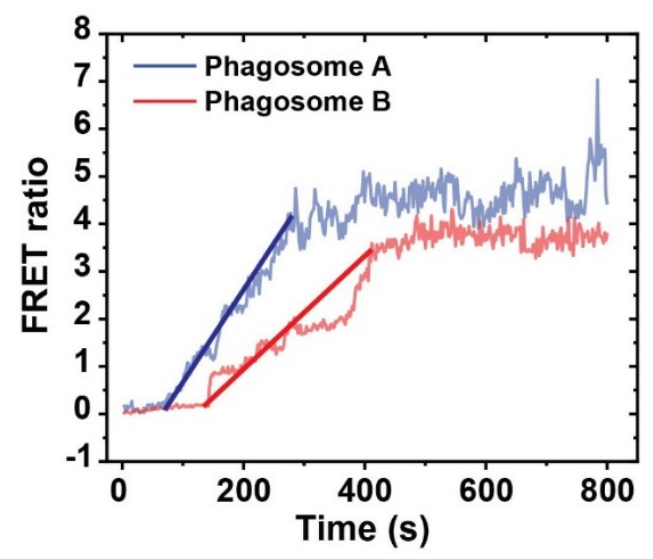

B

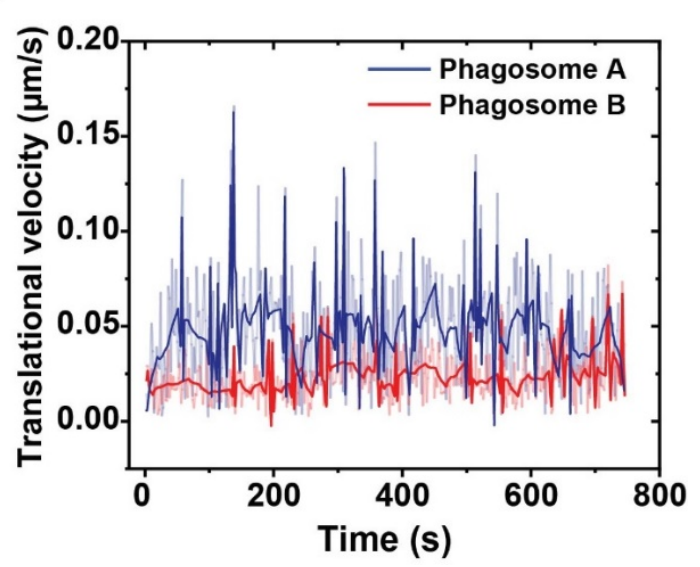

Figure 2-figure supplement 2. Plots showing (A) FRET ratio vs. time and (B) translational velocity of two phagosomes within a single cell. Slope of the linear portion of each plot in (A), as indicated by the thick blue and red lines, was obtained as FRET rate. Dark lines in (B) are velocities values after wavelet denoising. 
A

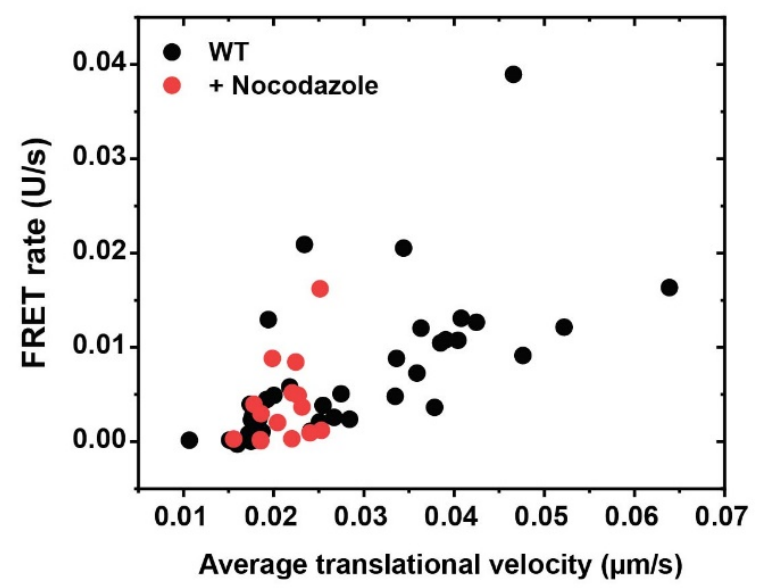

B

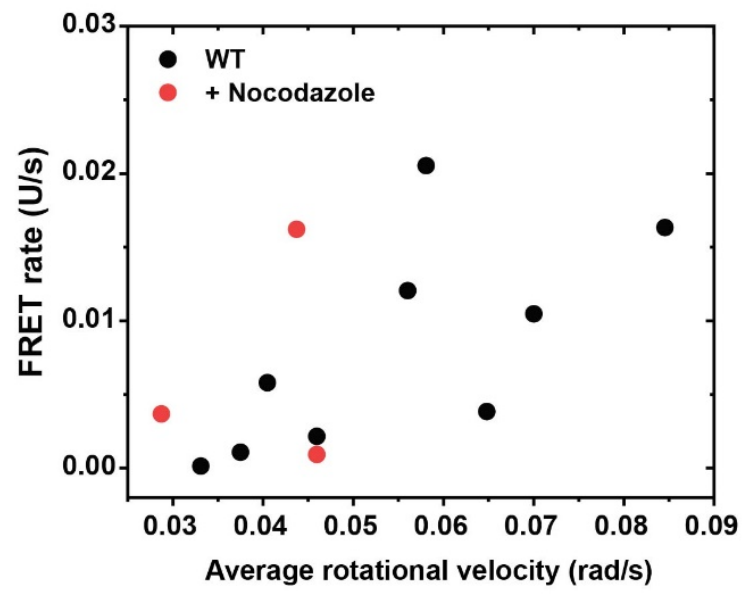

Figure 2-figure supplement 3. Scatter plots showing FRET rate against the average translational $(\mathbf{A})$ and rotational $(\mathbf{B})$ velocities of single phagosomes during phagosome-lysosome fusion in cells with or without nocodazole treatment. 
A

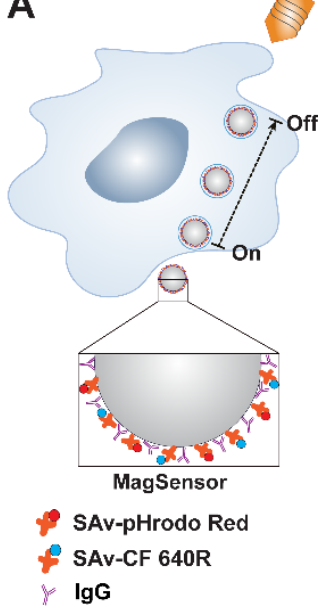

B

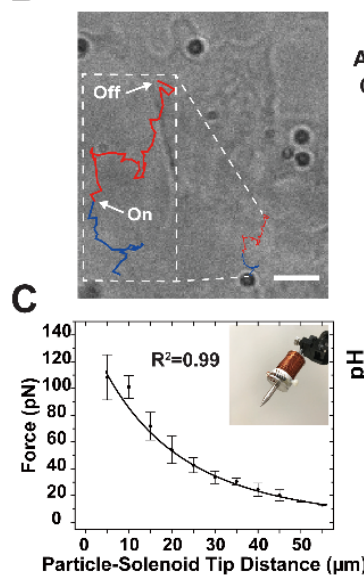

D

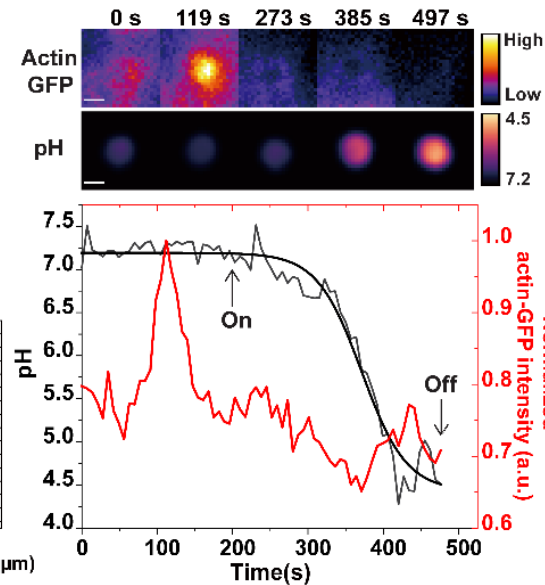

E
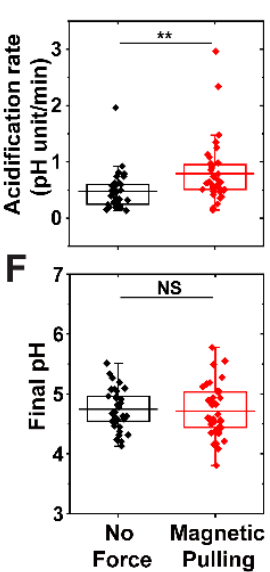

Figure 3. Phagosome acidification during magnetically manipulated transport. (A) Schematic illustration of the experimental design. Magnetic pulling force was applied on MagSensors after internalization in resting RAW 264.7 macrophage cells. The $1 \mu \mathrm{m}$ MagSensors were coated with pHrodo Red-SAv (pH indicator), CF640-SAv (reference), and physically adsorbed IgG. (B) Brightfield image of a representative cell overlaid with the trajectory of a MagSensor-containing phagosome under magnetic pulling. The start and end time points of magnetic force are indicated. Scale bar, $5 \mu \mathrm{m}$. (C) Calibration plot showing the magnetic force exerted on the MagSensors as a function of the distance from the MagSensor to the tip of the magnetic tweezers solenoid (shown in inset). Error bars are standard deviation from 5 samples. (D) Fluorescence images and line curves showing changes of $\mathrm{pH}$ and actin intensity of a representative phagosome during acidification that was magnetically pulled. Fluorescence images showing the phagosome $\mathrm{pH}$ and actin-GFP in cells are color-coded based on the scales indicated. Scale bars, $1 \mu \mathrm{m}$. The change of phagosome $\mathrm{pH}$ and the corresponding sigmoidal-Boltzmann fitting are plotted in black lines. Data of actin-GFP intensity around the phagosomes are plotted in red lines. The "on" and "off" time points of magnetic pulling are indicated. (E) Statistic result showing the average acidification rate of phagosomes with or without magnetic pulling. The average acidification rate is $0.48 \pm 0.34 \mathrm{pH}$ unit/min without magnetic manipulation $(\mathrm{N}=33)$ and $0.79 \pm$ $0.54 \mathrm{pH}$ unit/min with magnetic pulling $(\mathrm{N}=38)$. $(\mathrm{F})$ Statistic result showing the average final $\mathrm{pH}$ in different experiment conditions as indicated. The average final $\mathrm{pH}$ is $4.7 \pm 0.3$ without magnetic manipulation $(\mathrm{N}=33)$ and $4.7 \pm 0.4$ with magnetic pulling $(\mathrm{N}=38)$. In both scattered plots, each box plot indicates the mean (horizontal line) and the interquartile range from $25 \%$ to $75 \%$ of the corresponding data set. Statistical significance is highlighted by $p$-values (student's t-test) as follows: ${ }^{* *} p<0.01$, NS $p>0.05$. 
A

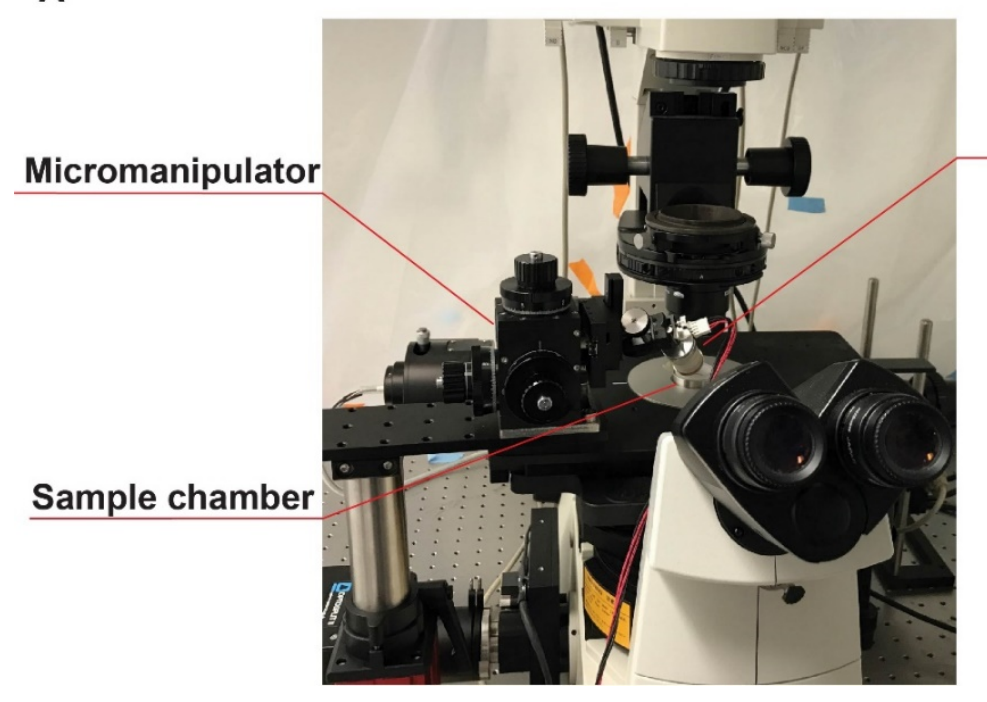

B

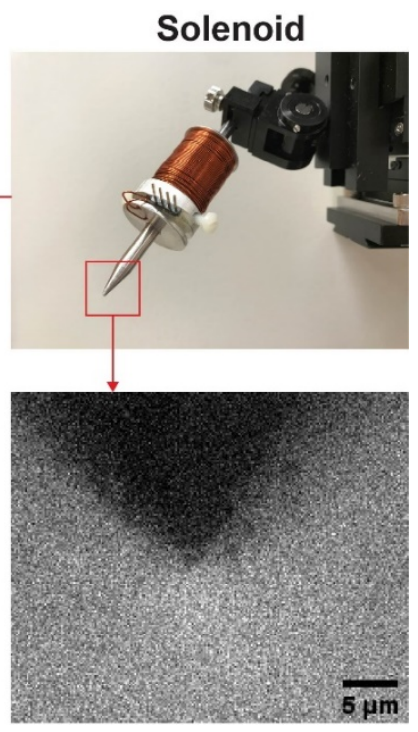

Figure 3-figure supplement 1. Magnetic tweezers setup. (A) Picture of the magnetic tweezers setup installed on an inverted Nikon microscope. (B) Picture of the solenoid. The metal core is inserted into an aluminum bobbin wrapped with copper coils. (C) Bright-field image of the solenoid tip. 

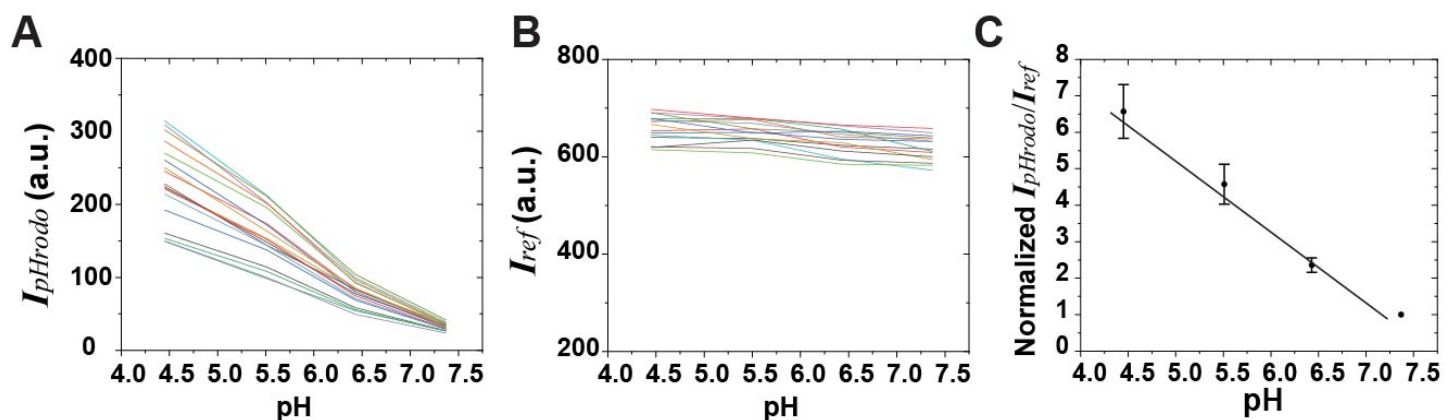

Figure 3-figure supplement 2. Extracellular $\mathrm{pH}$ calibration of MagSensors. Fluorescence intensity of $\mathrm{pH}$-indicator pHrodo Red (A) and reference dye CF640R (B) on individual MagSensors is plotted against $\mathrm{pH}$ in aqueous buffer solutions. Each curve represents data from a single MagSensor and a total of 15 particles were analyzed for each sample. (C) The fluorescence intensity ratio of pHrodo Red and CF-640R of individual MagSensors was calculated and averaged to obtain a pH calibration plot in aqueous buffer solutions. Error bars are standard deviations from 15 individual MagSensors. $R^{2}$ value of the linear fitting is 0.99 . 

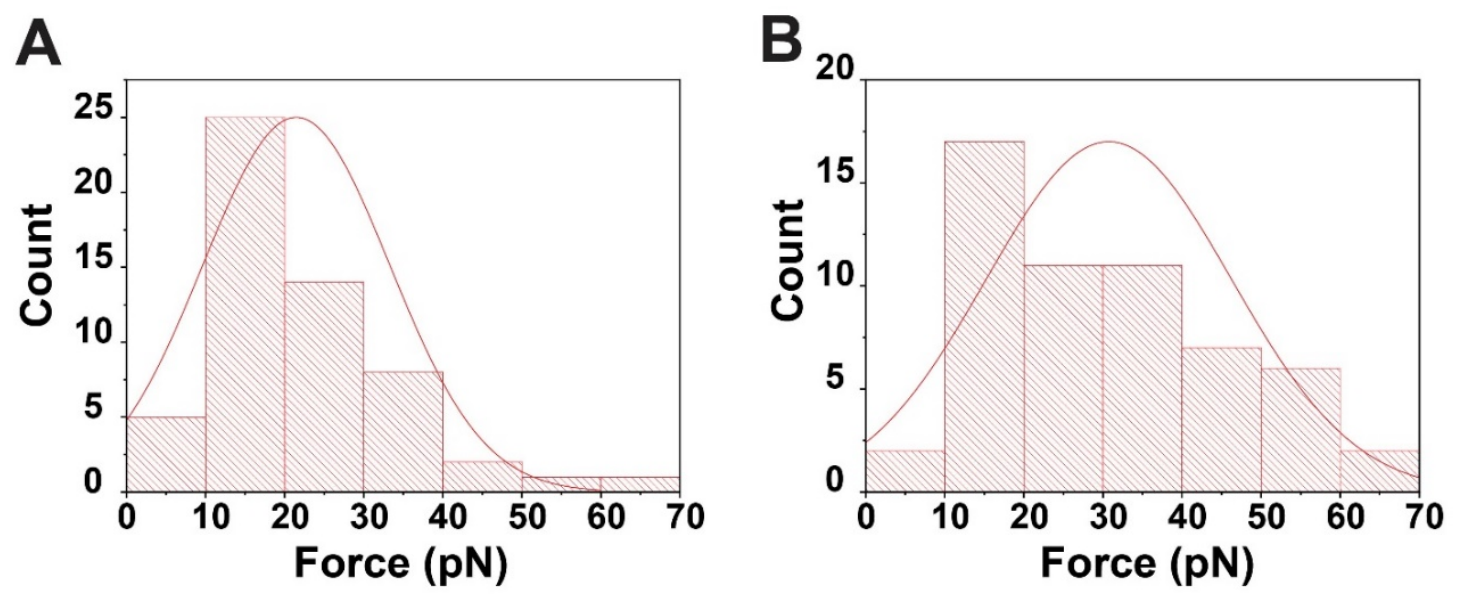

Figure 3-figure supplement 3. Distribution of estimated magnetic force exerted on individual phagosomes at the beginning $(\mathbf{A})$ and the end of experiments (B). The average magnetic force exerted at the beginning and the end of magnetic pulling was around $21 \mathrm{pN}$ and $31 \mathrm{pN}$, respectively. 


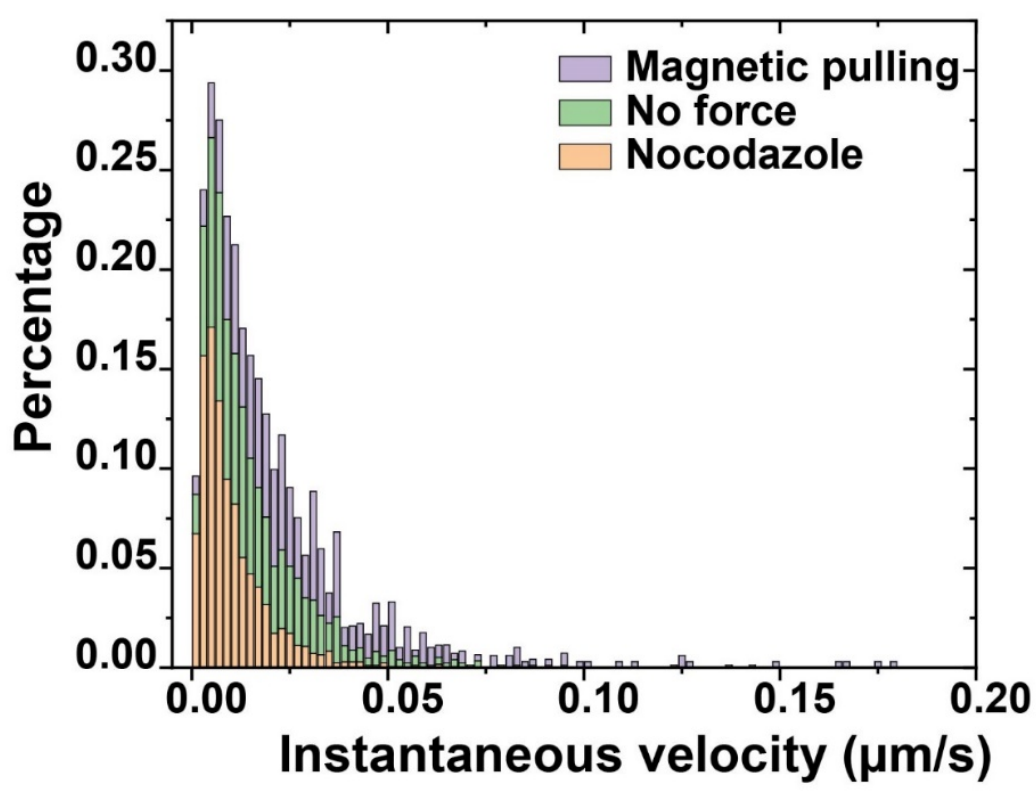

Figure 3-figure supplement 4. Histograms showing the distribution of instantaneous velocities of phagosomes in resting RAW264.7 macrophage cells under different experimental conditions: without or with magnetic pulling, and after nocodazole treatment. Each set of data includes results from 5 individual phagosomes from 5 cells. 


\section{A}
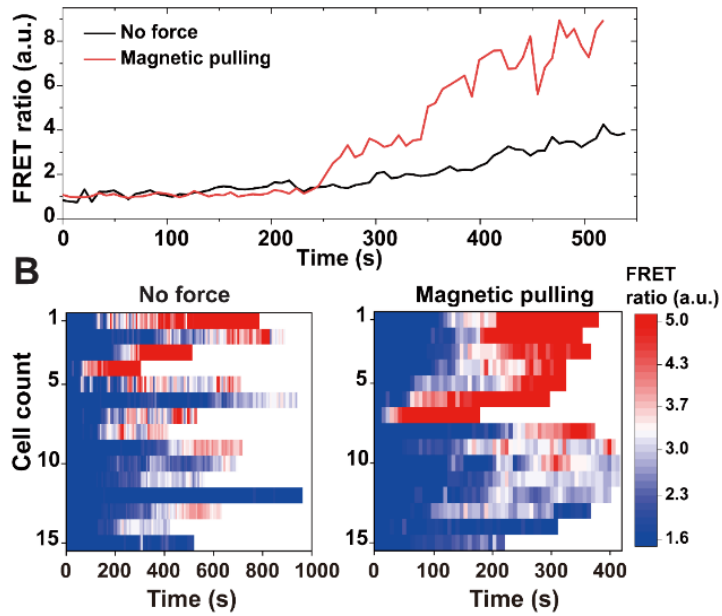

Figure 4. Phagosome-lysosome fusion during magnetically manipulated transport. (A) Line plots showing FRET ratio vs. time for two representative phagosomes with or without magnetic pulling. Time zero indicates the start of magnetic pulling force. (B) Heatmaps showing color-coded FRET ratio vs. time of all phagosomes ( $\mathrm{N}=15$ for each graph) with or without magnetic pulling. 

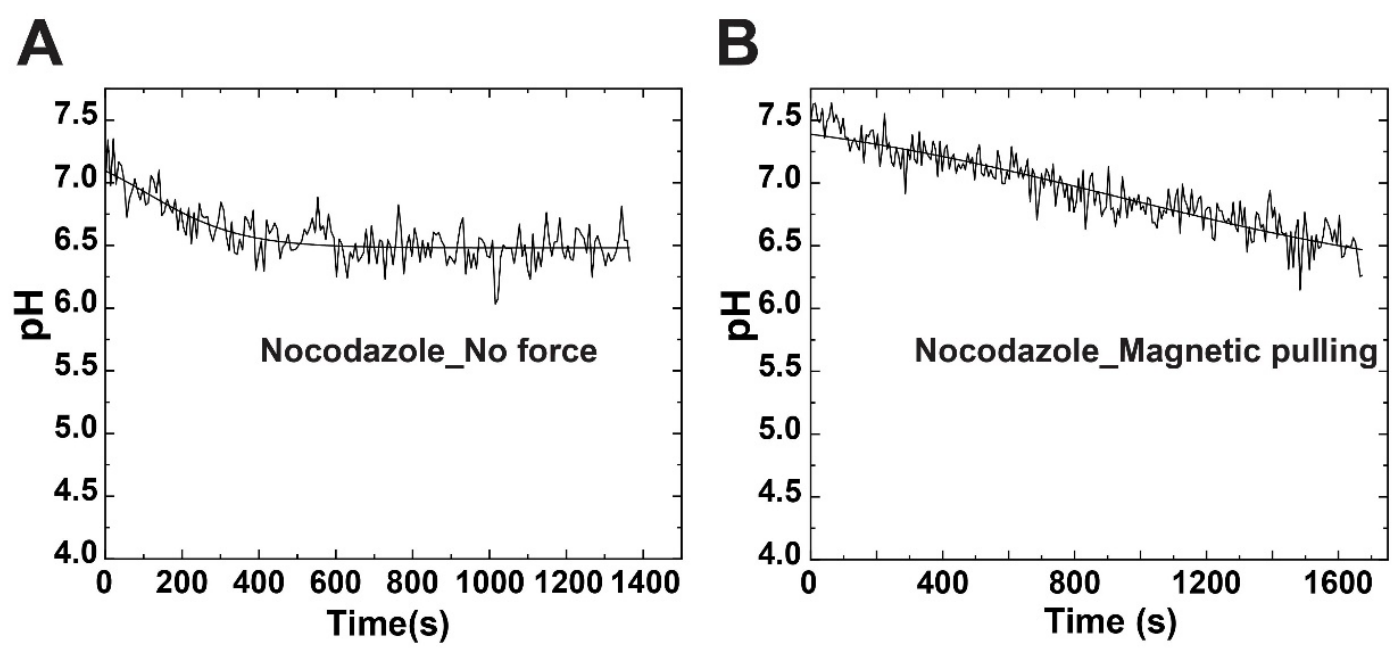

Figure 4-figure supplement 1. Acidification profile of representative single phagosomes after nocodazole treatment without $(\mathbf{A})$ or with $(\mathbf{B})$ magnetic pulling. Each $\mathrm{pH}$-time curve is fitted with sigmoidal-Boltzmann function. 


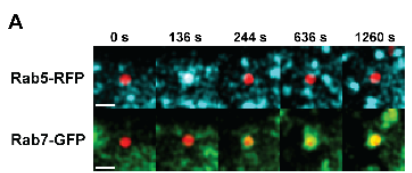

B

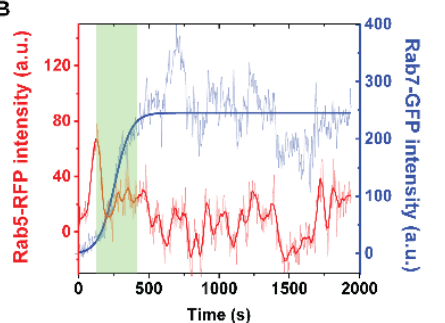

C

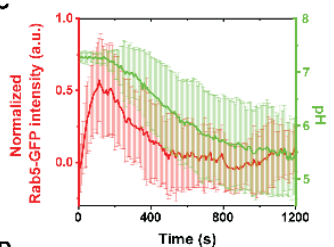

E

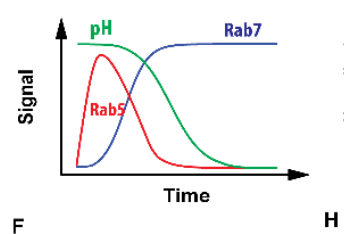

$\mathbf{G}$
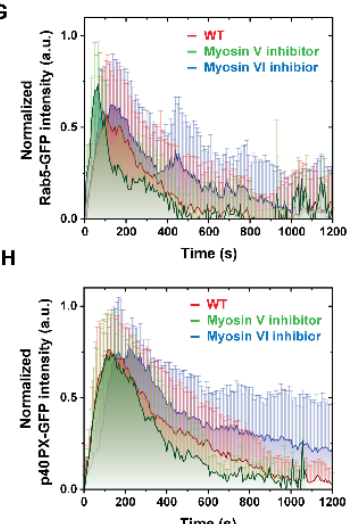

Figure 5. Dependence of early-to-late phagosome transition on actin-to-microtubule transport. (A-B) Fluorescence images and intensity plots showing the dynamic distribution of Rab5-RFP and Rab7-GFP around a representative phagosome during maturation in an activated RAW 264.7 macrophage cell. In the overlaid fluorescence images in (A), the $\mathrm{pH}$-sensor containing phagosome is shown in red, Rab5-RFP in cyan and Rab7-GFP in green. Scale bar, $2 \mu \mathrm{m}$. The thick blue line indicates sigmoidal-Boltzmann function fitting to the Rab7-GFP data. The green shaded area indicates the period of Rab5-to-Rab7 transition from the first Rab5 intensity peak to the plateau of Rab7-GFP intensity. (C-D) Line plots showing average normalized intensity of Rab5-GFP (in C), Rab7-GFP (in D), and average normalized phagosome pH (in both plots) as a function of time. The line curves were averaged from 26 and 17 individual phagosomes in Rab5RFP and Rab7-GFP expressing cells, respectively. Vertical bars indicate standard deviations. (E) A schematic showing the dynamic changes of Rab5, Rab7, and pH during singe phagosome acidification. (F) Scatter plot showing actin-to-microtubule transition time of single phagosomes with or without drug treatments as indicated. The average actin-to-microtubule transition time of phagosomes is $440 \pm 266 \mathrm{~s}$ in cells without drug treatment (WT), $276 \pm 214 \mathrm{~s}$ in cells with Myosin $\mathrm{V}$ inhibitor, and $1026 \pm 487 \mathrm{~s}$ in cells with Myosin VI inhibitor. In all scattered plots, each box plot indicates the mean (horizontal line) and the interquartile range from $25 \%$ to $75 \%$ of the corresponding data set. Statistical significance is highlighted by $p$-values (student's t-test) as follows: ${ }^{* * *} p<0.0001,{ }^{*} p<0.05$. (G) Plots showing average normalized intensity of Rab5-GFP as a function of time with or without drug treatments as indicated. The line curves were averaged from 26, 10, and 16 individual phagosomes in cells without drug treatment (WT), with Myosin V inhibitor, and with Myosin VI inhibitor, respectively. Vertical bars represent standard deviations. (H) Plots showing the average normalized intensity of p40PX-GFP, a probe for PI3P, in cells under different drug treatment. The line curves are averaged from 12, 14, and 8 individual phagosomes without drug treatment (WT), with Myosin V inhibition, and with Myosin VI inhibition, respectively. Vertical bars represent standard deviations. 


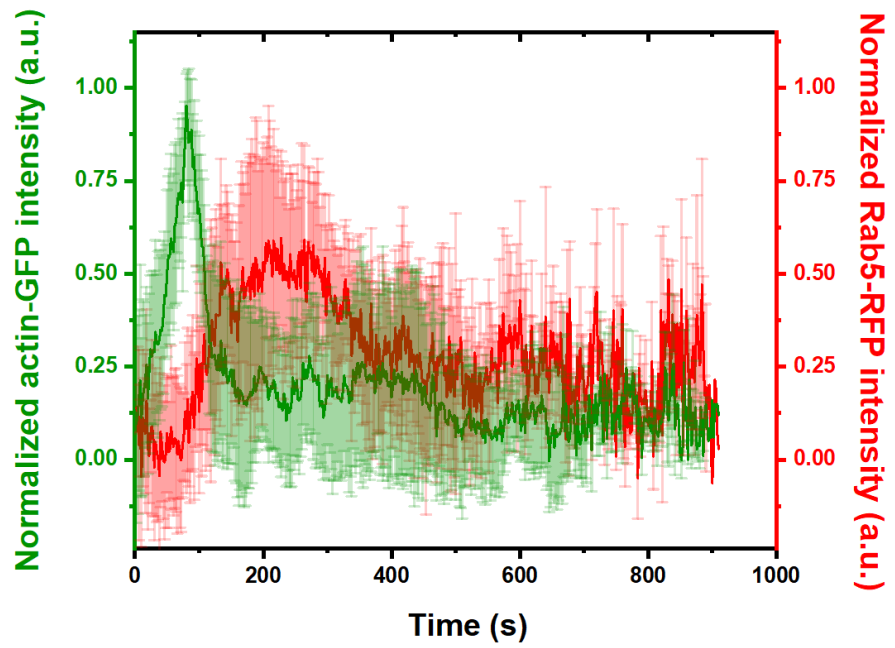

Figure 5-figure supplement 1. Data showing normalized fluorescence intensity vs. time for actinGFP and Rab5-RFP on phagosomes obtained from dual-channel simultaneous imaging. Fluorescence intensity of actin-GFP and Rab5-GFP was separately normalized based on its cytosolic background. Each plot is an average of results from 9 independent phagosomes and error bars indicate standard deviations. 

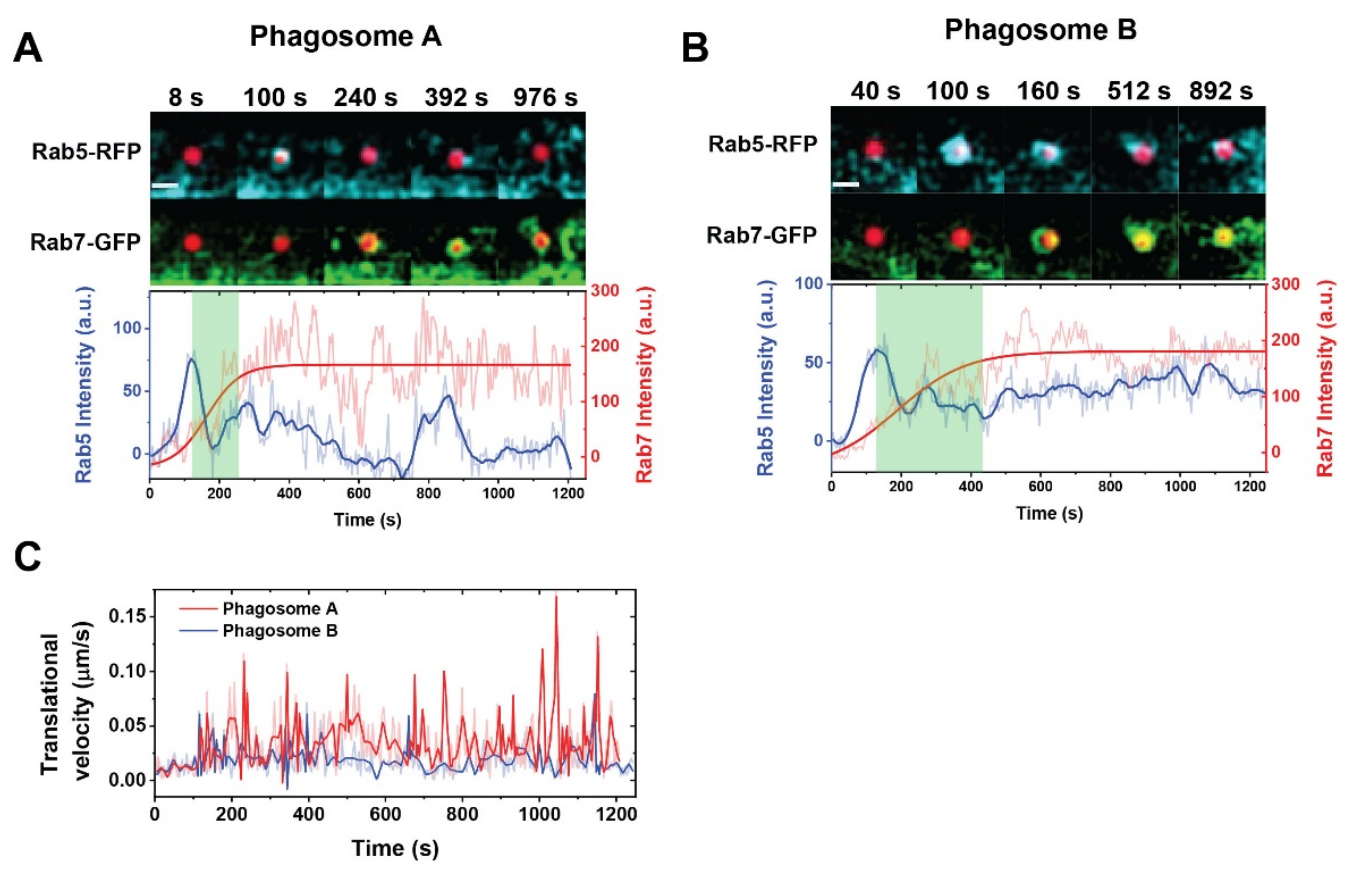

Figure 5-figure supplement 2. (A-B) Fluorescence images and intensity plots showing the dynamic distribution of Rab5-RFP and Rab7-GFP around two phagosomes within the same cell. The fluorescence images show the overlay between pH-sensor (red) with Rab5-GFP (cyan) and Rab7-GFP (green) separately. Scale bars, $2 \mu \mathrm{m}$. The green shades in the fluorescence intensity vs. time plots indicate the duration of Rab5-to-Rab7 transition that begins at the Rab5-RFP intensity peak and ends at the onset of Rab7-GFP intensity plateau determined by sigmoidal-Boltzmann function fitting. (C) Data showing the translational velocities vs. time for phagosome A and B as indicated. Dark lines are velocities values after wavelet denoising. 
A

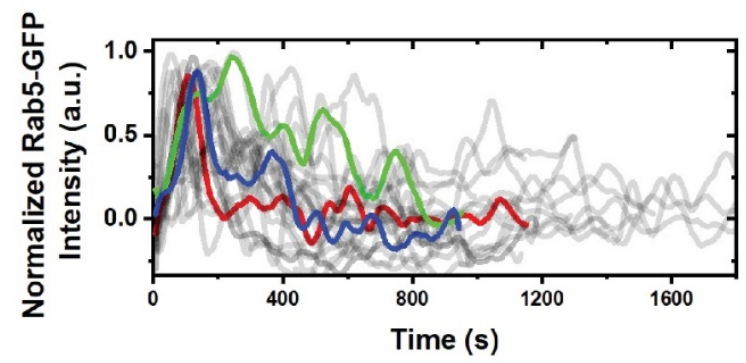

B

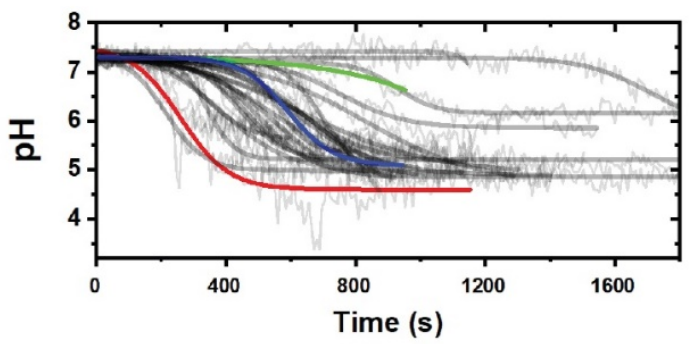

Figure 5-figure supplement 3. Plots showing normalized fluorescence intensity of Rab5-GFP (A) and phagosome $\mathrm{pH}(\mathbf{B})$ as a function of time. Each curve represents data of a single phagosome. In (A) and (B), data of three representative phagosomes are highlighted in red, blue, and green. In (B), thick lines are sigmoidal-Boltzmann function fitting to the individual phagosome acidification profile. 
A

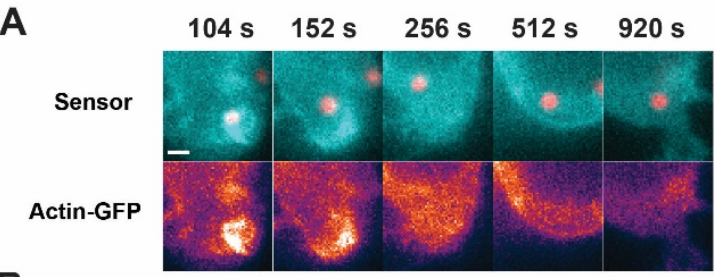

B

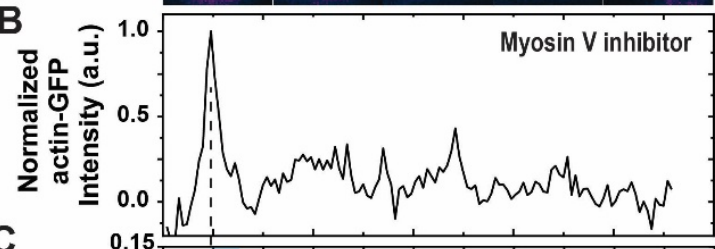

C

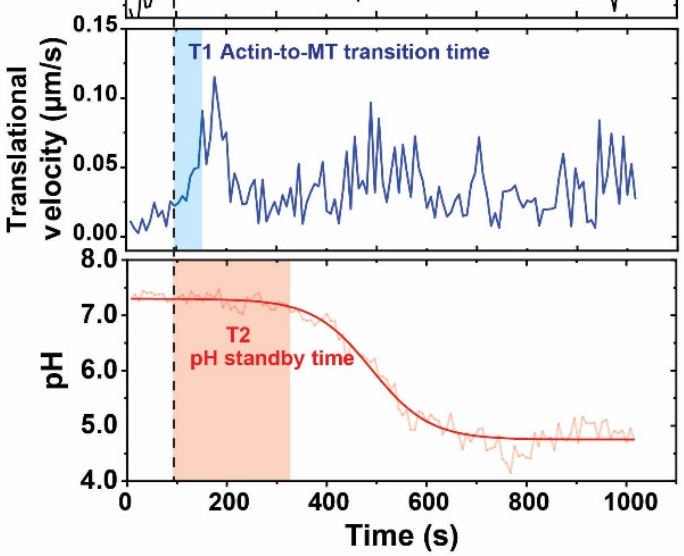

E

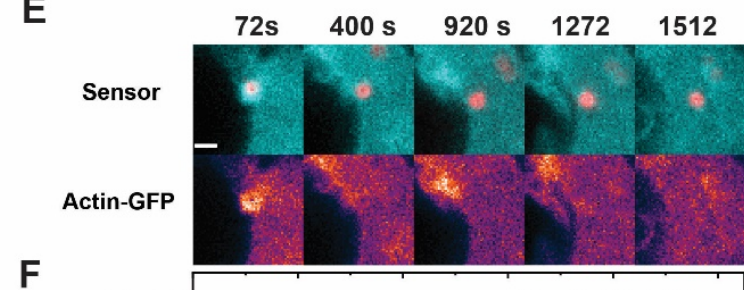

$\mathbf{F}$

G

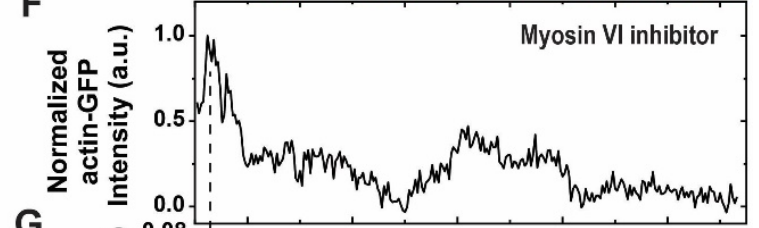

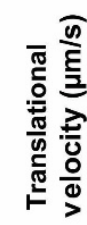

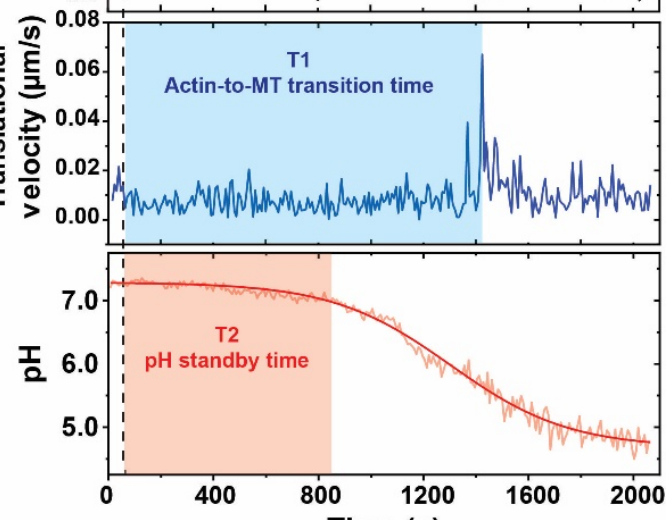

Figure 5-figure supplement 4. Phagosome dynamics and acidification after Myosin $\mathrm{V}(\mathbf{A}-\mathbf{D})$ and Myosin VI inhibition (E-H). (A and E) Fluorescence images showing subcellular locations of two single phagosomes at various time points in actin-GFP expressing macrophage cells. Scale bar, 2 $\mu \mathrm{m}$. (B and F) Plots showing normalized fluorescence intensity of actin-GFP around the phagosome of interest as a function of time. (C and G) Translational velocities of the two phagosomes of interest are plotted as a function of time. The blue shades indicate the actin-tomicrotubule transition period ( $\mathrm{T}_{1}$ ). ( $\mathbf{D}$ and $\mathbf{H}$ ) Plots showing $\mathrm{pH}$ vs. time for the two phagosomes of interest. The red shades indicate $\mathrm{pH}$ standby time $\left(\mathrm{T}_{2}\right)$. 
A

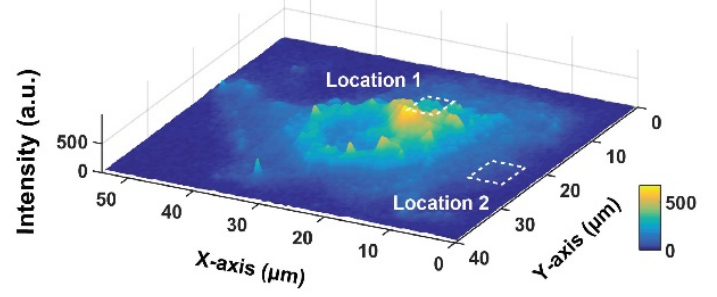

B
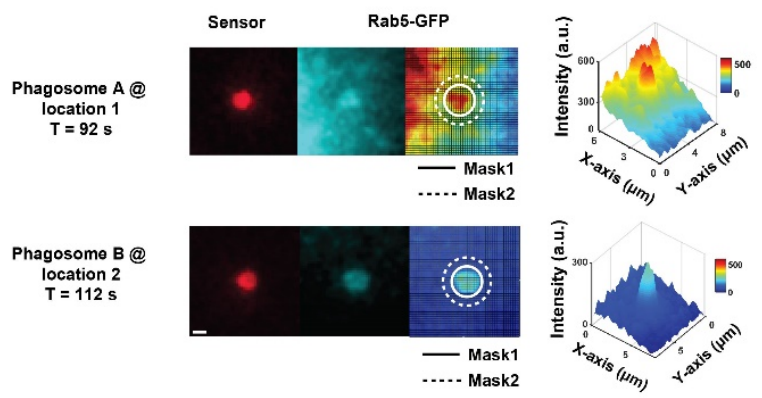

C

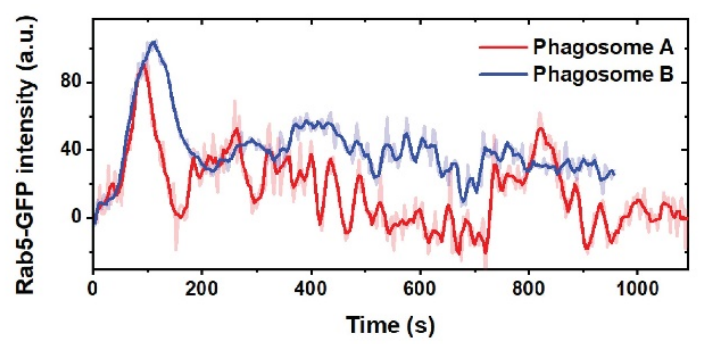

Figure 5-figure supplement 5. Recruitment of Rab5 on phagosomes differs with subcellular locations. (A) A representative 3D intensity histogram showing inhomogeneous fluorescence distribution of Rab5-GFP in an activated RAW 264.7 macrophage cell (in this case Rab5-GFP). (B) To quantify intensity of Rab5-GFP on single phagosomes and its cytosolic background, two circular masks, indicated as mask 1 and mask 2, were super-imposed on the localized phagosome. The average pixel intensity within circular mask 1 was obtained as signal, and that in between circular mask 1 and 2 was obtained as cytosolic background level. Scale bar, $1 \mu \mathrm{m}$. (C) Plots showing the Rab5-GFP intensity after background subtraction for the two phagosomes of interest shown in (A). 

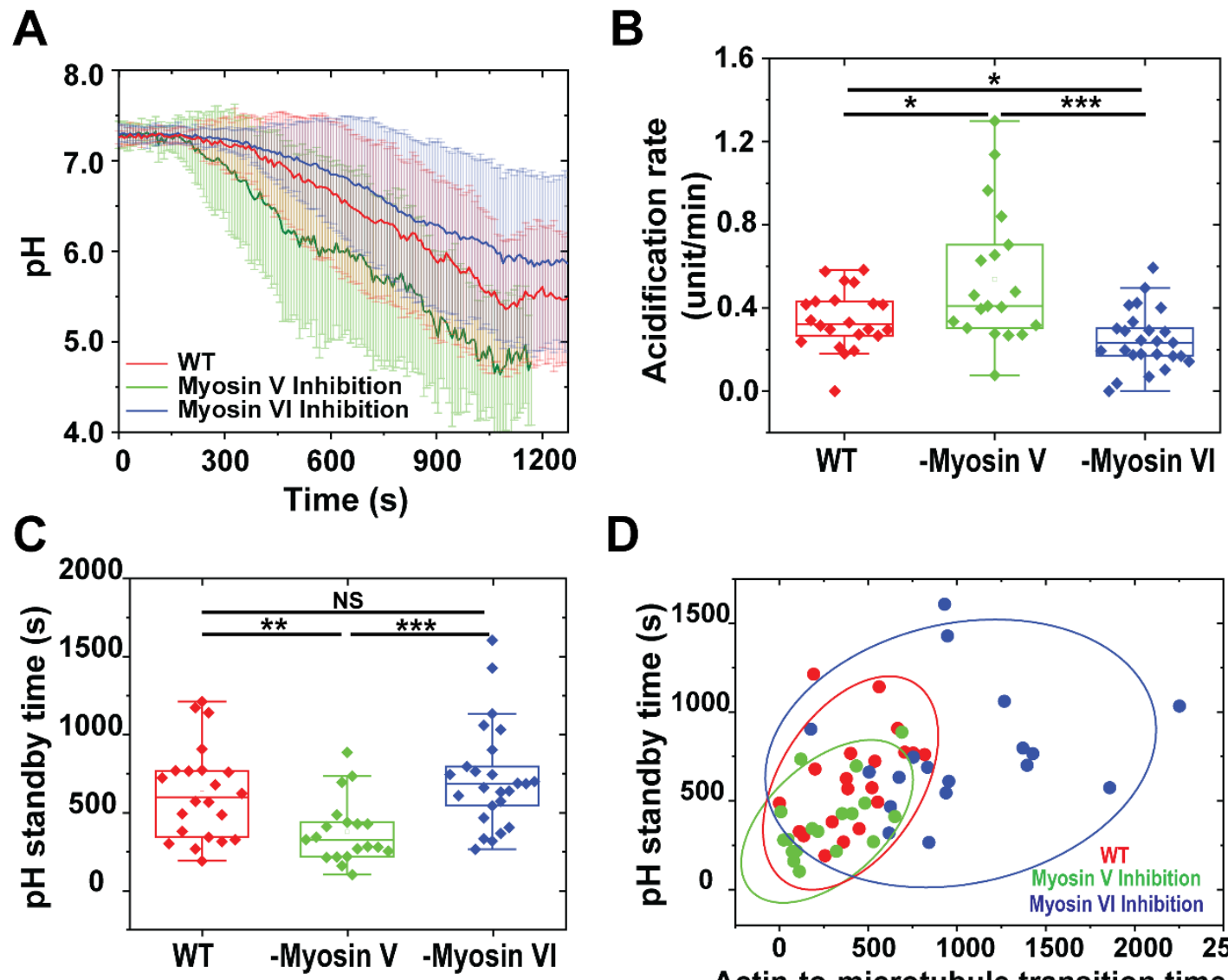

D

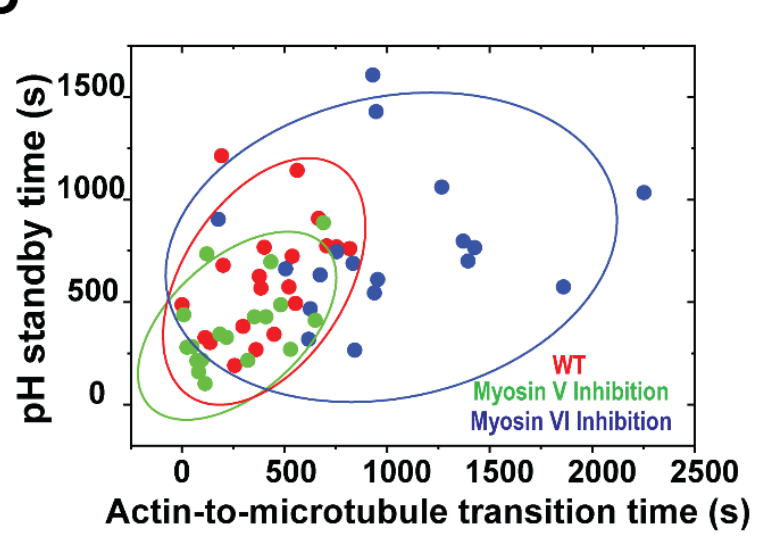

Figure 6. Dependence of phagosome acidification on actin-to-microtubule transport. (A) Line plots showing the average normalized $\mathrm{pH}$ as a function of time with or without drug treatments as indicated. The line curves are averaged from 22, 19, and 25 individual phagosomes in cells without drug treatment (wild type), with Myosin V inhibitor, and with Myosin VI inhibitor, respectively. Vertical bars represent standard deviations. (B) Scatter plot showing $\mathrm{pH}$ standby time of single phagosomes with or without drug treatments as indicated. The average $\mathrm{pH}$ standby time of phagosomes is $620 \pm 292 \mathrm{~s}$ without drug treatment (WT), $371 \pm 200 \mathrm{~s}$ with Myosin V inhibition, and $718 \pm 323 \mathrm{~s}$ with Myosin VI inhibition. (C) Scatter plot showing acidification rate of single phagosomes with or without drug treatments as indicated. The average acidification rate of phagosomes is $0.34 \pm 0.14 \mathrm{pH}$ unit/min in cells without drug treatment (WT), $0.54 \pm 0.32 \mathrm{pH}$ unit/min with Myosin V inhibition, and $0.25 \pm 0.14 \mathrm{pH}$ unit/min with Myosin VI inhibition. In all scatter plots, each box plot indicates the mean (horizontal line) and the interquartile range from $25 \%$ to $75 \%$ of the corresponding data set. Statistical significance is highlighted by $p$-values (student's t-test) as follows: ${ }^{*} p<0.05,{ }^{* *} p<0.01,{ }^{* *} p<0.001$, NS $p>0.05$. (D) Scatter plot showing $p H$ standby time against actin-to-microtubule transition time of single phagosomes with or without drug treatments as indicated. 


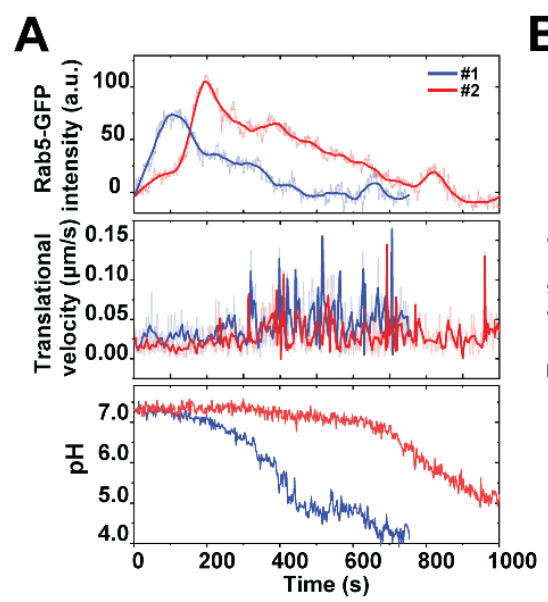

B

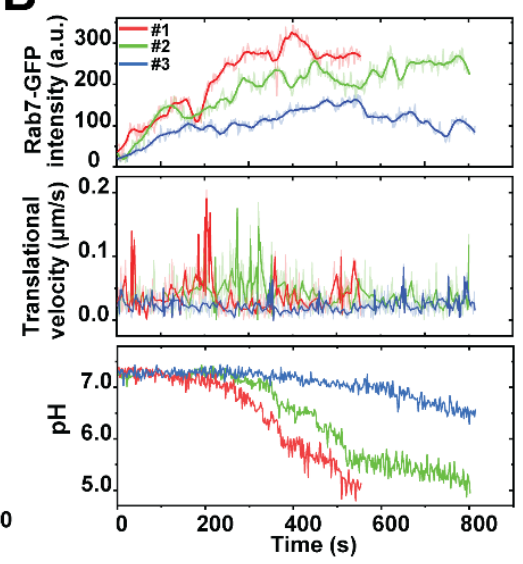

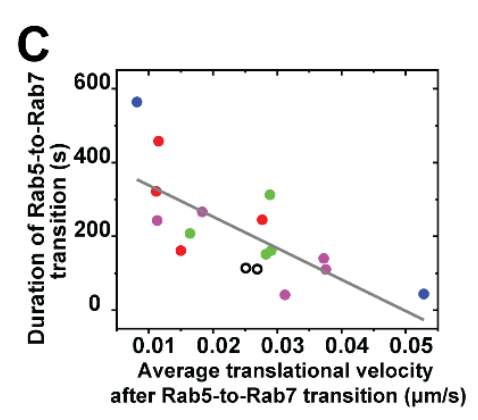

Figure 7. Rab5 and Rab7 recruitment and transport dynamics of single phagosomes from same cells. (A) Fluorescence intensity of Rab5-GFP, translational velocity, and phagosome lumen $\mathrm{pH}$ from three individual phagosomes within a same cell are plotted as a function of time. (B) Fluorescence intensity of Rab7-GFP, translational velocity, and phagosome lumen $\mathrm{pH}$ from three individual phagosomes within a same cell are plotted as a function of time. (C) Scatter plot showing the duration of Rab5-to-Rab7 conversion of individual phagosomes against their translational velocity after the Rab5-to-Rab7 conversion phase in activated RAW264.7 macrophage cells. Data points from multiple phagosomes within the same cells are shown in the same solid color. Data points from cells containing only one phagosome are shown as black circles. The linear regression has a Pearson's coefficient of -0.72 . 